\author{
Universidade de São Paulo \\ Instituto de Física
}

\title{
Estabilidade de Modos Quasinormais e uma Possível Interpretação na Correspondência AdS/CFT
}

\author{
Carlos Eduardo Pellicer de Oliveira
}

Orientador: Prof. Dr. Elcio Abdalla

Tese de doutorado apresentada ao Instituto de Física para a obtenção do título de Doutor em Ciências

\section{Comissão examinadora:}

Prof. Dr. Elcio Abdalla (IFUSP)

Prof. Dr. Paulo Teotônio Sobrinho (IFUSP)

Prof. Dr. Carlos Molina Mendes (IFUSP)

Prof. Dr. George Emanuel Avraam Matsas (IFT/UNESP)

Prof. Dr. Jorge Ernesto Hovarth (IAG/USP)

São Paulo

2011 


\section{FICHA CATALOGRÁFICA}

Preparada pelo Serviço de Biblioteca e Informação do Instituto de Física da Universidade de São Paulo

Oliveira, Carlos Eduardo Pellicer de

Estabilidade de modos quasinormais e uma possível interpretação na correspondência AdS/CFT.

São Paulo, 2011

Tese (Doutorado) - Universidade de São Paulo.

Instituto de Física, Departamento de Física Matemática

Orientador: Prof. Dr. Elcio Abdalla

Área de Concentração: Física

Unitermos: 1. Gravitação; 2. Buracos Negros;

3. Modos Quasinormais; 4. Correspondência AdS/CFT.

USP/IF/SBI-087/2011 
"Dedico este trabalho a todos que me ajudaram e me apoiaram nesta etapa da minha vida" 



\section{Agradecimentos}

À toda a minha família, em especial meus pais, meus irmãos e minhas sobrinhas.

Ao meu orientador Prof. Elcio Abdalla, por todo o trabalho que desenvolvemos juntos.

Aos colegas de sala e de departamento, com quem sempre pude contar em todas as dúvidas que tive no meu trabalho.

Às secretárias do departamento e ao pessoal do setor de informática, que me ajudaram em vários problemas não acadêmicos.

Aos meus amigos da graduação, que me acompanharam por vários anos e continuam sendo ótimas companhias.

Ao Sifu e a todos os instrutores e colegas da academia de Kung Fu, cujas lições de dedicação, perseverança e disciplina foram muito úteis neste trabalho.

À FAPESP, pelo apoio financeiro. 



\section{Resumo}

Esta tese é um estudo de estabilidade de modos quasinormais em um sistema que apresenta uma mudança de estabilidade ao variar continuamente os parâmetros físicos de um buraco negro. A mudança de estabilidade encontrada possui interpretações na correspondência AdS/CFT.

A ferramenta principal utilizada neste trabalho para o cálculo de modos quasinormais foram métodos numéricos que podem ser utilizados em inúmeros trabalhos desta área de pesquisa, especialmente por não dependerem de suposições de simetria ou de comportamento conveniente do sistema físico. 



\section{Abstract}

This thesis is a study of stability of quasinormal modes in a system featuring a stability change if one continuously varies the physical parameters of a black hole. The stability change thus found has some possible interpretations in the AdS/CFT correspondence.

The main tool used in this study for calculating quasinormal modes are numerical methods that can be used in numerous works in this area of research, especially because they do not depend on assumptions of symmetry or any convenient behavior of the physical system. 



\section{Sumário}

Agradecimentos $\quad$ v

Resumo vii

$\begin{array}{ll}\text { Abstract } & \text { ix }\end{array}$

Notações e convenções $\quad$ Xv

1 Introdução 1

2 Modos Quasinormais $\quad 7$

2.1 Definição de modos normais e quasinormais . . . . . . . . . . . 7

2.2 Equações de perturbação . . . . . . . . . . . . . . . . . . . . . 8

2.3 Estabilidade dos modos quasinormais . . . . . . . . . . 11

2.4 Método WKB . . . . . . . . . . . . . . . . . . . . . . . 14

2.5 Método de Horowitz-Hubeny . . . . . . . . . . . . . . . . . . . . . . . . . .

2.6 Integração numérica . . . . . . . . . . . . . . . . 16

3 Correspondência AdS/CFT 19

3.1 Motivações e origens da correspondência . . . . . . . . . . . . . 19

3.2 Princípio holográfico . . . . . . . . . . . . . . . . . 20

3.3 Alguns "verbetes" do dicionário AdS/CFT . . . . . . . . . . . 21

3.4 Supercondutores holográficos . . . . . . . . . . . . . . 23

3.5 Papel dos modos quasinormais na correspondência AdS/CFT . . . 26

4 Transições de fase 29

4.1 Modos marginalmente estáveis . . . . . . . . . . . . . . 30

4.2 Modos Quasinormais . . . . . . . . . . . . . . . 35

4.3 Transições de fase . . . . . . . . . . . . . . . . . . 39

5 Discussões finais $\quad 51$ 
A Métodos numéricos $\quad 53$

A.1 Método de busca de soluções . . . . . . . . . . . . . . . 53

A.1.1 Método da dicotomia . . . . . . . . . . . . . 53

A.1.2 Método de Newton-Raphson . . . . . . . . . . . . 54

A.1.3 Método de busca por gradientes . . . . . . . . . . . 56

A.1.4 Raízes de polinômios . . . . . . . . . . . . . . 56

A.1.5 Método de Laguerre . . . . . . . . . . . . . . 57

A.1.6 Método de Jenkins-Traub . . . . . . . . . . . . . . 59

A.1.7 Polinômios mal condicionados . . . . . . . . . . . 63

A.2 Integração de funções . . . . . . . . . . . . . . . . . . . . 64

A.2.1 Método do ponto intermediário . . . . . . . . . . 64

A.2.2 Método do Trapézio . . . . . . . . . . . . . . 65

A.2.3 Regra de Simpson . . . . . . . . . . . . . 66

A.3 Equações Diferenciais Ordinárias . . . . . . . . . . . . . . 68

A.3.1 Método de Euler . . . . . . . . . . . . . . . . 68

A.3.2 Método de Runge-Kutta . . . . . . . . . . . . . . . 68

A.4 Equações Diferenciais Parciais . . . . . . . . . . . . . . . . 71

A.4.1 Método de Diferenças Finitas . . . . . . . . . . . 72

A.4.2 Equações hiperbólicas . . . . . . . . . . . . . 74

A.4.3 Estabilidade de von Neumann . . . . . . . . . . . . . 77

A.4.4 Equações parabólicas . . . . . . . . . . . . . . . . . . 77

A.4.5 Equações elípticas . . . . . . . . . . . . . 78

B Código numérico do método de diferanças finitas. 81

C Código numérico do método de Horowitz-Hubeny. 87

$\begin{array}{ll}\text { Referências Bibliográficas } & 101\end{array}$ 


\section{Lista de Figuras}

2.1 Exemplo de potenciais de Regge-Wheeler e Zerilli com $M=1 \ldots$. . . . 14

4.1 Modos reproduzidos e um modo adicional para $k=1$ e $m^{2}>0$. . 32

4.2 Modos previstos para $k=0$ e $m^{2}>0 \ldots \ldots 33$

4.33 modos marginalmente estáveis encontrados, seguido de um decaimento de lei de potência. . . . . . . . . . . . . . . . . 34

4.4 Modos marginalmente estáveis encontrados como previsto. . . . . 35

4.5 Gráfico da coordenada tartaruga com $k=1, L=1, Q=1$ e $r_{H}=1 . \quad . \quad$. 37

4.6 Potenciais efetivos em função de $r$ para diferentes valores de $L$. . . . . . 40

4.7 Potenciais efetivos em função de $r$ para diferentes valores de $L$. . . . . . 41

4.8 Modos quasinormais em função de $t$. . . . . . . . . . . . . . . . . . . 42

4.9 Frequências do modo principal. . . . . . . . . . . . . . . . 43

4.10 Frequências do modo principal próximas à mudança de estabilidade. . . 44

4.11 Comportamento do sistema e do segundo modo em função de $t$. . . . . 44

4.12 Comportamento do segundo modo em função de $t$. . . . . . . . . . . 45

4.13 Frequências do segundo modo. . . . . . . . . . . . . . . . . 45

4.14 Comparação dos valores da parte real da frequência obtidos por integração numérica e pelo método de Horowitz-Hubeny. . . . . . . . . . . 46

4.15 Comparação dos valores da parte imaginária de frequência obtidos por integração numérica e pelo método de Horowitz-Hubeny. . . . . . . . . 46

4.16 Frequências do modo principal em função da temperatura. . . . . . . . 47

4.17 Frequências do modo principal em função da temperatura próximas à mudança de estabilidade. . . . . . . . . . . . . . . . . 47

4.18 Frequências do segundo modo em função da temperatura. . . . . . . . 48

4.19 Modos quasinormais estáveis e instáveis. . . . . . . . . . . . 48

4.20 Modos quasinormais estáveis e instáveis. . . . . . . . . . . . . 49

4.21 Modos quasinormais estáveis e instáveis. . . . . . . . . . . . . . . . . 49

4.22 Modos quasinormais estáveis e instáveis. . . . . . . . . . . . . 50

4.23 Modos quasinormais estáveis e instáveis. . . . . . . . . . . . 50

A.1 Passos do método de Newton. . . . . . . . . . . . . . . 55 
A.2 Aproximação dada pelo método do ponto intermediário. . . . . . . . . 65

A.3 Aproximação dada pelo método do trapézio. . . . . . . . . . . . . 66

A.4 Aproximação dada pela regra de Simpson. . . . . . . . . . . . . . 67

A.5 Células computacionais para $\frac{\partial f}{\partial x}(x, y) \mathrm{e} \frac{\partial^{2} f}{\partial x^{2}}(x, y) \ldots \ldots \ldots \ldots$. . . . . . . . . . . . . . . . . . . . .

A.6 Células computacionais para $\frac{\partial f}{\partial y}(x, y)$ e $\frac{\partial^{2} f}{\partial y^{2}}(x, y) \ldots \ldots \ldots 75$

A.7 Células computacionais para $\frac{\partial^{2} f}{\partial x \partial y}(x, y)$ e $\nabla^{2} f(x, y) \ldots \ldots \ldots 75$

A.8 Domínio de integração de uma equação hiperbólica. . . . . . . . . . 76

A.9 Domínio de integração de uma equação elíptica. . . . . . . . . . . . . 79

A.10 Ordem de relaxação. . . . . . . . . . . . . . . . . . 80 


\section{Notações e convenções}

- Índices latinos (como $i, j$ e $k$ ) rotulam as coordenadas espaciais e variam de 1 a $d-1$, onde $d$ é o número de dimensões. Índices gregos (como $\mu, v$ e $\sigma$ ) rotulam as coordenadas do espaço-tempo, variando de 0 a $d-1$, sendo 0 o rótulo da coordenada temporal.

- A métrica de um espaço-tempo plano com 4 dimensões em coordenadas cartesianas tem os elementos diagonais dados por $(-1,+1,+1,+1)$.

- Índices repetidos denotam uma soma sobre os valores que estes índices podem assumir.

- Derivadas parciais na direção radial são denotadas com uma linha sobrescrita ('), enquanto derivadas no tempo são denotadas com um ponto diacrítico $\left({ }^{*}\right)$.

- Exceto quando são denotadas explicitamente, a velocidade da luz $c$, a constante da gravitação $G$, a constante de Planck $\hbar$ e a constante de Boltzmann são tomadas como $c=\hbar=G=k_{\mathrm{B}}=\frac{1}{4 \pi \varepsilon_{0}}=1$. 



\section{Capítulo 1}

\section{Introdução}

A Relatividade Geral é uma teoria desenvolvida por Albert Einstein em 1915 (referência [1]) que descreve como uma distribuição de matéria deforma o espaçotempo em sua volta. Matematicamente a forma do espaço-tempo é dada por uma métrica,

$$
\mathrm{d} s^{2}=g_{\mu v} \mathrm{~d} x^{\mu} \mathrm{d} x^{\nu}
$$

que mede a distância infinitesimal entre dois pontos. Em um espaço euclidiano, a equação acima é o teorema de Pitágoras.

Uma analogia interessante é entender o espaço-tempo como um lençol esticado com uma bola de boliche no meio do lençol. Com o peso da bola de boliche, a superfície do lençol deixa de ser plana e passa a ser descrita por uma superfície em duas dimensões imersa em um espaço de três dimensões. A distância infinitesimal entre dois pontos do lençol pode ser dada como uma distância euclidiana em três dimensões ou por uma métrica induzida no lençol, dada pela deformação causada pela bola de boliche. Um observador olhando o lençol de fora (i.e., localizado no espaço euclidiano de três dimensões) observa bolinhas de gude indo em direção à bola de boliche (ou orbitando) devido à geometria do lençol, enquanto um observador que só mede distância no lençol observa uma força de atração entre a bola de boliche e a bolinha de gude.

A Relatividade Geral parte de uma série de suposições. Uma delas é que a interação gravitacional se deve à geometria do espaço-tempo, descrita por uma métrica. Outra é que a matéria é fonte de gravidade, e as equações que relacionam a métrica do espaço-tempo com a distribuição de matéria são chamadas de equações de Einstein, expressa por

$$
R_{\mu v}-\frac{1}{2} R g_{\mu v}=8 \pi T_{\mu v} .
$$

$T_{\mu \nu}$ é o tensor de energia-momento. 
Estas equações podem ser obtidas por um princípio variacional a partir da ação de Einstein-Hilbert

$$
S_{\mathrm{EH}}=\frac{1}{16 \pi G} \int \mathrm{d}^{4} x \sqrt{-g} R .
$$

A ação (1.3) sozinha implica em

$$
R_{\mu v}-\frac{1}{2} R g_{\mu v}=0
$$

Para obter o lado direito da equação (1.2) é preciso acoplar a ação de EinsteinHilbert com uma ação da matéria, que de um modo geral é dada por

$$
S_{\mathrm{M}}=-\frac{1}{2} \int \mathrm{d}^{4} x \sqrt{-g} \nabla_{\mu} \phi \nabla^{\mu} \phi
$$

Definindo

$$
T_{\mu \nu}=-\frac{2}{\sqrt{-g}} \frac{\delta S_{\mathrm{M}}}{\delta g^{\mu \nu}},
$$

as equações de movimento para $g_{\mu \nu}$ obtidas da ação $S_{\mathrm{EH}}+S_{\mathrm{M}}$ são expressas por (1.2).

As equações de Einstein no vácuo (1.4) admitem uma solução encontrada por Schwarzschild em 1916 [2], supondo uma métrica com simetria esférica e assintoticamente plana. A solução é dada por

$$
\mathrm{d} s^{2}=-f(r) \mathrm{d} t^{2}+\frac{1}{f(r)} \mathrm{d} r^{2}+r^{2} \mathrm{~d} \Omega^{2}
$$

onde $f(r)=1-\frac{2 M}{r}$ e $\mathrm{d} \Omega^{2}=\mathrm{d} \theta^{2}+\operatorname{sen}^{2} \theta \mathrm{d} \varphi^{2}$ é a métrica de uma esfera $S_{2}$ de raio unitário. A constante $M$ aparece como uma constante de integração permitida, que é identificada como a massa de um corpo localizado em $r=0$ no limite newtoniano.

A métrica de Schwarzschild é singular em $r=0$ e em $r=2 M$, mas esta singularidade pode ser removida com uma transformação de coordenadas conhecida como

$$
\tilde{t}=t+2 M \log \left(\frac{r}{2 M}-1\right)
$$

com a qual podemos escrever a métrica como

$$
\mathrm{d} s^{2}=-\left(1-\frac{2 M}{r}\right) \mathrm{d} \tilde{t}^{2}+\frac{4 M}{r} \mathrm{~d} \tilde{t} \mathrm{~d} r+\left(1+\frac{2 M}{r}\right) \mathrm{d} r^{2}+r^{2} \mathrm{~d} \Omega^{2} .
$$

Estas coordenadas são chamadas de coordenadas de Eddington-Finkelstein. 
Outra forma conveniente de escrever a métrica de Schwarzschild é dada por Kruskal em [3]. Seja a coordenada tartaruga definida por $\mathrm{d} x=\frac{\mathrm{d} r}{f(r)}$ cuja solução é dada por

$$
x(r)=r+2 M \log \left(\frac{r}{2 M}-1\right) .
$$

Nesta coordenada, a métrica é escrita como

$$
\mathrm{d} s^{2}=\left(1-\frac{2 M}{r(x)}\right)\left(-\mathrm{d} t^{2}+\mathrm{d} x^{2}\right)+r^{2}(x) \mathrm{d} \Omega^{2} .
$$

Analisando a estrutura do espaço-tempo, ${ }^{1}$ podemos ver que uma partícula viajando a uma velocidade menor ou igual à velocidade da luz pode ir de uma região com $r>2 M$ para uma região com $r<2 M$, mas não o contrário. Um observador situado em $r>2 M$ não pode obter informações vindas de $r<2 M,^{2}$ por este motivo, a superfície $r=2 M$ é chamada de horizonte de eventos do buraco negro.

Uma segunda solução de interesse das equações de Einstein descreve um buraco negro com carga elétrica. Dado o tensor de energia-momento de um campo elétrico radial, obtemos uma solução para a métrica na forma da equação (1.7), como pode ser visto em $[5 ; 6]$. No limite newtoniano, a força sentida por um observador deve ser a mesma prevista pela lei de Gauß. A dedução da solução de Reissner-Nordström é feita com detalhes em [7] e a solução é dada por uma métrica esfericamente simétrica com

$$
f(r)=1-\frac{2 M}{r}+\frac{Q^{2}}{4 r^{2}}
$$

Assim como na métrica de Schwarzschild, a solução de Reissner-Nordström apresenta singularidades aparentes em $r_{ \pm}=M \pm \sqrt{M^{2}-\frac{Q^{2}}{4}}$. A análise do espaço tempo mostra que $r_{+}$é o horizonte de eventos se for um valor real. Se $Q^{2}>4 M^{2}$, a singularidade presente em $r=0$ não está "coberta" por um horizonte de eventos e é dita "nua". O princípio da censura cósmica exclui tais soluções.

A próxima solução de interesse a ser tratada é o espaço AdS (Anti de Sitter), que é um espaço de assinatura lorentziana com curvatura constante e negativa. $\mathrm{O}$ espaço AdS pode ser definido pela superfície $d$-dimensional imersa em um espaço $(d+1)$-dimensional

$$
-x_{0}^{2}+\sum_{i=1}^{d-1} x_{i}^{2}-x_{d}^{2}=-L^{2}
$$

\footnotetext{
${ }^{1}$ Esta análise é feito com rigor por diagramas de penrose e pode ser vista em [4].

${ }^{2}$ Classicamente. Efeitos quânticos como radiação Hawking violam esta afirmação.
} 
que é invariante por transformações pertencentes ao grupo $\mathrm{SO}(2, d-1)$. A métrica induzida do espaço AdS escrita em coordenadas de Poincaré é

$$
\mathrm{d} s^{2}=\frac{L^{2}}{z^{2}}\left(-\mathrm{d} t^{2}+\sum_{i=1}^{d-2} \mathrm{~d} x_{i}^{2}+\mathrm{d} z^{2}\right)
$$

onde $z \in(0, \infty)$ e as outras coordenadas podem assumir qualquer valor real.

O espaço AdS é solução das equações de Einstein se à lagrangiana de EinsteinHilbert for somada um termo constante conhecido como constante cosmológica,

$$
S=\frac{1}{16 \pi} \int \mathrm{d}^{4} x \sqrt{-g}(R+2 \Lambda)
$$

de modo que as equações de movimento são dadas por

$$
R_{\mu v}-\frac{1}{2} R g_{\mu v}+\Lambda g_{\mu v}=0
$$

A constante $\Lambda$ deve ser negativa para que o espaço AdS seja solução da equação (1.16). A solução, em 4 dimensões com simetria esférica, pode ser escrita como

$$
\mathrm{d} s^{2}=-\left(1+\frac{r^{2}}{L^{2}}\right) \mathrm{d} t^{2}+\frac{1}{\left(1+\frac{r^{2}}{L^{2}}\right)} \mathrm{d} r^{2}+r^{2} \mathrm{~d} \Omega^{2},
$$

onde $L^{2}=-\frac{3}{\Lambda}$. Um buraco negro esfericamente simétrico imerso em um espaço AdS também possui soluções dadas por

$$
\mathrm{d} s^{2}=-f(r) \mathrm{d} t^{2}+\frac{1}{f(r)} \mathrm{d} r^{2}+r^{2} \mathrm{~d} \Omega^{2}
$$

com a diferença que $f(r)$ não é mais assintoticamente plano, deve ser proporcional a $1+\frac{r^{2}}{L^{2}}$. As soluções de interesse são Schwarzschild-Anti de Sitter com

$$
f(r)=1-\frac{2 M}{r}+\frac{r^{2}}{L^{2}}
$$

e Reissner-Nordström-Anti de Sitter com

$$
f(r)=1-\frac{2 M}{r}+\frac{Q^{2}}{4 r^{2}}+\frac{r^{2}}{L^{2}} .
$$

Neste trabalho estudamos a estabilidade de perturbações escalares em um espaço-tempo Reisser Nordström assintoticamente AdS. Esta estabilidade é estudada calculando modos quasinormais. No capítulo 2 explicamos o que são os 
modos quasinormais, que descrevem o comportamento de perturbações de soluções conhecidas das equações de Einstein. Descrevemos critérios de estabilidade dos modos quasinormais e alguns métodos conhecidos para calcular os modos. Além de nos dizer se o espaço-tempo em questão é estável, como os modos quasinormais dependem dos parâmetros físicos do buraco negro, uma medida de modos quasinormais (através de ondas gravitacionais, por exemplo) pode nos dar informações da massa, carga e velocidade angular do buraco negro.

O interesse em modos quasinormais em espaços-tempos assintoticamente AdS vem da Correspondência AdS/CFT, que relaciona modos quasinormais com estados térmicos em uma teoria de campo conforme. A motivação do estudo desta correspondência vem do fato de que podemos utilizá-la para entender teorias de campos conformalmente invariantes mapeando-as em uma teoria de gravitação clássica. No capítulo 3 mencionamos alguns tópicos de interesse da Correspondência AdS/CFT, em especial o conceito de supercondutores holográficos e o papel dos modos quasinormais na correspondência.

No capítulo 4 detalhamos o trabalho publicado em [8]. Em especial, detalhamos como utilizamos métodos numéricos (listados no apêndice A) para calcular modos quasinormais de perturbações com massa e carga elétrica em um espaçotempo Reissner-Nordström-anti de Sitter. Comparamos a estabilidade dos modos quasinormais encontrados com propriedades dos supercondutores holográficos, em especial verificamos que a fase que mostra um valor diferente de zero para um parâmetro de ordem que descreve uma transição de fase está associada a instabilidades dos modos quasinormais, enquanto a fase com parâmetro de ordem igual a zero está associada a modos quasinormais estáveis. 



\section{Capítulo 2}

\section{Modos Quasinormais}

Quando batemos em um diapasão contra uma superfície, ouvimos um som com uma frequência específica (na maioria dos diapasões, é um Lá de $440 \mathrm{~Hz}$ ). Analisando cientificamente este fenômeno, vemos que o diapasão, quando perturbado (i.e. a sua configuração padrão é alterada), vibra com uma frequência $\omega$ que depende das propriedades físicas do diapasão cuja intensidade diminui com o tempo, de acordo com uma exponencial $e^{-\delta t}$. O ar em volta do diapasão vibra na mesma frequência, propagando o som. A propagação pode ser descrita por uma função proporcional a $e^{i \omega t}$, onde $\omega$ é um número complexo, cuja parte real representa a frequência do som e cuja parte imaginária representa o decaimento do som.

Analogamente, um buraco negro com uma certa configuração, sob o efeito de uma perturbação, gera uma vibração no espaço-tempo deformado pelo buraco negro. Esta vibração, assim como as ondas sonoras emitidas por um diapasão, é descrita por uma função proporcional a $e^{i \omega t}$, com $\omega$ complexo. Na analogia feita no capítulo 1, a perturbação seria um golpe na bola de boliche, que ao vibrar, gera uma vibração no lençol deformado pelo peso da bola. Essa vibração do espaçotempo é entendida como um modo quasinormal. Por esta analogia, Nollert, em [9] chama os modos quasinormais de "sons" característicos de buracos negros.

\subsection{Definição de modos normais e quasinormais}

Um sistema físico regular sem dissipação de energia pode ser descrito por um conjunto de funções que obedecem a uma certa condição de contorno. A condição inicial do sistema determina qual superposição destas funções descreve a configuração do sistema, que se comporta como uma oscilação com uma frequência específica para cada função mencionada. As funções que obedecem às condições de contorno são chamadas de modos normais. 
Em um sistema dissipativo, o comportamento do sistema também é descrito por uma superposição de modos que atendem a uma condição de contorno e que oscilam com uma frequência específica. As oscilações, no entanto, são amortecidas e, como já mencionado, são proporcionais a $e^{i \omega t}$ com $\omega$ complexo.

Kokkotas e Schmidt definem em [10] modos quasinormais de uma maneira mais formal. Seja a equação de onda unidimensional

$$
-\frac{\partial^{2} u}{\partial t^{2}}+\frac{\partial^{2} u}{\partial x^{2}}-V(x) u=0
$$

com $u(x, 0)=f(x)$ e $\frac{\partial u}{\partial t}(x, 0)=g(x)$. A transformada de Laplace $\tilde{u}(x, s)$ é tal que

$$
s^{2} \tilde{u}(x, s)-\frac{\partial^{2} \tilde{u}}{\partial x^{2}}(x, s)+V(x) \tilde{u}(x, s)=s f(x)+g(x) .
$$

Sejam $\tilde{u}_{+}(x, s)$ e $\tilde{u}_{-}(x, s)$ as soluções homogêneas da equação (2.2), definimos a função de Green como

$$
G\left(x, x^{\prime}, s\right)=\frac{1}{W(s)} \begin{cases}\tilde{u}_{-}\left(x^{\prime}, s\right) \tilde{u}_{+}(x, s) & \text { se } x^{\prime}<x \\ \tilde{u}_{-}(x, s) \tilde{u}_{+}\left(x^{\prime}, s\right) & \text { se } x^{\prime}>x\end{cases}
$$

onde $W(s)$ é o Wronskiano das soluções homogêneas. A solução geral é dada por

$$
\tilde{u}(x, s)=\int_{-\infty}^{+\infty} G\left(x, x^{\prime}, s\right)\left[s f\left(x^{\prime}\right)+g\left(x^{\prime}\right)\right] \mathrm{d} x^{\prime} .
$$

Seja um valor de $s$ para o qual as soluções homogêneas $\tilde{u}_{+}$e $\tilde{u}_{-}$são linearmentes dependentes, o que torna o Wronskiano nulo e a função de Green (2.3) singular. Os valores de $s$ que obedecem a

$$
\tilde{u}_{+}(x, s)=\alpha(s) \tilde{u}_{-}(x, s)
$$

são definidos como frequências quasinormais em [10].

\subsection{Equações de perturbação}

Regge e Wheeler publicaram em [11] um dos primeiros trabalhos em perturbações de buracos negros. Supondo uma métrica de fundo conhecida, como por exemplo as métricas de buracos negros vistas no capítulo 1 , denotada por $\stackrel{0}{g}_{\mu v}$. A esta métrica acrescentamos uma perturbação, denotada por $h_{\mu v}$, cujos termos devem ser pequenos se comparados aos termos da métrica de fundo. A métrica total do sistema é dada por

$$
g_{\mu v}=\stackrel{0}{g}_{\mu v}+h_{\mu v}
$$


Sejam

$$
\begin{aligned}
& \Gamma_{\mu v}^{\lambda}=\stackrel{0}{\Gamma}_{\mu v}^{\lambda}+\delta \Gamma_{\mu v}^{\lambda}, \\
& R_{\mu v}={ }^{0} R_{\mu v}+\delta R_{\mu v},
\end{aligned}
$$

onde os termos $\stackrel{0}{\Gamma}_{\mu \nu}^{\lambda}$ e $\stackrel{0}{R}_{\mu v}$ são os valores de $\Gamma_{\mu \nu}^{\lambda}$ e $R_{\mu v}$ com $h_{\mu v}=0$ e os termos precedidos de $\delta$ possuem termos em até primeira ordem de $h_{\mu v}$, isto é, os termos com uma potência maior que um em $h_{\mu v}$ são desprezados.

Segue que

$$
\begin{aligned}
\delta \Gamma_{\mu v}^{\lambda} & =\frac{1}{2}{ }_{g}^{0} \lambda \alpha\left(h_{\alpha v, \mu}+h_{\alpha \mu, v}-h_{\mu v, \alpha}\right)-{ }_{g}^{0} \lambda \alpha{ }^{0} \Gamma_{\mu v}^{\beta} h_{\alpha \beta} \\
& =\frac{1}{2}{ }_{g}^{0} \lambda \alpha\left(h_{\alpha v ; \mu}+h_{\alpha \mu ; v}-h_{\mu v ; \alpha}\right), \\
\delta R_{\mu v} & =\delta \Gamma_{\mu \alpha, v}^{\alpha}-\delta \Gamma_{\mu v, \alpha}^{\alpha}+\stackrel{0}{\Gamma}_{v \beta}^{\alpha} \delta \Gamma_{v \alpha}^{\beta}+\stackrel{0}{\Gamma}_{\mu \alpha}^{\beta} \delta \Gamma_{v \beta}^{\alpha}-\stackrel{0}{\Gamma}_{\alpha \beta}^{\alpha} \delta \Gamma_{\mu v}^{\beta}-\stackrel{0}{\Gamma}_{\mu v}^{\beta} \delta \Gamma_{\alpha \beta}^{\alpha} \\
& =\delta \Gamma_{\mu \alpha ; v}^{\alpha}-\delta \Gamma_{\mu v ; \alpha}^{\alpha} .
\end{aligned}
$$

No vácuo, as equações de perturbação da métrica são escritas como

$$
\delta R_{\mu v}=0 .
$$

No trabalho de Regge e Wheeler [11], ou autores tratam de um buraco negro esfericamente simétrico. Aproveitando esta simetria, por uma rotação em torno da origem, os termos $h_{00}, h_{01}=h_{10}$ e $h_{11}$ se transformam como escalares, os pares $\left(h_{02}, h_{03}\right)=\left(h_{20}, h_{30}\right)$ e $\left(h_{12}, h_{13}\right)=\left(h_{21}, h_{31}\right)$ como vetores e os termos $h_{22}$, $h_{23}=h_{32}$ e $h_{33}$ como um tensor simétrico de quatro componentes. Para fazer uma separação de variáveis, denota-se os modos com paridade $(-1)^{l+1}$ como axiais, que podem ser escritos como

$$
h_{\mu v}=\left(\begin{array}{cccc}
0 & 0 & -h_{0} \frac{1}{\operatorname{sen} \theta} \frac{\partial}{\partial \varphi} Y_{l}^{m} & h_{0} \frac{1}{\operatorname{sen} \theta} \frac{\partial}{\partial \theta} Y_{l}^{m} \\
0 & 0 & -h_{1} \frac{1}{\operatorname{sen} \theta} \frac{\partial}{\partial \varphi} Y_{l}^{m} & h_{1} \frac{1}{\operatorname{sen} \theta} \frac{\partial}{\partial \theta} Y_{l}^{m} \\
* & * & h_{2} \cdot\left(\frac{1}{\operatorname{sen} \theta} \frac{\partial^{2}}{\partial \partial \partial \varphi}-\frac{\cos \theta}{\operatorname{sen} \theta} \frac{\partial}{\partial \phi}\right) Y_{l}^{m} & * \\
* & * & \frac{h_{2}}{2} \cdot\left(\frac{1}{\operatorname{sen} \theta} \frac{\partial^{2}}{\partial \varphi^{2}}+\cos \theta \frac{\partial}{\partial \theta}-\operatorname{sen} \theta \frac{\partial^{2}}{\partial \theta^{2}}\right) Y_{l}^{m} & -h_{2} \cdot\left(\operatorname{sen} \theta \frac{\partial^{2}}{\partial \theta \partial \varphi}-\cos \theta \frac{\partial}{\partial \varphi}\right) Y_{l}^{m}
\end{array}\right),
$$

onde um $*$ denota um termo simétrico a outro já expresso acima. As funções $h_{0}, h_{1}$ e $h_{2}$ dependem de $r$ e $t$, e os harmônicos esféricos $Y_{l}^{m}$ dependem de $\theta$ e $\varphi$.

Os termos com paridade $(-1)^{l}$, definidos como polares, são dados por

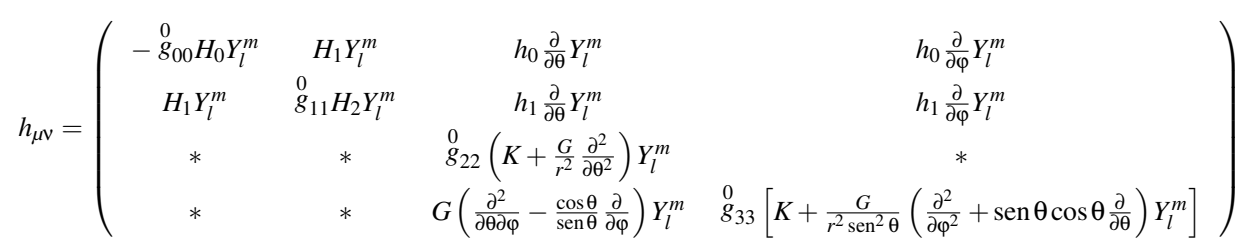


Assim como na equação (2.12), um $*$ denota um termo simétrico, as funções $H_{0}$, $H_{1}, h_{0}, h_{1}, G$ e $K$ dependem de $r$ e $t$ e os harmônicos esféricos $Y_{l}^{m}$ dependem de $\theta$ e $\varphi$.

Para simplificar as equações, Regge e Wheeler fazem uma transformação de calibre conveniente. Com a escolha de $m=0$, os modos polares são dados por

$$
h_{\mu \nu}=\left(\begin{array}{cccc}
0 & 0 & 0 & h_{0}(r, t) \\
0 & 0 & 0 & h_{1}(r, t) \\
0 & 0 & 0 & 0 \\
* & * & 0 & 0
\end{array}\right) \cdot \operatorname{sen} \theta \frac{\partial}{\partial \theta} P_{l}(\cos \theta)
$$

e os modos axiais

$$
h_{\mu v}=\left(\begin{array}{cccc}
-\stackrel{0}{g}_{00} H_{0}(r, t) & H_{1}(r, t) & 0 & 0 \\
H_{1}(r, t) & \stackrel{0}{g}_{11} H_{2}(r, t) & 0 & 0 \\
0 & 0 & \stackrel{0}{g}_{22} K(r, t) & 0 \\
0 & 0 & 0 & \stackrel{0}{g}_{33} K(r, t)
\end{array}\right) \cdot P_{l}(\cos \theta)
$$

Para os modos polares, tomando como métrica de fundo um buraco negro de Schwarzschild, as equações $\delta R_{\mu v}=0$, combinadas com a definição

$$
\psi(r, t)=\left(1-\frac{2 M}{r}\right) \frac{h_{1}(r, t)}{r}
$$

resultam na equação

$$
-\frac{\partial^{2} \psi}{\partial t^{2}}+\frac{\partial^{2} \psi}{\partial x^{2}}-V(r) \psi=0
$$

onde $x(r)=r+2 M \log \left(\frac{r}{2 M}-1\right)$ é a coordenada tartaruga e o potencial efetivo é dado por

$$
V(r)=\left(1-\frac{2 M}{r}\right)\left[\frac{l(l+1)}{r^{2}}+\frac{2 \sigma M}{r^{3}}\right]
$$

com $\sigma=1-s^{2}$, sendo $s$ o spin da perturbação. Este potencial é chamado de potencial de Regge-Wheeler.

Para perturbações axiais, a equação de perturbação final é a mesma equação para uma perturbação polar (equação (2.17)), com potencial efetivo dado em [12] expresso por

$$
V(r)=\left(1-\frac{2 M}{r}\right) \frac{2 n^{2} r^{3}+2(n r+3 M)^{3}}{r^{3}(n r+3 M)^{2}},
$$

$\operatorname{com} 2 n=(l-1)(l+2)$. 
O resultado mais importante de toda a manipulação matemática feita nesta seção é que a estabilidade da métrica, que a rigor é feita com perturbações de uma matriz, pode ser reduzida a uma equação de perturbação de um campo escalar, cuja solução é mais simples. Supondo uma dependência temporal do tipo $\psi(x, t)=$ $e^{i \omega t} \psi(x)$ na equação (2.17), podemos reescrevê-la como

$$
\frac{\partial^{2} \psi}{\partial x^{2}}+\left(\omega^{2}-V(x)\right) \psi=0 .
$$

Para potenciais que tendem a zero quando $x$ tende a $\pm \infty$, como os potenciais de Regge-Wheeler e de Zerilli (equações (2.18) e (2.19)), as soluções assintóticas de $\psi(x)$ devem ser $\psi(x) \simeq e^{ \pm i \omega x}$. Desprezando soluções com fontes vindo do infinito espacial $(x \rightarrow \infty)$, pois queremos um sistema isolado de um buraco negro e sua perturbação, e vindo do horizonte de eventos $(x \rightarrow-\infty)$, pois supomos que nada possa sair do horizonte de eventos, temos que as soluções assintóticas de $\psi(x)$ devem ser $\psi(x)=e^{i \omega x}$ para $x \rightarrow \infty$ e $\psi(x)=e^{-i \omega x}$ para $x \rightarrow-\infty$.

\subsection{Estabilidade dos modos quasinormais}

Vishveshwara e Price já mostraram em [13; 14] que um buraco negro de Schwarzschild é estável, isto é, pequenas perturbações não crescem no tempo a ponto de se tornarem maior que os termos da métrica não perturbada. Wald em [15] prova a estabilidade de uma maneira mais rigorosa. Devemos notar que as equações de perturbação envolvem termos lineares da métrica, e a prova de que estas equações são estáveis não prova que o espaço-tempo é estável se as perturbações de ordens mais altas forem instáveis.

Wald reescreve a equação de perturbação (2.17) como

$$
\frac{\partial^{2} \psi}{\partial t^{2}}+A \psi=0
$$

sendo $A$ um operador positivo definido dado por

$$
A=-\frac{\partial^{2}}{\partial x^{2}}+V .
$$

Multiplicando a equação (2.21) por $\dot{\bar{\psi}}(x, t)$ e integrando de $-\infty$ a $+\infty$ em $x$, temos

$$
\int \dot{\bar{\psi}} \ddot{\psi} \mathrm{d} x+\int \dot{\bar{\psi}} A \psi \mathrm{d} x=0 \text {. }
$$

Somando a esta equação o seu complexo conjugado, que também deve ser nulo, e usando o fato de $A$ ser um operador auto-adjunto, temos

$$
\frac{\partial}{\partial t}\left(\int|\dot{\bar{\psi}}|^{2} \mathrm{~d} x+\int \bar{\psi} A \psi \mathrm{d} x\right)=0 \text {. }
$$


Como o operador $A$ é positivo definido, a segunda integral é real e positiva. A primeira integral é então limitada e os modos quasinormais não podem crescer exponencialmente, mas a equação acima não impede que os modos cresçam lentamente.

Em [15], Wald mostra que se $f(x)$ for uma função contínua e infinitamente diferenciável com suporte compacto, então para qualquer valor de $x$,

$$
|f(x)|^{2}<\frac{1}{2}\left[\int|f(x)|^{2} \mathrm{~d} x+\int\left|f^{\prime}(x)\right|^{2} \mathrm{~d} x\right] .
$$

Aplicando este lema na solução $\psi(x, t)$, temos

$$
|\psi(x, t)|^{2}<\frac{1}{2} \int|\psi(x, t)|^{2} \mathrm{~d} x+\frac{1}{2} \int\left|\psi^{\prime}(x, t)\right|^{2} \mathrm{~d} x .
$$

Como o potencial é positivo definido,

$$
\int\left|\psi^{\prime}(x, t)\right|^{2} \mathrm{~d} x<\int \bar{\psi} A \psi \mathrm{d} x .
$$

Substituindo as equações (2.26) e (2.27) na integral da equação (2.24),

$$
\int \bar{\psi}(x, t) A \psi(x, t) \mathrm{d} x \leq \int \bar{\psi}\left(x, t_{0}\right) A \psi\left(x, t_{0}\right) \mathrm{d} x+\int\left|\dot{\psi}\left(x, t_{0}\right)\right|^{2} \mathrm{~d} x,
$$

onde usamos o fato de que a integral da equação (2.24) é constante para substituir $t_{0}$ no lugar de $t$.

Combinando as desigualdades desta seção, podemos finalmente expressar um limite para a função $\psi(x, t)$ dado por

$$
|\psi(x, t)|^{2}<\int\left|\psi\left(x, t_{0}\right)\right|^{2} \mathrm{~d} x+\frac{1}{2} \int \bar{\psi}\left(x, t_{0}\right) A \psi\left(x, t_{0}\right) \mathrm{d} x+\frac{3}{2} \int\left|\dot{\psi}\left(x, t_{0}\right)\right|^{2} \mathrm{~d} x .
$$

O lado direito da equação (2.29) é positivo e finito, então se as condições iniciais $\psi\left(x, t_{0}\right)$ e $\psi\left(x, t_{0}\right)$ forem contínuas, infinitamente diferenciáveis com suporte compacto e $A$ for positivo definido, ou seja, se o potencial $V(x)$ for positivo definido, o valor de $|\psi(x, t)|^{2}$ é limitado para todo $t>t_{0}$.

Horowitz e Hubeny fazem em [16] uma conta semelhante para obter um critério de estabilidade de um modo quasinormal de um buraco negro em um espaçotempo assintoticamente AdS em $d$ dimensões esfericamente simétrico.

Pode-se reescrever a métrica do buraco negro em coordenadas de EddingtonFinkelstein, vista no capítulo 1,

$$
\mathrm{d} s^{2}=-f(r) \mathrm{d} v^{2}+2 \mathrm{~d} v \mathrm{~d} r+r^{2} \mathrm{~d} \Omega_{d-2}^{2},
$$

onde $v=t+x$ e $\mathrm{d} \Omega_{d-2}^{2}$ é a métrica de curvatura constante dos $d-2$ ângulos. 
A equação escalar que resulta da perturbação da métrica, após uma separação de variáveis do tipo

$$
\Phi\left(v, r, \theta_{1}, \cdots, \theta_{d-2}\right)=e^{-i \omega v} r^{\frac{2-d}{2}} \psi(r) Y\left(\theta_{1}, \cdots, \theta_{d-2}\right)
$$

é dada por

$$
\frac{\mathrm{d}}{\mathrm{d} r}\left[f(r) \frac{\mathrm{d} \psi}{\mathrm{d} r}\right]-2 i \omega \frac{\mathrm{d} \psi}{\mathrm{d} r}-V(r) \psi=0
$$

onde $V(r)$ é o potencial efetivo.

Multiplicando a equação (2.32) por $\bar{\psi}(r)$ e integrando do horizonte de eventos $r_{+}$até $\infty$, temos

$$
\int_{r_{+}}^{\infty}\left\{\bar{\psi} \frac{\mathrm{d}}{\mathrm{d} r}\left[f(r) \frac{\mathrm{d} \psi}{\mathrm{d} r}\right]-2 i \omega \bar{\psi} \frac{\mathrm{d} \psi}{\mathrm{d} r}-V(r) \bar{\psi} \psi\right\} \mathrm{d} r=0
$$

Integrando o primeiro termo por partes, temos um termo de superfície nulo, pois $f\left(r_{+}\right)=0$ por definição de $r_{+}$e $\lim _{r \rightarrow \infty} \psi(r)=0$ por condição de contorno, pois o potencial efetivo diverge para $r \rightarrow \infty$. A equação (2.33) é escrita como

$$
\int_{r_{+}}^{\infty}\left[f(r)\left|\psi^{\prime}\right|^{2}+2 i \omega \bar{\psi} \psi^{\prime}+V|\psi|^{2}\right] \mathrm{d} r=0
$$

Analisando apenas a parte imaginária da equação (2.34), temos

$$
\int_{r_{+}}^{\infty}\left[\omega \bar{\psi} \psi^{\prime}+\bar{\omega} \psi \bar{\psi}^{\prime}\right] \mathrm{d} r=0
$$

cujo segundo termo pode ser integrado por partes, resultando em

$$
(\omega-\bar{\omega}) \int_{r_{+}}^{\infty} \bar{\psi} \psi^{\prime} \mathrm{d} r=\bar{\omega}\left|\psi\left(r_{+}\right)\right|^{2}
$$

Usando a expressão (2.36) para substituir a integral de $\bar{\psi} \psi^{\prime}$ em (2.33), temos

$$
\int_{r_{+}}^{\infty}\left[f(r)\left|\psi^{\prime}\right|^{2}+V(r)|\psi|^{2}\right] \mathrm{d} r=-\frac{|\omega|^{2}\left|\psi\left(r_{+}\right)\right|^{2}}{\operatorname{Im}(\omega)} .
$$

A função $f(r)$ é, por definição, positiva definida em $\left(r_{+}, \infty\right)$. Se $V(r)$ também for positivo definido em $\left(r_{+}, \infty\right)$, os valores de $\omega$ permitidos são tais que $\operatorname{Im}(\omega)<$ 0 , que pela separação de variáveis (2.31), correspondem a modos estáveis. 


\subsection{Método WKB}

A motivação de Schutz e Will em [17] para aplicar o método WKB (Wentzel, Kramers e Brillouin) é a semelhança entre a equação

$$
\frac{\mathrm{d}^{2} \psi}{\mathrm{d} x^{2}}+Q(x) \psi=0
$$

com a equação de Schrödinger, com $-Q(x)=\left(\frac{2 m}{\hbar^{2}}\right)[V(x)-E]$. Com uma dependência $\psi(r, t)=e^{i \omega t} \psi(r)$, a equação (2.17) pode ser escrita na forma da equação acima com $Q(x)=\omega^{2}-V(x)$.

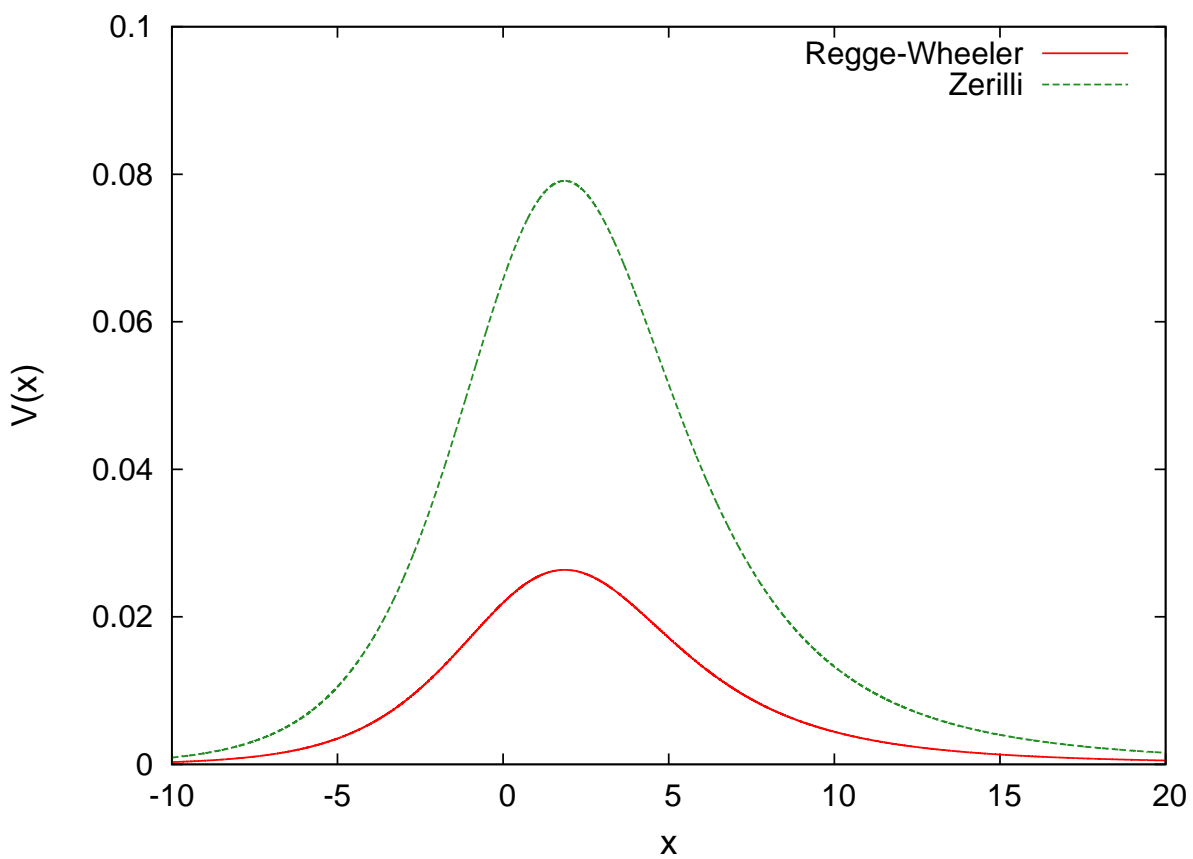

Figura 2.1: Exemplo de potenciais de Regge-Wheeler e Zerilli $\operatorname{com} M=1$.

Sejam $x_{1}$ e $x_{2}$ os pontos em que $Q(x)=0$ e $x_{0}$ o ponto de máximo de $V(x)$. Para $x>x_{2}, \psi(x)$ é dado por

$$
\psi(x) \simeq Q^{-\frac{1}{4}} \exp \left\{ \pm i \int_{x_{2}}^{x} \sqrt{Q(t)} \mathrm{d} t\right\}
$$

e para $x<x_{1}$, por

$$
\psi(x) \simeq Q^{-\frac{1}{4}} \exp \left\{ \pm i \int_{x}^{x_{1}} \sqrt{Q(t)} \mathrm{d} t\right\}
$$


Entre $x_{1}$ e $x_{2}, Q(x)$ pode ser aproximado por uma parábola, e a solução para $\psi(x)$ é dada por funções parabólicas cilíndricas cuja expressão assintótica só "encaixa" com as expressões (2.39) e (2.40) se

$$
\frac{Q_{0}}{\sqrt{2 Q_{0}^{\prime \prime}}}=i\left(n+\frac{1}{2}\right),
$$

onde $\left.Q_{0}=Q_{(} x_{0}\right), Q_{0}^{\prime \prime}=Q^{\prime \prime}\left(x_{0}\right)$ e $n$ é um número inteiro.

Em [18], Iyer e Will usam aproximações de ordens mais altas para o potencial em torno do máximo para corrigir a equação (2.41). Esta correção é utilizada em [19] para se obterem valores de frequências quasinormais de buracos negros de Schwarzschild, em [20] para buracos negros de Reissner-Nordström e em [21] para buracos negros de Kerr.

Este método é chamado também de método semi-analítico porque, apesar de apresentar expressões analíticas para as aproximações dos cálculos de frequências, os cálculos devem ser feitos em um ponto de máximo do potencial que deve ser obtido numericamente. O cálculo numérico pode ser feito utilizando algum método de busca de soluções para $V^{\prime}(x)=0$.

\subsection{Método de Horowitz-Hubeny}

O método WKB só pode ser utilizado para equações cujo potencial efetivo tende a zero quando $x$ tende a $-\infty \mathrm{e}+\infty$. Essa condição não é satisfeita em espaços-tempos assintoticamente anti-de Sitter. Neste caso, usa-se um método definido em [16] e conhecido como Método de Horowitz-Hubeny. Escrevendo a métrica de Schwarzschild-AdS em termos de coordenadas de Eddington-Finkelstein $v=t+x$ temos

$$
\mathrm{d} s^{2}=-f(r) \mathrm{d} v^{2}+2 \mathrm{~d} v \mathrm{~d} r+r^{2} \mathrm{~d} \Omega_{2}^{2} .
$$

Com a separação de variáveis $\Psi(v, r, \theta, \varphi)=\frac{Z(r)}{r} e^{-i \omega v} Y_{l m}(\theta, \varphi)$ a equação de movimento para $Z(r)$ assume a forma

$$
f(r) \frac{\mathrm{d}^{2} Z(r)}{\mathrm{d} r^{2}}+\left[f^{\prime}(r)-2 i \omega\right] \frac{\mathrm{d} Z}{\mathrm{~d} r}-V(r) Z=0
$$

onde $V(r)$ é o potencial efetivo.

Seguindo a receita dada em [16], com a transformação de coordenadas $x=\frac{1}{r}$, escrevemos (2.43) como

$$
s(x) \frac{\mathrm{d}^{2} Z}{\mathrm{~d} x^{2}}+\frac{t(x)}{\left(x-x_{+}\right)} \frac{\mathrm{d} Z}{\mathrm{~d} x}+\frac{u(x)}{\left(x-x_{+}\right)^{2}} Z=0,
$$


com

$$
\begin{aligned}
s(x) & =\frac{\left(x_{+}^{2}+1\right) \frac{x^{d+1}}{x_{+}^{d-1}}-x^{4}-x^{2}}{x-x_{+}} \\
t(x) & =(d-1)\left(x_{+}^{2}+1\right) \frac{x^{d}}{x_{+}^{d-1}}-2 x^{3}-2 x^{2} i \omega, \\
u(x) & =\left(x-x_{+}\right) V(x) .
\end{aligned}
$$

Os polinômios $s(x), t(x)$ e $u(x)$, expandidos em torno de $x=x_{+}$, geram uma série finita expressa por

$$
s(x)=\sum_{n=0}^{d} s_{n}\left(x-x_{+}\right)^{n}
$$

e expressões equivalentes para $t_{n}$ e $u_{n}$.

Expandindo $Z(x)$ em torno de $x_{+}$, temos

$$
Z(x)=\sum_{n=0}^{\infty} a_{n}(\omega)\left(x-x_{+}\right)^{n} .
$$

com

$$
\begin{aligned}
& a_{n}=-\frac{1}{P_{n}} \sum_{k=0}^{n-1}\left[k(k-1) s_{n-k}+k t_{n-k}+u_{n-k}\right] a_{k}, \\
& P_{n}=n(n-1) s_{0}+n t_{0} .
\end{aligned}
$$

As frequências dos modos quasinormais são calculadas obtendo as raízes da equação $Z(x=0)=0$, que é um polinômio de grau infinito em $\omega$. Na prática, truncamos o polinômio em um grau $N$ suficientemente grande e vemos se ao aumentarmos o valor de $N$ a frequência fundamental converge para algum valor. $\mathrm{O}$ cálculo das raízes pode ser feito com algum método de busca de soluções, porém a busca deve levar em conta que as raízes são complexas. Por se tratar de um polinômio, pode-se usar o método de Jenkins-Traub (seção A.1.6) ou o método de Laguerre (seção A.1.5). No entanto não é provado que este último método funcione para busca de raízes complexas.

\subsection{Integração numérica}

As equações de perturbações para calcular os modos quasinormais são equações diferenciais parciais de segunda ordem, cuja solução pode ser obtida pelo método de diferenças finitas, que pode ser visto na seção A.4.1. Este método é 
mais rudimentar que os outros métodos disponíveis, no entanto ele depende de menos condições. Na prática, as funções que aparecem na equação diferencial devem ser contínuas e duas vezes diferenciáveis. Para uma perturbação em um espaço-tempo esfericamente simétrico (e portanto, sem rotação), esta condição quer dizer que o domínio de integração (i.e., região do espaço-tempo a ser dividida em intervalos constantes) deve estar fora do horizonte de eventos.

Como o sinal de $g_{00}$ é diferente do sinal de $g_{11}$, a equação de perturbação após a separação das variáveis angulares por harmônicos esféricos, será uma equação hiperbólica. Devem-se então definir duas condições iniciais, por exemplo, $\psi\left(x, t_{0}\right)=f(x)$ e $\dot{\psi}\left(x, t_{0}\right)=g(x)$ e duas condições de contorno. Em espaçostempos assintoticamente AdS, temos a condição de contorno de $\psi(x, t)=0$ para $x=0$, pois o valor de $x=0$ corresponde a $r$ tendendo ao infinito. Como o potencial efetivo diverge para $+\infty$, podemos escolher a condição de contorno de que os modos devem tender a zero. No entanto, não temos uma condição física para atribuir à outra fronteira da grade numérica, que deve ser um valor finito. Em espaçostempos assintoticamente plano, as duas condições de contorno ficam difíceis de serem atribuídas, pois qualquer valor finito da coordenada tartaruga corresponde a um valor finito de $r$ maior que o horizonte de eventos.

Não ter uma condição física para atribuir a um valor finito das coordenadas não é um problema se tomarmos um domínio de integração suficientemente grande na coordenada $x$ de modo que o comportamento dos modos quasinormais seja observado dentro do domínio de Cauchy (ver referências [22; 23]), dentro do qual a solução numérica da equação de perturbação depende apenas das condições iniciais do problema.

Definida a grade numérica como $x=x_{0}+j \cdot \Delta x$ e $t=t_{0}+l \cdot \Delta t$, reescrevemos a equação

$$
-\frac{\partial^{2} \psi}{\partial t^{2}}+\frac{\partial^{2} \psi}{\partial x^{2}}-V \psi=0
$$

como

$$
-\frac{\psi_{j, l+1}-2 \psi_{j, l}+\psi_{j, l-1}}{\Delta t^{2}}+\frac{\psi_{j+1, l}-2 \psi_{j, l}+\psi_{j-1, l}}{\Delta x^{2}}-V_{j} \psi_{j, l}=0 .
$$

As condições iniciais $\psi\left(x, t_{0}\right)=f(x)=f_{j}$ e $\dot{\psi}\left(x, t_{0}\right)=g(x)=g_{j}$ determinam o valor de $p s i_{j, 0}=f_{j}$ e $\psi_{j, l}=f_{j}+g_{j} \Delta t$, e partindo destes valores, calculamos o valor de $\psi_{j, l}$ para $l>1$ por

$$
\psi_{j, l+1}=-\psi_{j, l-1}+\frac{\Delta t^{2}}{\Delta x^{2}}\left(\psi_{j+1, l}+\psi_{j-1, l}\right)+\left(2-2 \frac{\Delta t^{2}}{\Delta x^{2}}-V_{j}\right) \psi_{j, l} .
$$

O critério de estabilidade de von Neumann (seção A.4.3) ${ }^{1}$ aplicado à equação

\footnotetext{
${ }^{1}$ Não confundir com os critério de estabilidade apresentados neste capítulo. O critério de von
} 
(2.54) resulta em

$$
\operatorname{sen}^{2}\left(\frac{v}{2}\right)=\frac{\Delta t^{2}}{\Delta x^{2}} \operatorname{sen}^{2}\left(\frac{\theta}{2}\right)+\frac{\Delta t^{2}}{4} V(\theta) .
$$

O método numérico é estável se $v$ só tiver soluções com parte imaginária maior ou igual a zero para qualquer valor real de $\theta$. Se o lado direito da equação (2.55) for menor que 1, os valores possíveis de $v$ são todos reais, se for maior que 1 , as soluções para $v$ são pares de complexos conjugados, e o método numérico é portanto instável.

Para garantir a estabilidade do método para qualquer valor de $\theta$ real, seja $V_{\mathrm{MAX}}$ 2 o maior valor que $V(r)$ pode assumir na grade numérica. Logo

$$
\frac{\Delta t^{2}}{\Delta x^{2}} \operatorname{sen}^{2}\left(\frac{\theta}{2}\right)+\frac{\Delta t^{2}}{4} V(\theta)<\frac{\Delta t^{2}}{\Delta x^{2}}+\frac{\Delta t^{2}}{4} V_{\text {MAX }}<1 .
$$

Para potenciais como os de Regge-Wheeler e Zerilli (equações (2.18) e (2.19)) que possuem um máximo em um valor finito, basta que $\Delta t$ seja pequeno se comparado a $V_{\text {MAX }}$ e menor que $\Delta x$. Em casos em que o potencial efetivo diverge quando

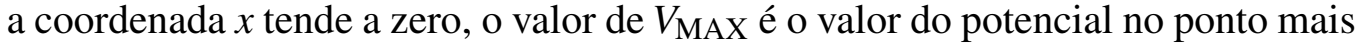
próximo a $x=0$ na grade numérica. Se o método numérico for instável devido ao potencial, é possível diminuir o passo $\Delta t$, ou aumentar o passo $\Delta x$, o que afasta o ponto adjascente a $x=0$ da grade numérica e diminui o valor de $V_{\mathrm{MAX}}$. Esta última solução é melhor se o potencial divergir com uma potência maior que $r^{2}$.

\footnotetext{
Neumann mostra que um método numérico é uma aproximação para a solução da equação diferencial, enquanto os métodos apresentados na seção A.4.3 mostram que a solução da equação diferencial não crescem exponencialmente com o tempo.

${ }^{2} V_{\mathrm{MAX}}$ não é necessariamente o máximo do potencial como necessário no método WKB, apenas o valor do potencial no ponto da grade numérica mais próximo do ponto de máximo de $V(r)$.
} 


\section{Capítulo 3}

\section{Correspondência AdS/CFT}

Este capítulo trata de um tema complexo e não temos a pretensão de apresentar uma boa explicação como este tema merece. Nossa intenção é apenas selecionar tópicos relevantes para o capítulo 4 , que são essencialmente apresentar o conceito de um supercondutor holográfico e a relevância dos modos quasinormais para este tema. Recomendamos $[24 ; 25 ; 26 ; 27]$ como referências para este tema.

\subsection{Motivações e origens da correspondência}

Uma teoria de campos é dita Teoria de Campo Conforme (ou Conformal Field Theory) se for invariante por transformações conformes, que são generalizações da transformação de escala. Em $d$ dimensões, estas transformações são definidas tais que

$$
\mathrm{d} x_{\mu}^{\prime} \mathrm{d} x_{v}^{\prime}=[\Omega(x)]^{-2} \mathrm{~d} x_{\mu} \mathrm{d} x_{v} .
$$

Junto com o grupo de Poincaré, as tranformações definidas por (3.1) formam o grupo de simetria conforme $\mathrm{SO}(2, d)$, que é o mesmo grupo de simetria do espaço AdS em $d+1$ dimensões, como visto no capítulo 1 .

Em [28], t' Hooft mostra que uma teoria de calibre $S U(N)$, onde $N$ é o número de cores, para $N$ grande é relacionada com a teoria de cordas. Essa relação é mais evidente com as D-branas [29], que são objetos que generalizam o conceito de cordas. Em [24], é dito que uma D-brana corresponde a uma solução de supergravidade da qual pode-se obter uma radiação Hawking a partir de um processo em que duas cordas abertas colidem, formam uma corda fechada e se desprendem da D-brana. Em uma teoria com D3-branas, cordas abertas com pontas na brana dão origem a uma teoria que se reduz a Super-Yang-Mills $\mathcal{N}=4$ em um limite de baixas energias, cordas fechadas (que são separadas da brana) dão origem a uma supergravidade acoplada com modos massivos da corda, e interações podem levar 
um tipo de corda na outra. Em um limite apropriado de baixas energias, não há interação entre os tipos de corda.

Maldacena em [30] mostra que a teoria Super-Yang-Mills $\mathcal{N}=4$ na descrição em termos de D-branas é dual a uma teoria de supercordas tipo IIB em $\mathrm{AdS}_{5} \times \mathrm{S}_{5}$, de onde pode-se obter uma solução expressa por

$$
\mathrm{d} s^{2}=\frac{r^{2}}{L^{2}}\left(-\mathrm{d} t^{2}+\sum_{i=1}^{3} \mathrm{~d} x_{i}^{2}\right)+\frac{L^{2}}{r^{2}} \mathrm{~d} r^{2}+L^{2} \mathrm{~d} \Omega_{5}^{2} .
$$

que por uma transformação $\frac{r}{L}=\frac{L}{z}$, é escrita como

$$
\mathrm{d} s^{2}=\frac{L^{2}}{z^{2}}\left(-\mathrm{d} t^{2}+\sum_{i=1}^{3} \mathrm{~d} x_{i}^{2}+\mathrm{d} z^{2}\right)+L^{2} \mathrm{~d} \Omega_{5}^{2}
$$

que é a métrica do espaço $A d S_{5} \times S_{5}$ vista no capítulo 1 , com a métrica de uma 5 -esfera de raio $L$. A teoria conforme relacionada à supergravidade em AdS é definida em $z=0$, que corresponde ao infinito espacial.

Em [31], Witten mostra uma relação entre funções de correlação em uma teoria de campo conforme em $d$ dimensões e o comportamento da ação da supergravidade em $(d+1)$ dimensões no infinito espacial. Esta relação compara a dimensão do operador na teoria de campo conforme com massas de partículas na supergravidade.

\subsection{Princípio holográfico}

Em [25], Aharony et al. descrevem como a correspondência AdS/CFT se relaciona com uma descrição holográfica em espaços AdS. Para evitar violações da segunda lei da termodinâmica generalizada de Bekenstein, é dito que em uma teoria de gravitação quântica, toda a física contida em um certo volume pode ser descrita em termos de uma teoria na fronteira deste volume, que tem um grau de liberdade por área de Planck [32; 33].

Seja a métrica do espaço $\mathrm{AdS}_{5}$ dada por

$$
\mathrm{d} s^{2}=L^{2}\left[-\left(\frac{1+r^{2}}{1-r^{2}}\right)^{2} \mathrm{~d} t^{2}+\frac{4}{\left(1-r^{2}\right)^{2}}\left(\mathrm{~d} r^{2}+r^{2} \mathrm{~d} \Omega_{3}^{2}\right)\right],
$$

onde a fronteira AdS está localizada em $r=1$. A métrica (3.4), para valores de $r$ próximos a 1, pode ser escrita como

$$
\mathrm{d} s^{2}=\frac{L^{2}}{y^{2}}\left(-\mathrm{d} t^{2}+\mathrm{d} x^{i} \mathrm{~d} x^{i}+\mathrm{d} y^{2}\right)
$$


onde temos uma soma com $i$ variando de 1 a 3 . Com uma transformação de coordenadas $z=\frac{1}{y}$, escrevemos a equação (3.5) como

$$
\mathrm{d} s^{2}=L^{2}\left(-z^{2} \mathrm{~d} t^{2}+z^{2} \mathrm{~d} x^{i} \mathrm{~d} x^{i}+\frac{1}{z^{2}} \mathrm{~d} z^{2}\right)
$$

sendo que agora a fronteira AdS está localizada em $z=\infty$.

$\mathrm{O}$ espaço $\mathrm{AdS}_{5} \times \mathrm{S}_{5}$ é construído definindo 5 coordenadas que definem o espaço $\mathrm{S}_{5}$ com a métrica

$$
\mathrm{d} s^{2}=L^{2} \mathrm{~d} \Omega_{5}^{2}
$$

Seja uma superfície $\mathcal{L}$ definida em $r=1-\delta$, cujo volume, se calculado pela métrica (3.4) é proporcional a $L^{9} / \delta^{3}$ e o número de graus de liberdade é proporcional a $L^{9} / l_{p}^{9} \delta^{3}$, onde $l_{p}$ é o comprimento de Planck. Na fronteira $\mathcal{L}$, como visto em [27], a entropia é limitada por $A / l_{p}^{8}$, onde $A$ é a área de $\mathcal{L}$, ou seja,

$$
S_{\mathrm{MAX}} \sim \frac{1}{\delta^{3}} \frac{L^{8}}{l_{p}^{8}} .
$$

Para uma razão $L / l_{p}$ grande, a descrição holográfica requer uma redução no número de graus de liberdade por um fator $l_{p} / L$. Em outras palavras, o princípio holográfico dá uma descrição da física em uma região de um espaço $\mathrm{AdS}$ em termos de $l_{p} / L$ graus de liberdade por volume de Planck.

\subsection{Alguns "verbetes" do dicionário AdS/CFT}

A correspondência AdS/CFT fala especificamente de uma relação entre uma teoria de cordas em $\mathrm{AdS}_{5} \times \mathrm{S}^{5}$ e uma teoria Super Yang Mills definida em uma fronteira. Para relacionar quantidades físicas entre as duas teorias, é feita uma série de comparações que pode ser entendida como um dicionário. É conveniente, segundo [25; 27], que as coordenadas definidas na teoria localizada na borda sejam adimensionais, assim como os momentos. A energia definida na teoria de Yang Mills deve então estar relacionada com a energia definida no espaço AdS (ver capítulo 1) como $E_{\mathrm{SYM}}=M L$.

Entre outros exemplos, estão relacionadas a constante de acoplamento das cordas e a constante de Newton por

$$
G=g^{2} l_{s}^{8}
$$

onde $l_{s}$ é a escala de comprimento da teoria de cordas. Em [30], Maldacena mostra relações entre a constante de acoplamento na teoria de Yang-Mills $g_{Y M}$ e o posto 
do grupo de gauge $N$, dadas por

$$
\begin{aligned}
\frac{L}{l_{S}} & =\left(N g_{Y M}^{2}\right)^{\frac{1}{4}}, \\
g & =g_{Y M}^{2} .
\end{aligned}
$$

A relação mais útil entre as duas teorias é, provavelmente, uma relação entre a função de partição da teoria de cordas em $\mathrm{AdS}_{5} \times \mathrm{S}^{5}$ e a função de partição da teoria Super-Yang-Mills definida na teoria de campo dual. Em um limite com $N$ grande e $g_{Y M}^{2} N$ fixo, a teoria de cordas é bem aproximada por uma teoria de gravitação clássica, e a relação entre as funções de partição pode ser escrita como

$$
e^{-I_{\mathrm{G}}} \simeq Z_{\mathrm{corda}}=Z_{\mathrm{gauge}}=e^{-W}
$$

onde $W$ é o gerador da função de Green na teoria de calibre. Para temperaturas finitas, $Z=\beta F$, sendo $\beta$ o inverso da temperatura e $F$ a energia livre.

De acordo com a correspondência AdS/CFT, valores de contornos de campos na teoria de cordas agem como fontes de operadores. Seja um campo clássico definido na teoria de cordas denotado por $\phi\left(x^{i}, y\right)$, de acordo com o sistema de coordenadas (3.5), com o valor $\phi_{0}\left(x^{i}\right)$ quando $y=\varepsilon$, com $\varepsilon$ próximo de 0 , que é a fronteira AdS no sistema de coordenadas citado.

Escolhendo valores de $\phi_{0}\left(x^{i}\right)$ e calculando os extremos de $I_{\mathrm{G}}[\phi]$ em $y>\varepsilon$ sujeitos à escolha $\phi_{0}\left(x^{i}\right)$, pode-se escrever

$$
W\left[\phi_{0}\right]=-\log \left\langle e^{\int d^{4} x \phi_{0}(x) O(x)}\right\rangle_{\mathrm{CFT}} \simeq \underset{\phi(x)=\phi_{0}(x)}{\operatorname{extremo}} I_{\mathrm{G}}[\phi]
$$

lembrando que $W\left[\phi_{0}\right]$ é o gerador da função de Green na teoria de campo. $\phi_{0}$ é uma função arbitrária e podemos calcular funções de correlação tomando derivadas funcionais em relação a $\phi_{0} \operatorname{com} \phi_{0}=0$. O lado direito da equação (3.13) depende da função de partição da teoria de cordas com a condição de contorno $\phi=\phi_{0}$ na fronteira AdS.

Utilizando a equação (3.13), um buraco negro imerso em um espaço AdS é mapeado em um estado térmico na teoria de campo dual, com temperatura igual à temperatura Hawking do buraco negro assintoticamente AdS. Uma perturbação do buraco negro é mapeada em um campo escalar que, sob temperaturas menores que um valor crítico, se comporta como um parâmetro de ordem que descreve uma transição de fase. Por este mapeamento ser feito teorias definidas em um número diferentes de dimensões, a transição de fase descreve um supercondutor holográfico. 


\subsection{Supercondutores holográficos}

Supercondutores holográficos aparecem em teorias de campos que exibem uma transição de fase abaixo de uma certa temperatura e que apresentam um dual gravitacional de acordo com a correspondência AdS/CFT. Este tema é explicado com mais detalhes em [26; 34]. Na teoria de campo, a supercondutividade é caracterizada por uma condensação de um operador $O$ para temperaturas menores que uma temperatura crítica. Na teoria dual gravitacional, a transição para a supercondutividade é observada como uma instabilidade clássica de um buraco negro assintoticamente AdS por perturbações de um campo escalar carregado $\psi$. A instabilidade deve aparecer quando a temperatura de Hawking do buraco negro é menor que a temperatura crítica.

A lagrangiana mais simples que descreve os fenômenos de interesse é

$$
\mathfrak{L}=R+\frac{6}{L^{2}}-\frac{1}{4} F^{\mu v} F_{\mu v}-m^{2}|\psi|^{2}-\left|\left(\nabla_{\mu}-i q A_{\mu}\right) \psi\right|^{2}
$$

onde $q$ é a carga do campo escalar $\psi$ e $m^{2}$ é a massa ao quadrado. ${ }^{1}$

As equações de movimento para $\psi$ são dadas por

$$
-\square \psi-2 i q A_{\mu} \nabla^{\mu} \psi+q^{2} A_{\mu} A^{\mu} \psi+m^{2} \psi=0,
$$

as equações de Maxwell por

$$
\nabla^{\mu} F_{\mu \nu}=i q\left[\bar{\psi}\left(\nabla_{v}-i q A_{v}\right) \psi-\psi\left(\nabla_{\nu}+i q A_{v}\right) \bar{\psi}\right]
$$

e as equações de Einstein por

$$
\begin{gathered}
R_{\mu \nu}-\frac{1}{2} R g_{\mu \nu}-\frac{3}{L^{2}} g_{\mu \nu}=\frac{1}{2} F_{\mu \alpha} F_{v}^{\alpha}-\frac{1}{8} g_{\mu \nu} F^{\alpha \beta} F_{\alpha \beta} \\
-\frac{1}{2} g_{\mu v} m^{2}|\psi|^{2}-\frac{1}{2} g_{\mu \nu}\left|\nabla_{\alpha} \psi-i q A_{\alpha} \psi\right|^{2} \\
+\frac{1}{2}\left[\left(\nabla_{\mu}-i q A_{\mu} \psi\right)\left(\nabla_{v} \bar{\psi}+i q A_{v} \bar{\psi}\right)\left(\nabla_{v}-i q A_{\nu} \psi\right)\left(\nabla_{\mu} \bar{\psi}+i q A_{\mu} \bar{\psi}\right)\right] .
\end{gathered}
$$

As equações (3.15), (3.16) e (3.17) descrevem as fases da teoria. Uma transição de fase deve ser observada ao diminuir a temperatura, supondo que exista uma temperatura Hawking, ou seja, deve existir um horizonte de eventos.

Seja um buraco negro definido pela métrica

$$
\mathrm{d} s^{2}=-f(r) \mathrm{d} t^{2}+\frac{1}{f(r)} \mathrm{d} r^{2}+r^{2} \mathrm{~d} \Omega^{2}
$$

\footnotetext{
${ }^{1} \mathrm{O}$ escalar de Ricci e a constante cosmológica não aparecem com um termo de $16 \pi G$ por uma redefinição conveniente de $A_{\mu}$ e $\Psi$.
} 
com as imposições $A_{\mu} \mathrm{d} x^{\mu}=\Phi(r) \mathrm{d} t$ e $\psi=\psi(r)$. A componente 1 das equações de Maxwell implica em uma fase constante para $\psi(r)$, que pode ser tomado como real. A equação de movimento para $\psi$ pode ser escrita como

$$
\psi^{\prime \prime}+\left(\frac{f^{\prime}(r)}{f(r)}+\frac{2}{r}\right) \psi^{\prime}+\frac{q^{2} \Phi^{2}(r)}{f^{2}(r)} \psi-\frac{m^{2}}{f(r)} \psi=0
$$

e a equação de Maxwell para $\Phi(r)$ como

$$
\Phi^{\prime \prime}+\frac{2}{r} \Phi^{\prime}-\frac{2 q^{2} \Psi^{2}(r)}{f(r)} \Phi=0 .
$$

As equações (3.19) e (3.20) formam um sistema de duas equações diferenciais ordinárias acopladas, cuja solução pode ser obtida numericamente pelo método de Runge-Kutta (seção A.3.2) a partir de quatro condições para $\psi$ e $\Phi$ no horizonte de eventos. Para a forma $\Phi(r) \mathrm{d} t$ ter norma finita no horizonte, é necessário que $\Phi\left(r_{+}\right)$seja igual a zero. Para a equação (3.19) ser regular no horizonte, $\psi^{\prime}\left(r_{+}\right)=$ $\frac{m^{2}}{f^{\prime}\left(r_{+}\right)} \psi\left(r_{+}\right)$. Há uma liberdade de escolha do $\operatorname{par}\left(\psi\left(r_{+}\right), \phi^{\prime}\left(r_{+}\right)\right)$. As equações de movimento, para um valor de $r$ suficientemente grande, se comportam como

$$
\begin{aligned}
& \Phi(r)=\mu-\frac{\rho}{r}+\cdots, \\
& \psi(r)=\frac{\psi^{(+)}}{r^{\Delta_{+}}}+\frac{\psi^{(-)}}{r^{\Delta_{-}}}+\cdots,
\end{aligned}
$$

onde $\Delta_{ \pm}$é solução de $\Delta(\Delta-3)=m^{2} L^{2}$.

Trivialmente, $\Phi(r)=\mu-\frac{\rho}{r}$ e $\psi(r)$ são soluções das equações de movimento. Em [35] é provado que acima de uma certa temperatura, apenas a solução trivial é permitida. O argumento parte de desvios da solução trivial de $\Phi(r)$ e $\psi(r)$, cuja existência depende de pontos de retorno em $\psi(r)$, que só ocorrem se os parâmetros físicos do buraco negro obedecerem a uma condição que só é válida se a temperatura Hawking for menor que um valor crítico.

Com uma mudança de variáveis $z=\frac{r_{+}}{r}$, os comportamentos assintóticos (3.21) e (3.22) são dados por

$$
\begin{aligned}
\Phi(z) & =\mu-\frac{\rho}{r_{+}} z, \\
\psi(z) & =D_{+} z^{\Delta_{+}}+D_{-} z^{\Delta_{-}} .
\end{aligned}
$$

Em torno do horizonte de eventos $(z=1)$, as funções $\Phi(z)$ e $\psi(z)$ podem ser expandidas em uma série de potências. Ao impor que o comportamento da série expansão em série em torno de $z=1$ deve "colar" com o comportamento assintótico com $z=0$ em um ponto intermediário $0<z<1$, obtém-se um sistema de 
equações relacionando $D_{+}$e $D_{-}$com os parâmetros físicos do buraco negro. Impondo $D_{-}=0$ é possível escrever $D_{+}$em termos da temperatura. Voltando à expressão (3.22) com $\psi^{(-)}=0$, o termo $\psi^{(+)}$, segundo a correspondência AdS/CFT, está relacionado ao valor esperado de um operador $O$ com dimensão $\Delta_{+}$. Este valor esperado vale zero quando a temperatura do buraco negro é igual a uma certa temperatura crítica, com um comportamento expresso (ver referência [36]) por

$$
\frac{<O_{+}>}{T_{c}} \simeq \frac{T}{T_{c}}\left\{\left(\frac{T_{c}}{T}\right)^{d-2}\left[1-\left(\frac{T}{T_{c}}\right)^{d-2}\right]\right\}^{\frac{1}{2 \Delta_{+}}}
$$

se $T<T_{c}$ e $d$ é o número de dimensões do espaço-tempo. Se $T>T_{c},<O_{+}>=0$ pois acima desta temperatura apenas a solução trivial $\psi(r)=0$ é permitida.

$\mathrm{O}$ valor esperado $\left\langle\mathrm{O}_{+}\right\rangle$se comporta como um parâmetro de ordem, assumindo valores diferentes de zero para temperaturas menores que a temperatura crítica e zero para temperaturas maiores que a temperatura crítica, como na teoria de Ginzburg-Landau de um supercondutor. Por este comportamento semelhante a um supercondutor, é dito que o dual do campo escalar $\psi$ mapeado holograficamente na teoria de campo conforme segundo a correspondência AdS/CFT é um supercondutor holográfico.

Para interpretar os termos da expansão assintótica de $\Phi(r)$, é feita em [37] uma perturbação de um buraco negro carregado. Ao escrever o potencial eletromagnético, a componente temporal deste potencial se comporta como um termo que decai como lei de potência mais uma constante próximo à fronteira $\mathrm{AdS}$ e não corresponde a um campo na teoria dual, mas fixa a densidade de carga de um estado. Esta componente deve ser nula no horizonte de eventos, então é necessário que a constante à qual o potencial tende no infinito anule o termo que decai como lei de potência, o que pode ser entendido na teoria dual como uma soma de um potencial químico $\mu$.

Considerando uma componente espacial do potencial vetor diferente de zero, como $A_{x}$ por exemplo, há um comportamento assintótico semelhante ao de $\Phi(r)$, dado por $A_{x}=A_{x}^{(0)}+\frac{A_{x}^{(1)}}{r}$. Pela correspondência AdS/CFT, $A_{x}^{(1)}$ é identificado como uma densidade de corrente elétrica na teoria dual, e $A_{x}^{(0)}$ como o potencial vetor associado à densidade de corrente elétrica. Utilizando a lei de $\mathrm{Ohm}$, calcula-se a condutividade, que se comporta como um valor constante para uma temperatura maior que a temperatura crítica e mostra um gap de energia em temperaturas menores [26]. 


\subsection{Papel dos modos quasinormais na correspondên- cia AdS/CFT}

Um dos trabalhos pioneiros de modos quasinormais no contexto da correspondência AdS/CFT é o trabalho de Horowitz e Hubeny[16], onde os autores desenvolvem um método para calcular modos quasinormais de buracos negros assintoticamente AdS. Neste trabalho também é dito que segundo a correspondência AdS/CFT , um buraco negro no espaço AdS está associado a um estado térmico na teoria conforme dual, e os modos quasinormais, que podem ser entendidos como uma perturbação do buraco negro descrevendo o retorno à configuração inicial, estão associados na teoria dual a uma perturbação do estado térmico, com uma escala de tempo de retorno ao equilíbrio térmico associada ao decaimento dos modos quasinormais.

Com o objetivo de testar mais a fundo a relação de modos quasinormais com uma teoria de campo dual, Birmingham, Sachs e Solodukhin[38] calculam modos quasinormais de um buraco negro BTZ[39], definido em 3 dimensões e comparam o valor das frequências com polos da função de Green retardada correspondente na teoria de campo conforme definida em 2 dimensões.

A métrica do BTZ, em unidades em que o raio anti-de Sitter vale 1, é dada por

$$
\mathrm{d} s^{2}=-\operatorname{senh}^{2} \mu\left(r_{+} \mathrm{d} t-r_{-} \mathrm{d} \varphi\right)^{2}+\cosh ^{2} \mu\left(-r_{-} \mathrm{d} t+r_{+} \mathrm{d} \varphi\right)^{2}+\mathrm{d} \mu^{2}
$$

onde $r_{+}$e $r_{-}$são os horizontes internos e externos respectivamente e $\mu$ é uma função biunívoca de $r$.

A teoria conforme dual é dividida em dois detores independentes com temperaturas

$$
T_{ \pm}=\frac{r_{+} \pm r_{-}}{2 \pi}
$$

Pela correspondência $\mathrm{AdS}_{3} / \mathrm{CFT}_{2}$ há um operador $O$ na teoria dual para cada campo de spin $s$ propagando no espaço AdS, com pesos conformes $h_{+}$e $h_{-}$que obedecem às relações

$$
\begin{aligned}
& h_{+}+h_{-}=\Delta, \\
& h_{+}-h_{-}=s,
\end{aligned}
$$

onde $\Delta$ depende da massa do campo como $\Delta=1+\sqrt{1+m^{2}}$ para campos escalares e $\Delta=1+|m|$ para campos vetoriais e espinoriais. A função de Green retardada associada aos operadores $O$, após uma transformada de Fourier, é proporcional a

$$
\tilde{G} \sim \Gamma\left(h_{-}+i \frac{p_{1}}{2 \pi T_{-}}\right) \Gamma\left(h_{+}+i \frac{p_{2}}{2 \pi T_{+}}\right) \Gamma\left(h_{-}-i \frac{p_{1}}{2 \pi T_{-}}\right) \Gamma\left(h_{+}-i \frac{p_{2}}{2 \pi T_{+}}\right),
$$


onde $p_{1}=\frac{1}{2}(\omega-k)$ e $p_{1}=\frac{1}{2}(\omega+k)$

Restringindo os polos da expressão (3.30) ao semiplano com parte imaginária negativa, temos

$$
\omega_{ \pm}=\mp k-4 \pi i T_{ \pm}\left(n+h_{ \pm}\right),
$$

onde $n$ é um número inteiro.

Uma perturbação escalar no espaço AdS deve obedecer à equação de KleinGordon, cuja solução é dada em termos de funções hipergeométricas. Impondo condições de contorno de Dirichlet no infinito espacial, chega-se a valores para os modos quasinormais com os mesmos valores dos polos dados pela equação (3.30). A mesma concordância é obtida para modos quasinormais de perturbações espinoriais e vetoriais. 



\section{Capítulo 4}

\section{Transições de fase de um sistema de um buraco negro RNAdS acoplado a um campo escalar carregado}

Em [40], Gubser sugere que, ao acoplar a gravidade com uma lagrangiana de Higgs, há uma quebra da simetria abeliana se existir um buraco negro carregado, e ocorre uma condensação de um campo escalar próximo ao horizonte de eventos. A referência [41] e trabalhos citados neste artigo explicam com detalhes o mecanismo da quebra de simetria, que envolve o efeito de uma massa efetiva cujo valor efetivo ao quadrado perto do horizonte de eventos é negativa, o que gera instabilidades no sistema.

Seja a lagrangiana como na seção 3.4

$$
16 \pi G_{N} \mathfrak{L}=R+\frac{6}{L^{2}}-\frac{1}{4} F_{\mu v} F^{\mu v}-\left|\nabla_{\mu} \psi-i q A_{\mu} \psi\right|^{2}-m^{2}|\psi|^{2}
$$

com a configuração

$$
\begin{aligned}
A_{\mu} \mathrm{d} x^{\mu} & =\Phi(r) \mathrm{d} t \\
\psi & =\psi(r) .
\end{aligned}
$$

Supomos que $\psi$ seja pequeno o suficiente para não interagir com os outros campos, i.e., que não haja retroação. ${ }^{1}$ Não levamos em conta um termo proporcional a $|\psi|^{4}$ porque (4.1) é a lagrangiana mais simples que exibe uma transição de fase.

\footnotetext{
${ }^{1}$ Retroação (ou backreaction em inglês) é o efeito do campo $\psi(r)$ na métrica que aparece através do tensor de energia-momento de $\psi$, quando calculamos o lado direito das equações de Einstein.
} 
A lagrangiana efetiva para o campo escalar é

$$
16 \pi G_{N} \mathfrak{L}_{\psi}=-g^{t t} q^{2} \Phi^{2}(r)|\psi|^{2}-g^{r r}\left|\nabla_{r} \psi\right|^{2}-m^{2}|\psi|^{2},
$$

de onde podemos definir uma massa efetiva como

$$
m_{\mathrm{eff}}^{2}=m^{2}+g^{00} q^{2} \Phi^{2}(r)
$$

Na presença de um horizonte de eventos, o termo $g^{00}$ é negativo fora do buraco negro e tende a $-\infty$ quando $r$ tende ao horizonte, denotado por $r_{H}$. Mesmo com a escolha de $\Phi\left(r_{H}\right)=0$, para valores de $r$ suficientemente próximos de $r_{H}, m_{\text {eff }}^{2}$ é negativo. De acordo com [40], por este motivo, há uma quebra de simetria e Gubser sugere que um buraco negro carregado pode exibir uma transição de fase para certos parâmetros do buraco negro e do campo escalar carregado. Utilizando a equivaência com uma teoria na borda, este fenômeno pode ser reinterpretado como uma supercondutividade na teoria holográfica.

\subsection{Modos marginalmente estáveis}

Modos marginalmente estáveis são definidos em [40] como uma solução independente do tempo ${ }^{2}$ da equação de movimento para $\psi$ obtida da lagrangiana (4.4) com módulo pequeno o suficiente para não retroagir com o espaço-tempo e atende às condições de contorno de ser regular no horizonte de eventos e tender a zero no infinito espacial.

Tomamos como métrica de fundo um buraco negro de Reissner-Norström assintoticamente anti-de Sitter, expressa por

$$
\mathrm{d} s^{2}=-f(r) \mathrm{d} t^{2}+\frac{\mathrm{d} r^{2}}{f(r)}+r^{2} \mathrm{~d} \Omega_{2, k}^{2}
$$

com $f(r)$ dado por

$$
f(r)=k-\frac{2 M}{r}+\frac{Q^{2}}{4 r^{2}}+\frac{r^{2}}{L^{2}}
$$

e $\mathrm{d} \Omega_{2, k}^{2}$ sendo a métrica de um espaço com curvatura constante de duas dimensões, expressa por

$$
\mathrm{d} \Omega_{2, k}^{2}=\left\{\begin{aligned}
\mathrm{d} \theta^{2}+\operatorname{sen}^{2} \theta \mathrm{d} \varphi^{2} & \text { se } k=1 \\
\mathrm{~d} \theta^{2}+\mathrm{d} \varphi^{2} & \text { se } k=0 \\
\mathrm{~d} \theta^{2}+\operatorname{senh}^{2} \theta \mathrm{d} \varphi^{2} & \text { se } k=-1
\end{aligned}\right.
$$

onde $2 k$ é o escalar de curvatura.

\footnotetext{
${ }^{2}$ Um modo marginalmente estável também pode ser entendido como uma solução com frequência nula de uma função que se comporta como $\psi(r, t)=e^{i \omega t} \psi(r)$
} 
Como desprezamos a retroação, a solução para $\Phi(r)$ é dada por

$$
\Phi(r)=\frac{Q}{r}-\frac{Q}{r_{H}},
$$

escolhendo a constante de integração tal que $\Phi(r)$ calculado no horizonte de eventos $r_{H}$ é igual a zero.

A equação de Euler-Lagrange para $\psi(r)$ obtida de (4.4) é

$$
-\frac{1}{\sqrt{-g}} \frac{\mathrm{d}}{\mathrm{d} r}\left(\sqrt{-g} g^{r r} \frac{\mathrm{d} \psi}{\mathrm{d} r}\right)+m_{e f f}^{2} \psi=0 .
$$

Fixando $Q=r_{H}=1,{ }^{3}$ podemos escrever a equação acima como

$$
f(r) \frac{\mathrm{d}^{2} \psi}{\mathrm{d} r^{2}}+\frac{1}{r^{2}}\left[-\frac{1}{4}+(2 r-1) k+\frac{\left(4 r^{3}-1\right)}{L^{2}}\right] \frac{\mathrm{d} \psi}{\mathrm{d} r}-m_{e f f}^{2} \psi=0
$$

com $f(r)$ agora expresso por

$$
f(r)=\frac{(r-1)}{r^{2}}\left[-\frac{1}{4}+k r+\frac{r\left(r^{2}+r+1\right)}{L^{2}}\right]
$$

e $m_{e f f}^{2}$ por

$$
m_{e f f}^{2}=m^{2}-\frac{4 q^{2}(r-1)}{-1+4 k r+\frac{4 r\left(r^{2}+r+1\right)}{L^{2}}} .
$$

Para que $f(r)$ não tenha uma raiz maior que $r_{H}=1$, os parâmetros $k$ e $L$ devem obedecer a

$$
-1+4 k+\frac{12}{L^{2}} \geq 0 \text {. }
$$

Se esta desigualdade não for satisfeita, a escolha $r_{H}=1$ está mal definida. Ao definir a suposta raiz maior que 1 , como $r_{H}=1$, o valor de $L$ é redefinido de modo que a desigualdade (4.14) seja válida. A temperatura do buraco negro é proporcional a $-1+4 k+\frac{12}{L^{2}}$. Podemos entender a desigualdade (4.14) como a afirmação de que a temperatura é positiva e a igualdade acontece quando $T=0$, que é inacessível de um estado com $T>0$ por um número finito de interações, como imposto pela terceira lei da termodinâmica.

Próximo de $r=r_{H}=1$, podemos expandir $\psi(r)$ como uma série de potências, cujos primeiros termos são dados por

$$
\psi(r)=1+\frac{4 m^{2}}{-1+4 k+\frac{12}{L^{2}}}(r-1)+O\left[(r-1)^{2}\right],
$$

\footnotetext{
${ }^{3}$ Como pode ser visto em [40], há certas simetrias de escala de interesse que justificam esta escolha. Em particular, é conveniente que as coordenadas sejam adimensionais ao implementar um método numérico.
} 
que nos sugere que condições devemos adotar para $\psi(r)$ e $\psi^{\prime}(1)$ e com isso integrar numericamente a equação (4.11). $\mathrm{O}$ valor $\psi\left(r_{H}\right)=1$ pode ser escolhido sem perda de generalidade.

A equação (4.11) foi resolvida pelo método de Runge-Kutta, primeiro com o objetivo de reproduzir os resultados de [40], e depois para calcular modos marginalmente estáveis para $k=-1$. Dadas as condições iniciais obtidas da equação (4.15) , calculamos os modos para vários parâmetros $L$ diferentes, mantendo os produtos $m^{2} L^{2}$ e $q L$ constantes ${ }^{4}$, e isolamos os modos que decaem para $r$ muito grande. As figuras (4.1) e (4.2) mostram os gráficos reproduzidos, com modos marginalmente estáveis não encontrados na referência [40].

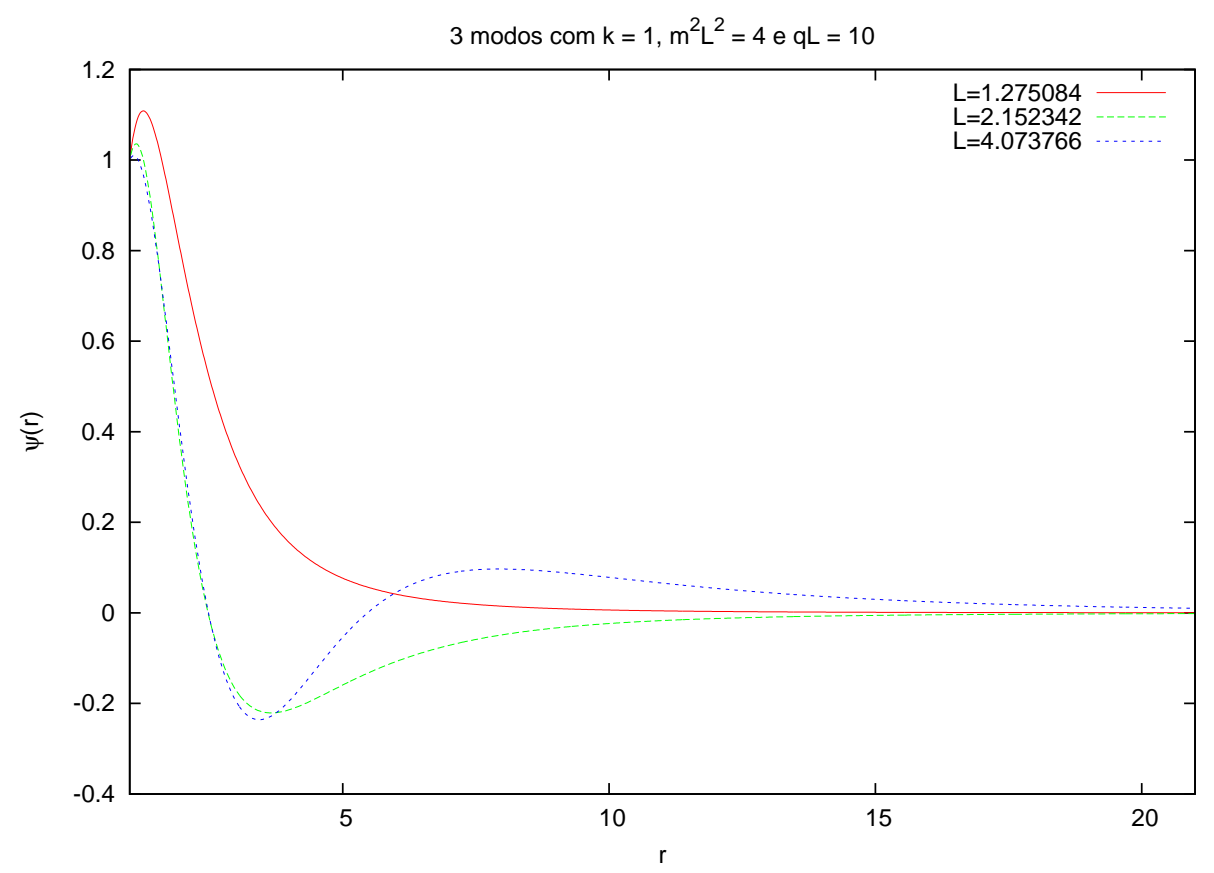

Figura 4.1: Modos reproduzidos e um modo adicional para $k=1$ e $m^{2}>0$.

Nota-se pela equação (4.11) que o comportamento de $\psi(r)$ para $r$ muito grande é o de uma reta. Sabendo o valor dos coeficientes calculados pelo método numérico, sabemos se $\psi(r)$ já exibe esse comportamento, que foi o critério utilizado para saber se $r$ é suficientemente grande para desprezar os outros termos da equação de movimento estudada. Dos modos calculados, escolhemos os que, após o $r$ ser suficientemente grande, exibem $|\psi(r)|<\varepsilon$, sendo $\varepsilon=10^{-5}$.

\footnotetext{
${ }^{4} \mathrm{~A}$ escolha de $m^{2} L^{2}$ e $q L$ constante é conveniente porque estes produtos são invariantes pelas simetrias de escala vistas em [40]
} 


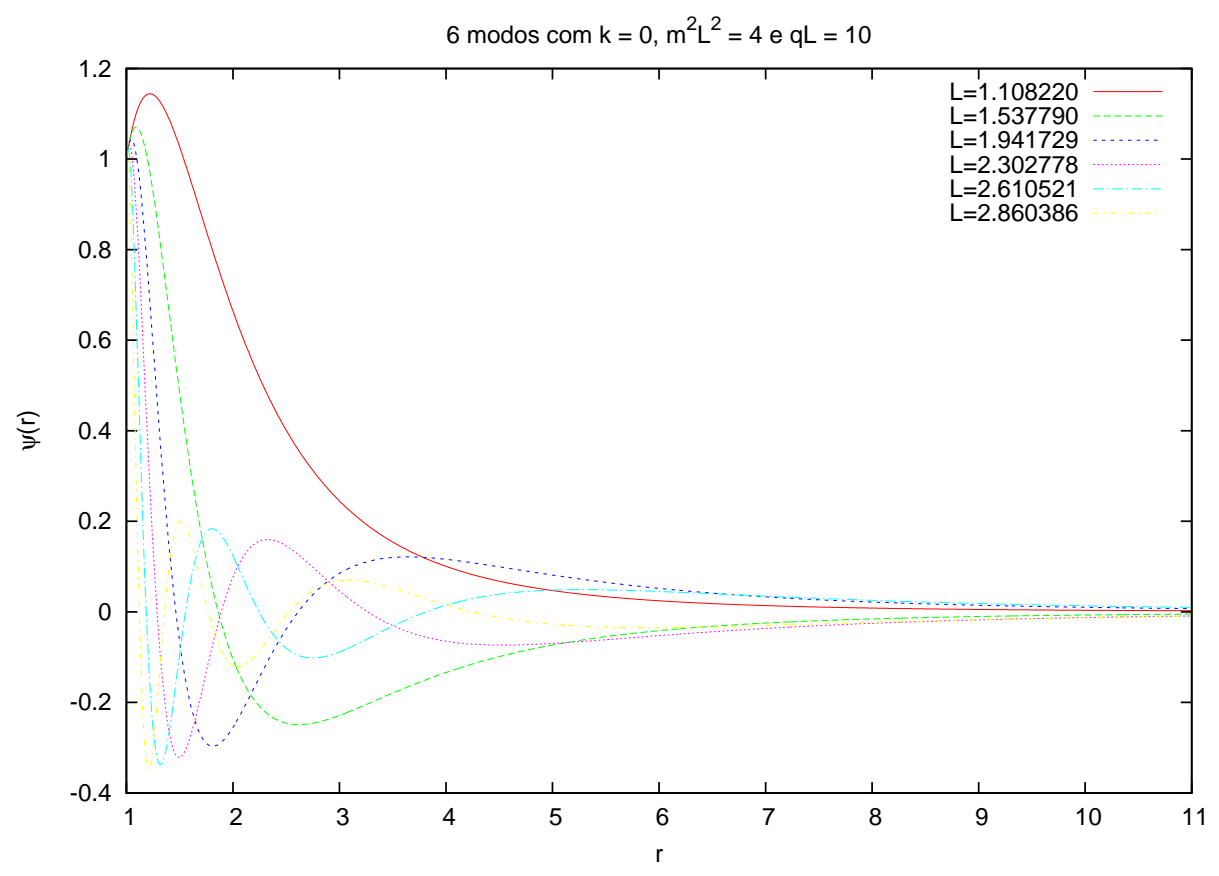

Figura 4.2: Modos previstos para $k=0$ e $m^{2}>0$.

Supondo que o valor de $\psi$ para $r$ grande seja uma função contínua do parâmetro $L$, e sabendo que $\psi\left(L_{a}, r\right)<0$ e $\psi\left(L_{b}, r\right)>0$ para $r$ grande, há um valor de $L$ entre $L_{a}$ e $L_{b}$ para o qual $\psi(L, r)=0$. Esses valores de $L$ foram obtidos pelo método da dicotomia (seção A.1.1), pois não temos outras informações além do valor de $\psi(L, r)$ para $r$ grande. Para várias configurações de $k$ e $m^{2} L^{2}$, montamos um gráfico do valor de $\Psi(r)$ para $r=1000$ em função de $L$, e para cada troca de sinal temos um modo marginalmente estável. Os modos marginalmente estáveis podem ser rotulados pelo número de vezes que mudam de sinal. Denotamos então estes modos por $L_{n}$, onde $n$ é o número de vezes que o gráfico dos modos marginalmente estáveis troca de sinal.

As figuras (4.3) e (4.4) são exemplos de modos encontrados por esse processo. Para o caso $k=0$, a referência [40] prevê um número infinito de modos concentrados no valor limite $L=\sqrt{12}$, como verificado, porém no caso $k=1$, encontramos um modo além dos já encontrados e vimos que o valor de $\Psi(r)$ para $r=1000$ diminui com $L$ com o comportamento de uma lei de potência, ou seja, sem mais mudanças de sinal. Para valores de $L$ ainda maiores, o sistema se comporta como uma perturbação escalar sem massa e carga em um espaço puramente AdS.

Para estudarmos o comportamento assintótico de (4.11), rearranjamos esta 


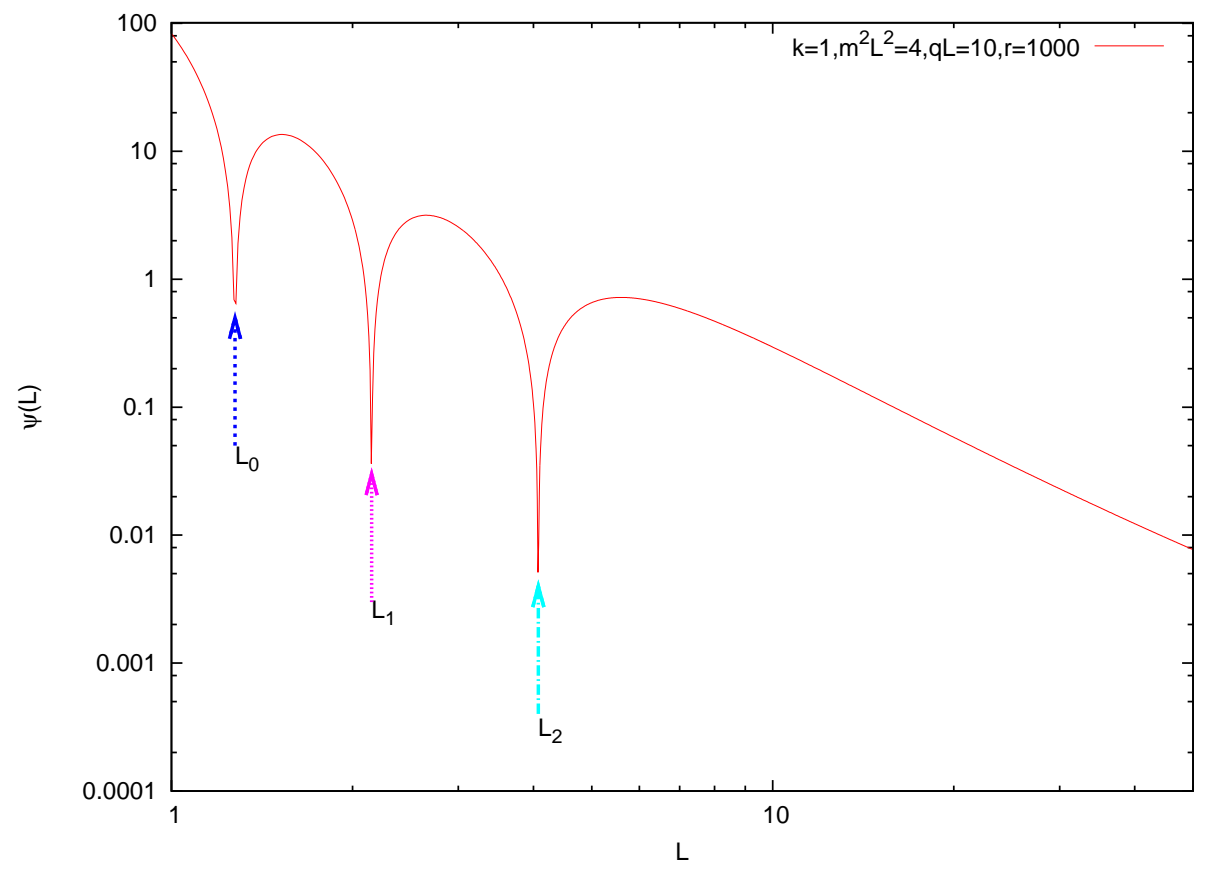

Figura 4.3: 3 modos marginalmente estáveis encontrados, seguido de um decaimento de lei de potência.

equação como

$$
\psi^{\prime \prime}+\frac{4}{r} \psi^{\prime}+\frac{L^{2}}{r^{2}}\left(k \psi^{\prime \prime}+m_{e f f}^{2} \psi\right)+\frac{L^{2}}{4 r^{3}}\left(\alpha \psi^{\prime \prime}+8 k \psi^{\prime}\right)+\frac{L^{2}}{4 r^{4}}\left(\psi^{\prime \prime}+\alpha \psi^{\prime}\right)=0
$$

com $\alpha=-1-4 k-4 / L^{2}$. Supondo um comportamento do tipo $\psi=A r^{-\Delta}, 5$ podemos considerar uma derivada como uma divisão por $r$, nesse caso, os termos restantes da equação acima resultam em

$$
\psi^{\prime \prime}+\frac{4}{r} \psi^{\prime}+\frac{m^{2} L^{2}}{r^{2}} \psi=0
$$

Substituindo $\psi=A r^{-\Delta}$ na equação acima vemos que $\Delta$ deve obedecer a relação

$$
\Delta_{ \pm}=\frac{3 \pm \sqrt{9+4 m^{2} L^{2}}}{2}
$$

o que permite escrever o comportamento assintótico de $\psi$ como

$$
\psi(r) \simeq \frac{\psi^{(+)}}{r^{\Delta_{+}}}+\frac{\psi^{(-)}}{r^{\Delta_{-}}}
$$

\footnotetext{
${ }^{5}$ Esta suposição é basicamente uma aplicação do método de Frobenius.
} 


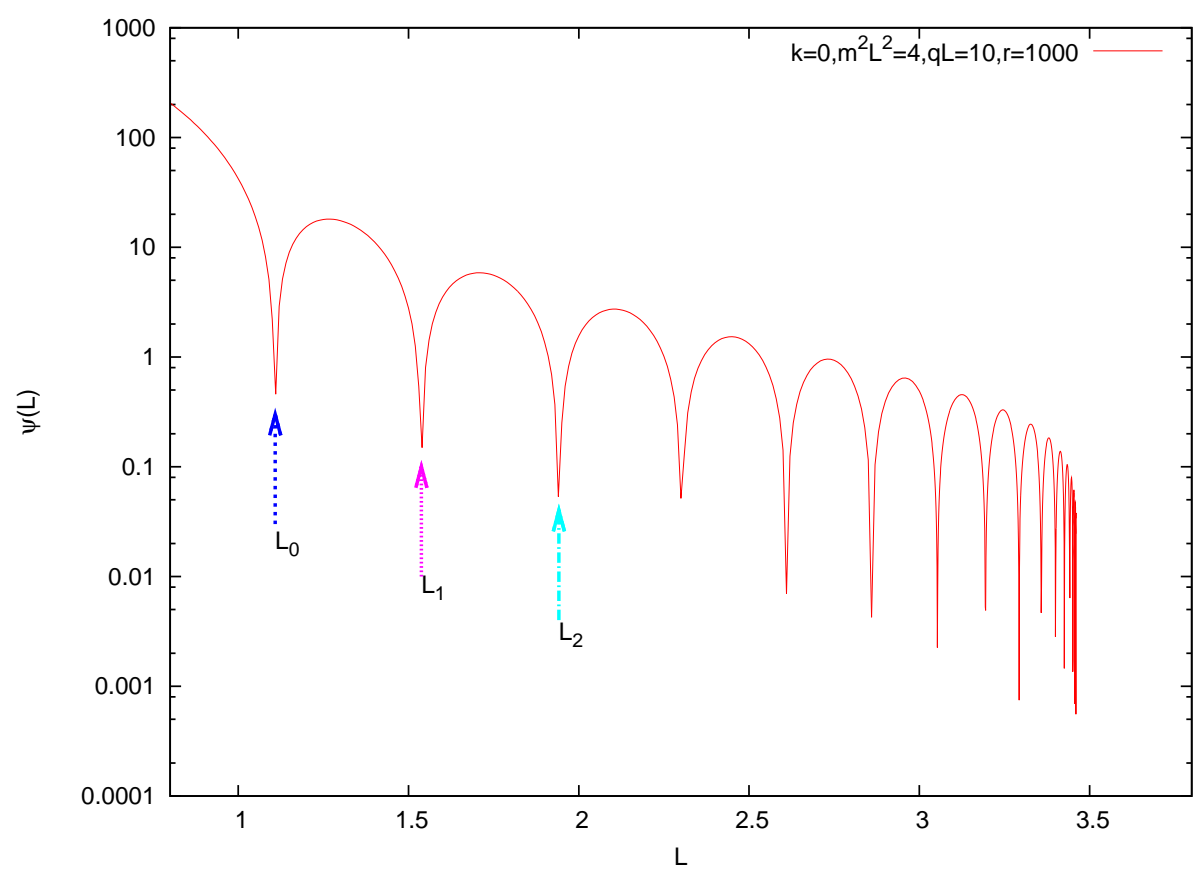

Figura 4.4: Modos marginalmente estáveis encontrados como previsto.

Segundo a correspondência AdS/CFT, como pode ser visto na seção 3.4, os termos $\psi^{( \pm)}$estão ligados a um operador $O_{ \pm}$na teoria dual. O valor esperado deste operador é um parâmetro de ordem que é nulo para temperaturas maiores que uma certa temperatura crítica. Esta temperatura crítica é dada pelos parâmetros do primeiro modo marginalmente estável, que é o menor valor de $L$ para o qual há uma solução não trivial das equações de movimento de um supercondutor holográfico (equações (3.19) e (3.20)). Interpretando modos marginalmente estáveis como soluções da equação de movimento dependente do tempo para o campo $\psi(r, t)$ com frequência nula, acreditamos que os modos marginalmente estáveis sejam uma fronteira entre modos quasinormais estáveis e instáveis.

\subsection{Modos Quasinormais}

Para tentar responder algumas questões deixadas em aberto em [40], tratamos a equação de movimento obtida da lagrangiana sem supor dependência apenas em $r$. A equação de movimento para $\psi$ é então igual à equação de um modo quasinormal que obtemos ao fazer uma perturbação escalar em um buraco negro Reissner-Nordström-anti-de Sitter. Supondo que exista uma transição entre 
um modo quasinormal instável e um modo quasinormal estável, a mudança deve ocorrer em um conjunto de parâmetros iguais aos de um modo marginalmente estável se a parte real da frequência também for nula.

Um segundo interesse em estudar os modos quasinormais vem da interpretação de Horowitz e Hubeny mencionada na seção 3.5 que diz que a frequência imaginária de um modo quasinormal está associada à escala de tempo de retorno ao equilírio térmico na teoria de campo dual dado pela correspondência AdS/CFT. Imaginamos então que a supercondutividade deve estar relacionada com modos quasinormais instáveis. Comparando com os modos marginalmente estáveis, esperamos que os modos quasinormais instáveis estejam numa região com temperaturas mais baixas.

Partindo da lagrangiana

$$
16 \pi G_{N} \mathfrak{L}=R+\frac{6}{L^{2}}-\frac{1}{4} F_{\mu \nu}^{2}-\left|\nabla_{\mu} \Psi-i q A_{\mu} \Psi\right|^{2}-m^{2}|\Psi|^{2}
$$

obtemos a equação de movimento

$$
\frac{1}{\sqrt{-g}} \partial_{\mu}\left(\sqrt{-g} g^{\mu v} \partial_{v}\right) \Psi-2 i q g^{\mu v} A_{\mu} \partial_{\nu} \Psi-q^{2} g^{\mu v} A_{\mu} A_{v} \Psi-m^{2} \Psi=0 .
$$

Supondo novamente que

$$
\begin{aligned}
A_{\mu} \mathrm{d} x^{\mu} & =\Phi(r) \mathrm{d} t, \\
\Phi(r) & =\frac{Q}{r}-\frac{Q}{r_{H}},
\end{aligned}
$$

ainda sem retroação, mas o campo $\Psi$ não depende apenas de $r$. Após a separação de variáveis

$$
\Psi=\frac{\psi(r, t)}{r} Y_{l}^{m}(\theta, \varphi)
$$

temos

$$
\begin{gathered}
-\frac{\partial^{2} \Psi}{\partial t^{2}}+f(r) \frac{\partial}{\partial r}\left(f(r) \frac{\partial \Psi}{\partial r}\right)+2 i q \Phi(r) \frac{\partial \Psi}{\partial t} \\
-\left[\frac{f^{\prime}(r) f(r)}{r}+\frac{l(l+1) f(r)}{r^{2}}+m^{2} f(r)-q^{2} \Phi^{2}(r)\right] \Psi=0,
\end{gathered}
$$

onde

$$
f(r)=\frac{r-1}{4 r^{2}}\left(-1+4 k r+\frac{4 r\left(r^{2}+r+1\right)}{L^{2}}\right)
$$

se escolhermos $Q=r_{H}=1$.

Definindo uma coordenada tartaruga por

$$
x(r)=\int_{\infty}^{r} \frac{1}{f\left(r^{\prime}\right)} \mathrm{d} r^{\prime}
$$


temos

$$
x(r)=\sum_{i=1}^{4} \frac{r_{i}^{2}}{D_{i}^{2}} \log \left(r-r_{i}\right)
$$

onde $r_{i}$ são os zeros de $f(r)$ e $D_{i}=\left.\frac{\mathrm{d}}{\mathrm{d} r}\left(r^{2} f(r)\right)\right|_{r=r_{i}}$.

Duas raízes de $f(r)$ formam um par de complexos conjugados, mas a expressão para $x(r)$ é real. Denotando as raízes complexas por $r_{R} \pm i r_{I}$ e as reais por $r_{1}=1$ e $r_{2}$, escrevemos a coordenada tartaruga como

$x(r)=\frac{1}{D_{1}} \log (r-1)+\frac{r_{2}^{2}}{D_{2}} \log \left(r-r_{2}\right)+\alpha \log \left[\left(r-r_{R}\right)^{2}+r_{I}^{2}\right]+2 \beta \arctan \left(\frac{r_{I}}{r-r_{R}}\right)$

com

$$
\begin{gathered}
\alpha=\frac{\left(r_{R}^{2}-r_{I}^{2}\right) D_{R}+2 r_{R} r_{I} D_{I}}{D_{R}^{2}+D_{I}^{2}}, \\
\beta=\frac{2 r_{R} r_{I} D_{R}-\left(r_{R}^{2}-r_{I}^{2}\right) D_{I}}{D_{R}^{2}+D_{I}^{2}}, \\
D_{R}+i D_{I}=\left.\frac{\mathrm{d}}{\mathrm{d} r}\left(r^{2} f(r)\right)\right|_{r=r_{R}+i r_{I}} .
\end{gathered}
$$

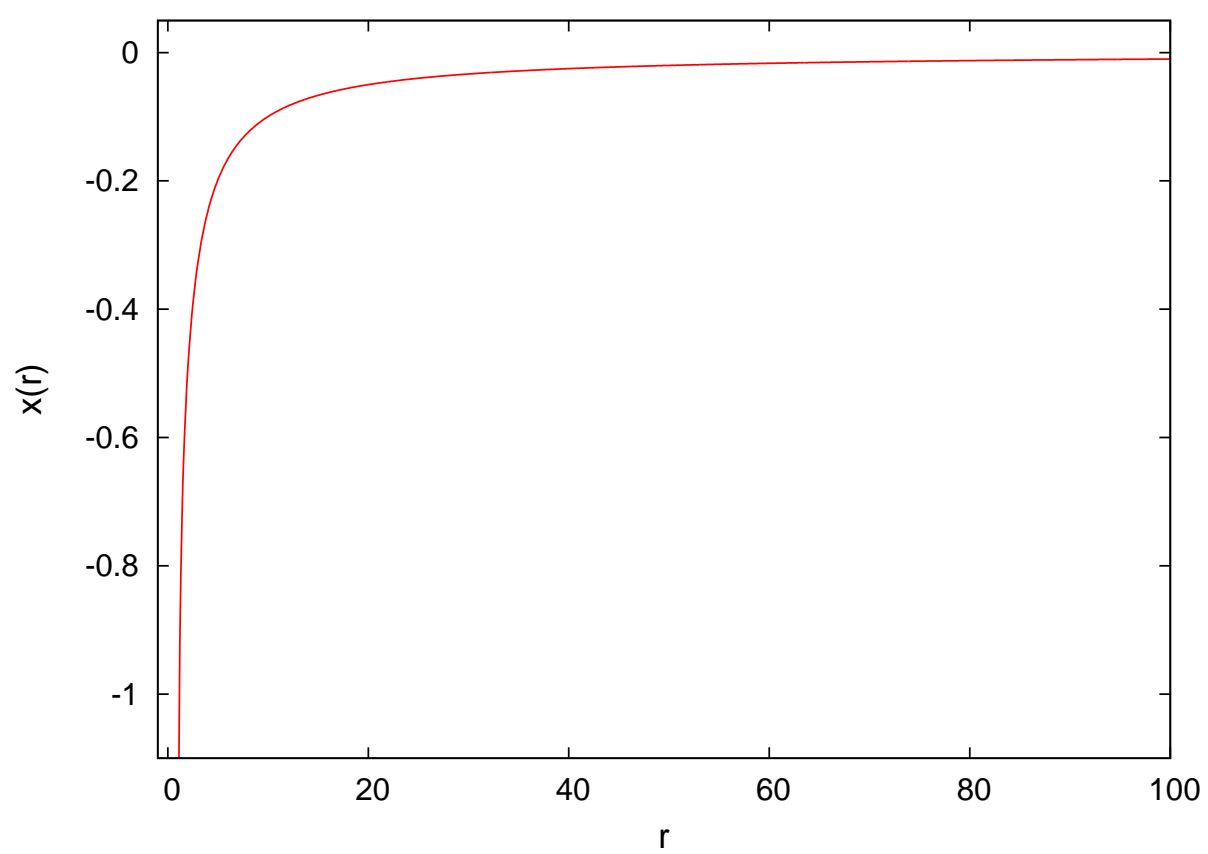

Figura 4.5: Gráfico da coordenada tartaruga com $k=1, L=1, Q=1$ e $r_{H}=1$. 
A coordenada $x(r)$ mapeia o intervalo $(1, \infty)$ em $(-\infty, 0)$, e seu gráfico pode ser visto na figura 4.5. Seria conveniente uma expressão analítica para a inversa $r(x)$ da coordenada tartaruga. A inversa existe, mas para ser calculada é necessário um método numérico de busca de solução. Dado um $\bar{x}<0$, calculamos a solução para $x(r)-\bar{x}=0$ pelo método da dicotomia (seção A.1.1) O método de Newton (seção A.1.2) não é indicado por ter uma vizinhança de convergência muito pequena.

No novo sistema de coordenadas, escrevemos a equação (4.25) como

$$
-\frac{\partial^{2} \psi}{\partial t^{2}}+2 i q \Phi(r) \frac{\partial \psi}{\partial t}+\frac{\partial^{2} \psi}{\partial x^{2}}-V(r) \psi=0
$$

com

$$
V(r)=f(r)\left[\frac{f^{\prime}(r)}{r}+\frac{l(l+1)}{r^{2}}+m^{2}\right]-q^{2} \Phi^{2}(r),
$$

lembrando que $r$ é uma função de $x$ calculada pela inversa de (4.29).

Não conhecemos uma solução analítica de (4.33), então encontramos uma solução numérica para $\psi(x, t)$ utilizando o método de diferenças finitas (seção A.4.1). Definindo uma grade em $(x, t)$ por

$$
\begin{aligned}
& x=-j \times \Delta x, \\
& t=+l \times \Delta t,
\end{aligned}
$$

rotulamos o valor de $\psi(x, t)$ por $\psi_{j, l}=\psi(-j \times \Delta x, l \times \Delta t)$. Usando o método de diferena̧s finitas definido na seção A.4.1, aproximamos a equação (4.33) por

$$
\begin{aligned}
& -\frac{\psi_{j, l+1}-2 \psi_{j, l}+\psi_{j, l-1}}{\Delta t^{2}}+i 2 q \Phi(r) \frac{\psi_{j, l+1}-\psi_{j, l-1}}{2 \Delta t} \\
& +\frac{\psi_{j+1, l}-2 \psi_{j, l}+\psi_{j-1, l}}{\Delta x^{2}}-V(r) \psi_{j, l}=0 .
\end{aligned}
$$

Isolando o termo $\psi_{j, l+1}$, obtemos

$$
\begin{aligned}
& \psi_{j, l+1}=-\frac{(1+i q \Phi \Delta t)^{2}}{1+q^{2} \Phi^{2} \Delta t^{2}} \psi_{j, l-1}+\frac{1+i q \Phi \Delta t}{1+q^{2} \Phi^{2} \Delta t^{2}} \times \\
& \left\{\left[2-2 \frac{\Delta t^{2}}{\Delta x^{2}}-\Delta t^{2} V(r)\right] \psi_{j, l}+\frac{\Delta t^{2}}{\Delta x^{2}}\left(\psi_{j+1, l}+\psi_{j-1, l}\right)\right\} .
\end{aligned}
$$

Como condição inicial, utilizamos $\psi(x, 0)$ igual a uma gaussiana e $\psi(x, 0)=0$. Os parâmetros da gaussiana não são importantes no comportamento dos modos quasinormais. Escolhemos $\psi(x, 0)=10^{-3} e^{(x+5)^{2}}$.

O critério de estabilidade de von Neumann (seção A.4.3), possui algumas dificuldades mencionadas na seção 2.6 devido ao fato do potencial crescer com $r^{2}$ 
quando $r$ tende ao infinito. Verificamos no entanto que o método é estável para todos os parâmetros escolhidos se $\frac{\Delta t}{\Delta x} \leq \frac{1}{2}$, então escolhemos $\frac{1}{2}$ como razão entre as distâncias entre os pontos da grade em todas as contas numéricas.

Tentamos aplicar o método de Horowitz-Hubeny (seção 2.5) para conferir os valores de frequências quasinormais obtidas pelo método de diferenças finitas. Ao escrever a equação de movimento com a separação $\psi\left(x^{\mu}\right)=\frac{Z(r)}{r} e^{-i \omega v} Y_{l m}(\theta, \varphi) \mathrm{e}$ a mudança de variáveis $x=\frac{1}{r}$, obtemos

$$
s(x) \frac{\mathrm{d}^{2} Z}{\mathrm{~d} x^{2}}+\frac{t(x)}{\left(x-x_{+}\right)} \frac{\mathrm{d} Z}{\mathrm{~d} x}+\frac{u(x)}{\left(x-x_{+}\right)^{2}} Z=0,
$$

onde as funções $s(x), t(x)$ e $u(x)$ são polinômios dados por

$$
\begin{aligned}
& s(x)=x^{2} A_{0}, \\
& t(x)=\left\{2\left(x-x_{+}\right) x A_{0}+x A_{1}+2 i x^{2}\left[\omega+q\left(x-x_{+}\right)\right]\right\}, \\
& u(x)=\left(x-x_{+}\right)\left[A_{1}-m^{2}+2 i q\left(x-x_{+}\right) x\right],
\end{aligned}
$$

com

$$
\begin{aligned}
& A_{0}=\left[\frac{x^{3}}{4}-\frac{x^{2}}{x_{+}}-\frac{x^{2}+x_{+} x+x_{+}^{2}}{L^{2} x_{+}^{3}}\right] \\
& A_{1}=\left[-\frac{x^{3}}{x_{+}}+\frac{\left(2 x-x_{+}\right) x^{3}}{4}-\frac{x^{3}+2 x_{+}^{3}}{L^{2} x_{+}^{3}}\right],
\end{aligned}
$$

onde definimos $l=0$ e $Q=1$.

Como visto na seção 2.5, expandimos $Z(x)$ como uma série de potências e obtemos um polinômio de grau infinito em $\omega$. As frequências são encontradas truncando este polinômio em um grau $N$ e à medida que aumentamos $N$ vemos se as raízes convergem para algum valor. Como os coeficientes do polinômio são complexos, utilizamos o método de Jenkins-Traub (seção A.1.6).

\subsection{Transições de fase}

Os potenciais exibidos nas figuras 4.6 e 4.7 mostram, para $L>1$, uma barreira de potencial próximo ao horizonte de eventos, uma mudança de sinal e finalmente uma divergência para $+\infty$ quando $r$ tende ao infinito. Como a equação de movimento possui um termo proporcional a $\frac{\partial \psi}{\partial t}$, não é necessariamente verdade que um potencial positivo definido implica em uma estabilidade, no entanto, os sistemas estudados não foram exceção a esta regra. Presumimos que o primeiro modo 


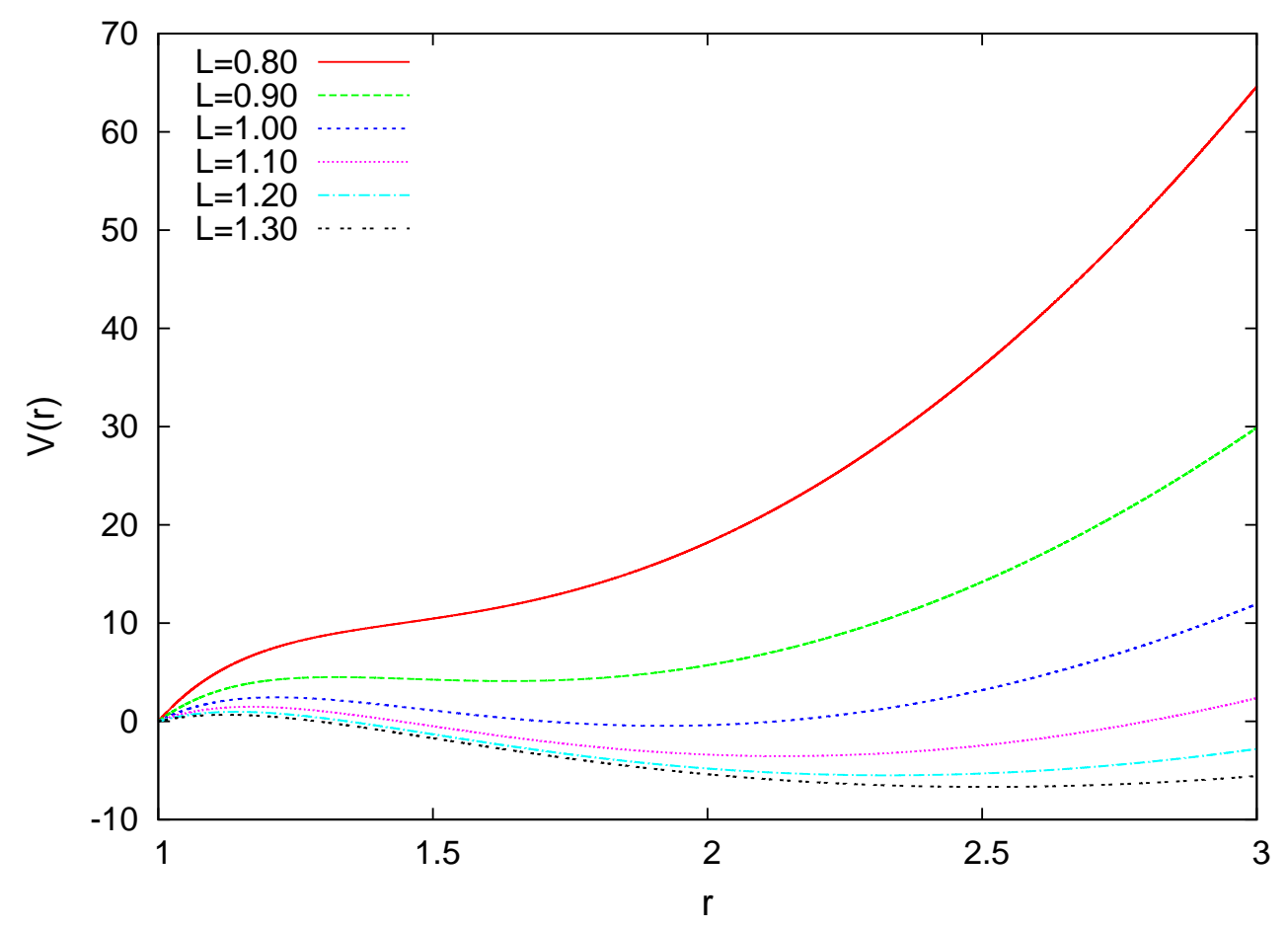

Figura 4.6: Potenciais efetivos em função de $r$ para diferentes valores de $L$.

instável esteja relacionado com o primeiro valor de $L$ cujo potencial admite um estado ligado com energia negativa [42].

Na figura 4.8 mostramos o comportamento de cinco modos diferentes calculados, um deles sendo o mais próximo obtido da mudança de estabilidade nos valores atribuídos a $L$. Neste gráfico, os valores dos produtos $m^{2} L^{2}=4$ e $q L=10$ são mantidos constantes e variamos o parâmetro $L$. Observamos uma mudança de estabilidade em um valor de $L$ próximo a 1.27 , que é próximo ao primeiro modo marginalmente estável em [40].

Ajustamos o comportamento dos modos como uma função do tipo $\psi\left(r_{*}, t\right)=A \exp \left(\omega_{i} t\right) \cos \left(\omega_{r} t+\delta\right)$. Este ajuste não é capaz de dizer o sinal de $\omega_{r}$, e os valores calculados mostram uma descontinuidade na primeira derivada. Supondo que o comportamento de $\omega_{r}$ seja uma função suave do parâmetro $L$, essa descontinuidade indica uma mudança de sinal. Escolhemos então o sinal de $\omega_{r}$ como o mesmo de $\omega_{i}$ para montar a figuras (4.9) e (4.10).

Observando o comportamento para valores de $L>2$, encontramos um modo secundário. Este modo, assim como o modo principal, inverte a estabilidade em um valor próximo a um modo marginalmente estável visto em [40], mas o valor de $L$ é próximo ao segundo modo marginaomente estável. O sistema como um 


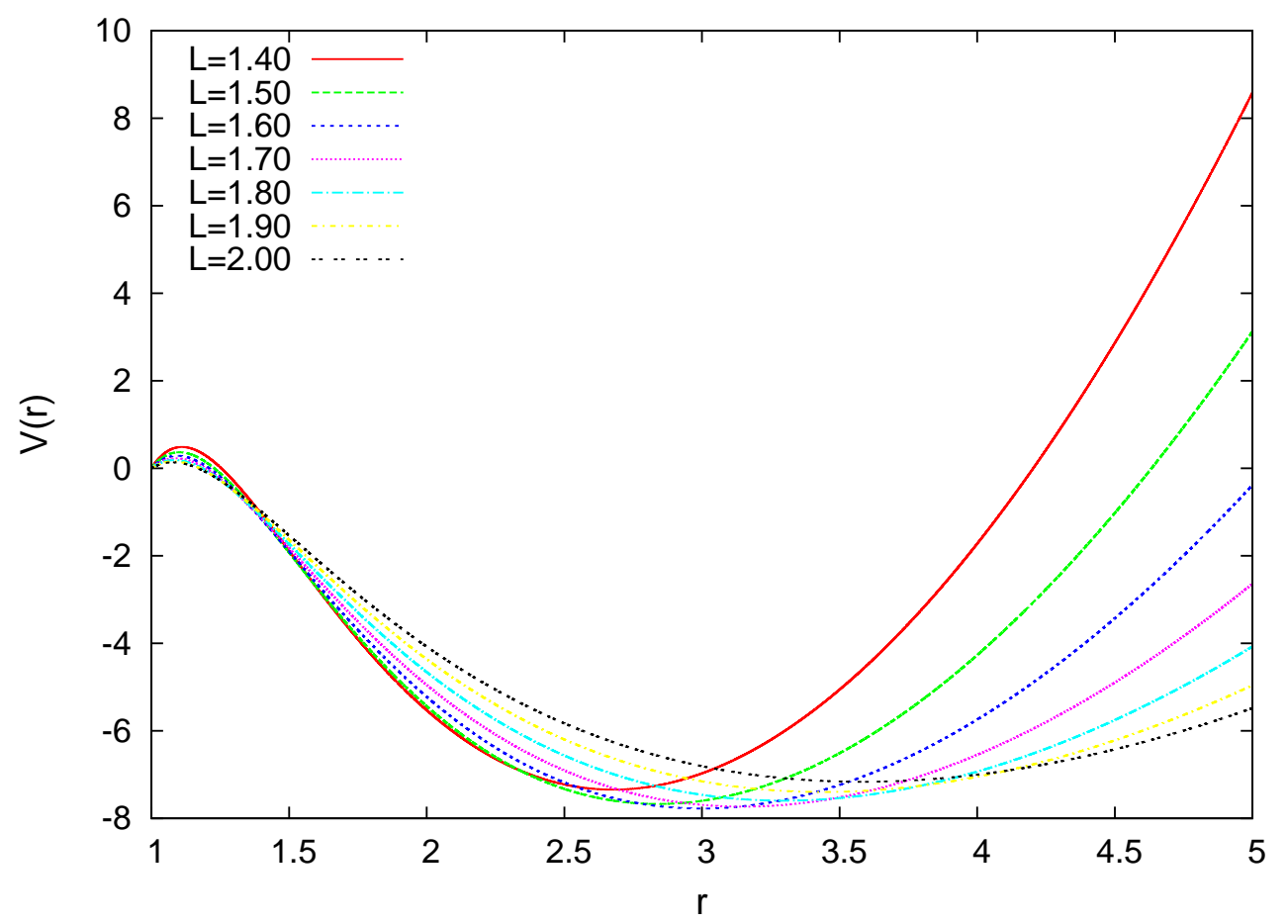

Figura 4.7: Potenciais efetivos em função de $r$ para diferentes valores de $L$.

todo, exibido na figura 4.11, não inverte a estabilidade devido ao primeiro modo permanecer instável enquanto o segundo modo muda de estabilidade (figura 4.12).

Novamente ajustamos o comportamento do segundo modo como uma oscilação de frequência constante e escolhemos o sinal de $\omega_{r}$ como o mesmo de $\omega_{i}$. Os resultados podem ser visto na figura (4.13). Os valores que obtivemos para a mudança de sinal é de fato um pouco menor que os modos marginalmente estáveis comparados. Acreditamos que esta diferença se deva ao fato de métodos numéricos usados em equações diferenciais parciais possuírem erros maiores que métodos usados em equações diferenciais ordinárias.

Nas figuras 4.14 e 4.15 comparamos o resultado obtido pelo método de Horowitz-Hubeny com os valores obtidos anteriormente. A linha vertical separa a região estável da instável. Na região estável, o comportamento da parte real é semelhante, enquanto a parte imaginária difere por uma escala, mas com um comportamento qualitativamente parecido. No entanto, ao se aproximar do valor do primeiro modo marginalmente estável, o comportamento obtido pelo método Horowitz-Hubeny não se aproxima de zero como esperado e como obtido pelo método de diferenças finitas.

Em [16], os autores discutem que o método não consegue calcular frequências 


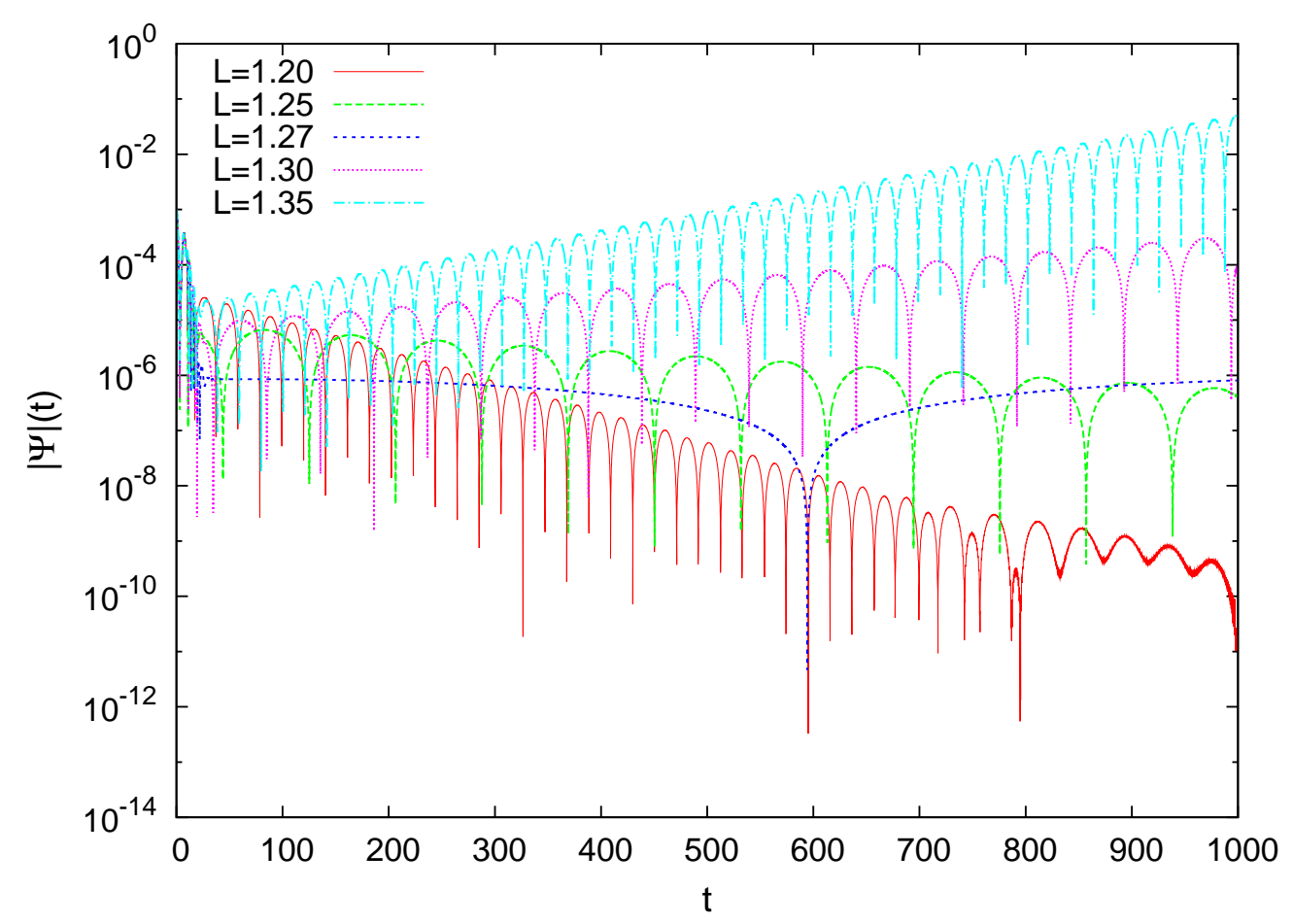

Figura 4.8: Modos quasinormais em função de $t$.

próximas a zero. Outro problema enfrentado é que o resultado obtido na região instável não parece convergir quando aumentamos a ordem $N$ em que truncamos o polinômio. Observamos também que o módulo dos termos dos polinômios possuem ordens de grandezas muito diferentes um dos outros, e estes valores são muito maiores do que seriam se a carga $q$ do campo escalar fosse nula. Com um $N$ grande, o polinômio em questão começa a apresentar os problemas mencionados na seção A.1.7. Como os resultados do método de diferenças finitas concorda com os valores obtidos em [40], tomamos estes valores como corretos.

A temperatura Hawking do buraco negro é dada por

$$
T=\frac{1}{4 \pi}\left(\frac{4 k r_{H}^{2}-Q^{2}}{4 r_{H}^{3}}+\frac{3 r_{H}}{L^{2}}\right),
$$

que na escolha de $k=Q=r_{H}=1$ pode ser escrita como

$$
T=\frac{1}{16 \pi}\left(3+\frac{12}{L^{2}}\right) .
$$

Nas figuras 4.16, 4.17 e 4.18 mostramos os valores das frequências do primeiro e do segundo modo em função da temperatura Hawking. Como esperado, 


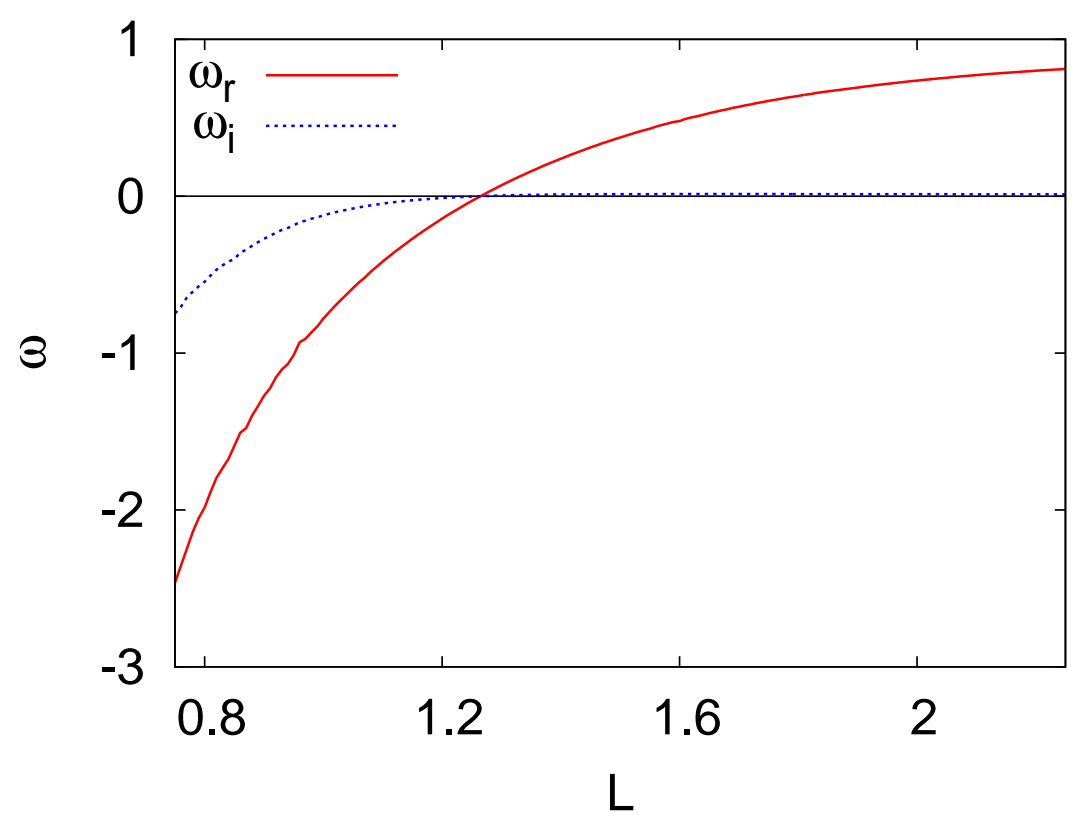

Figura 4.9: Frequências do modo principal.

a fase instável do sistema está localizada em uma região com temperatura menor que uma temperatura crítica. Concluímos então que o modelo apresentado exibe uma transição de fase em uma temperatura $T_{c} \simeq 0.21$, com os valores utilizados e em unidades em que o horizonte de eventos vale 1. Segundo [16], a parte imaginária da frequência dos modos quasinormais estão relacionados à escala de tempo de retorno ao equilíbrio de uma perturbação do estado térmico do sistema. Um sistema com modos quasinormais estáveis deve então estar relacionado com um sistema térmico sem condensação, então acreditamos que esta mudança de estabilidade esteja relacionada a uma transição de fase de um supercondutor na teoria holográfica.

A mesma mudança de estabilidade é observada em buracos negros com horizontes de eventos que exibem outras topologias $(k=0$ ou $k=-1$ na equação (4.26)), assim como em perturbações com $m^{2}<0$. Em todos os casos observados, a mudança de estabilidade dos modos quasinormais ocorre em um valor de $L$ próximo ao primeiro modo marginalmente estável, consistente com o caso $k=1, m^{2} L^{2}=4$ e $q L=10$ estudado em detalhes neste trabalho. 


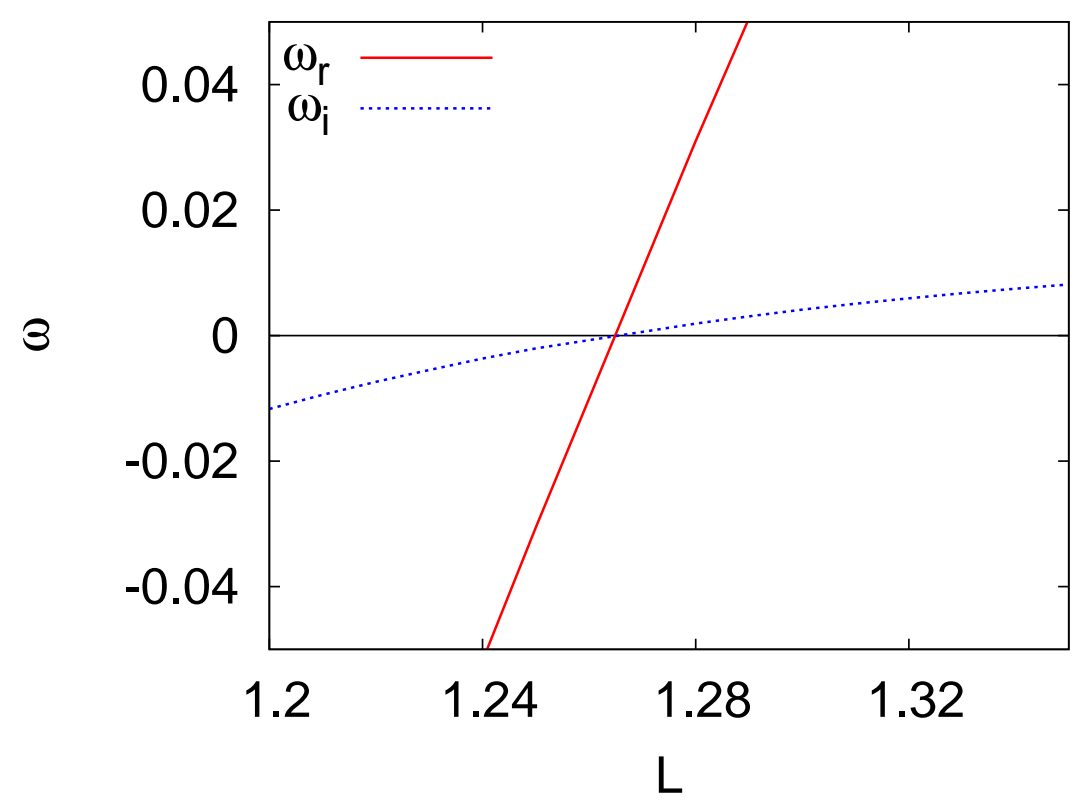

Figura 4.10: Frequências do modo principal próximas à mudança de estabilidade.

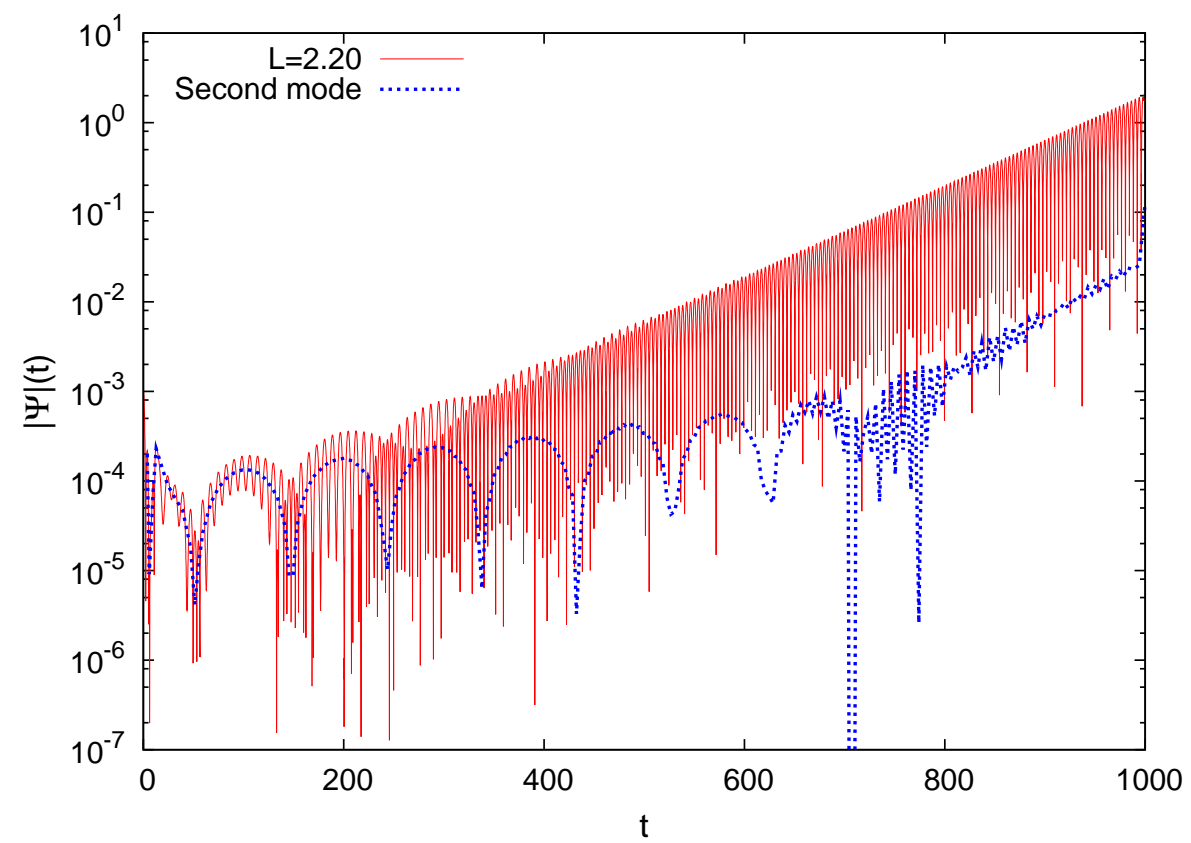

Figura 4.11: Comportamento do sistema e do segundo modo em função de $t$. 


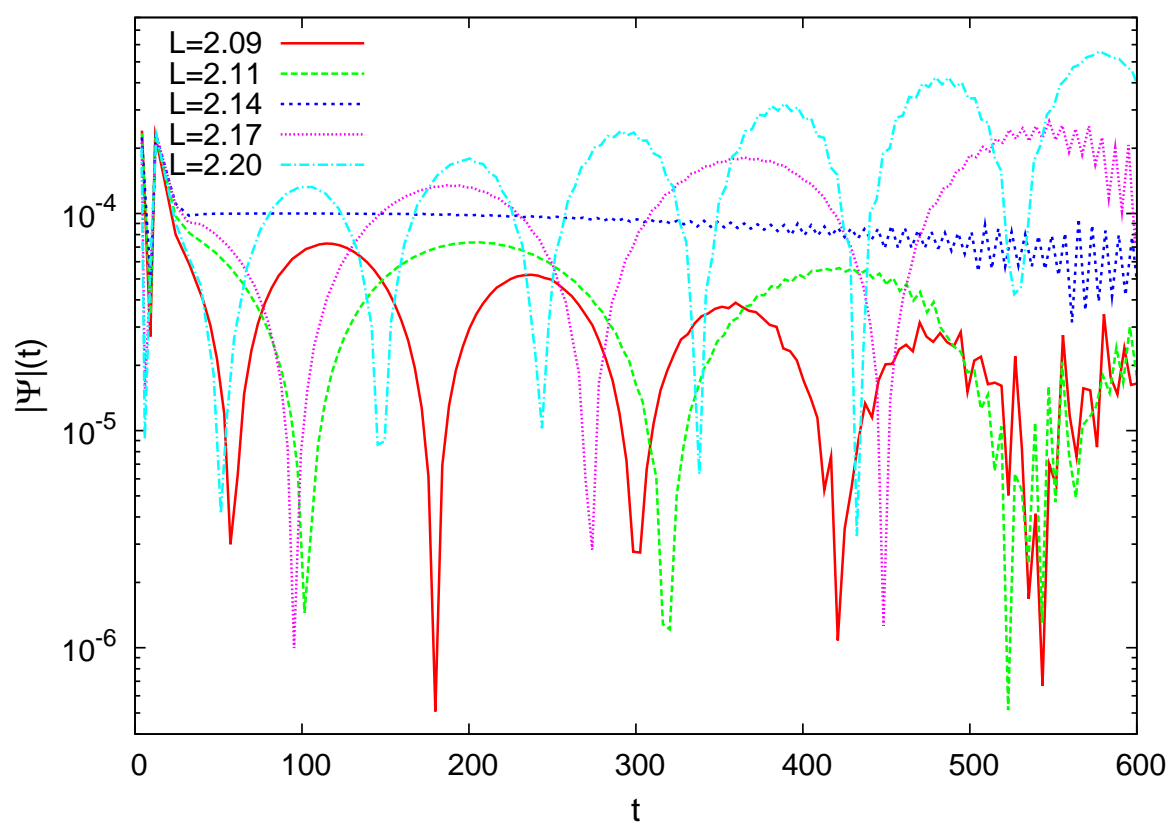

Figura 4.12: Comportamento do segundo modo em função de $t$.

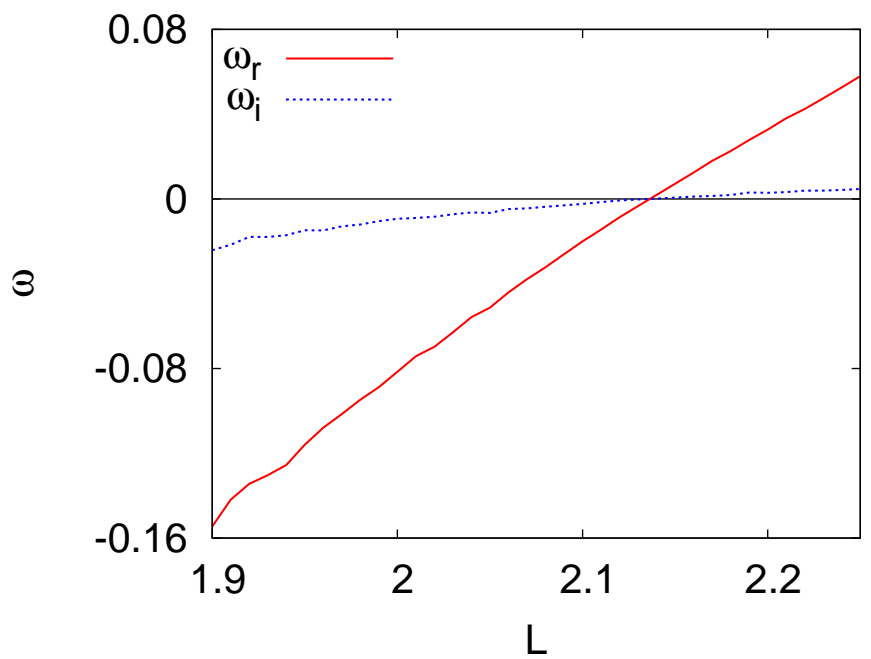

Figura 4.13: Frequências do segundo modo. 


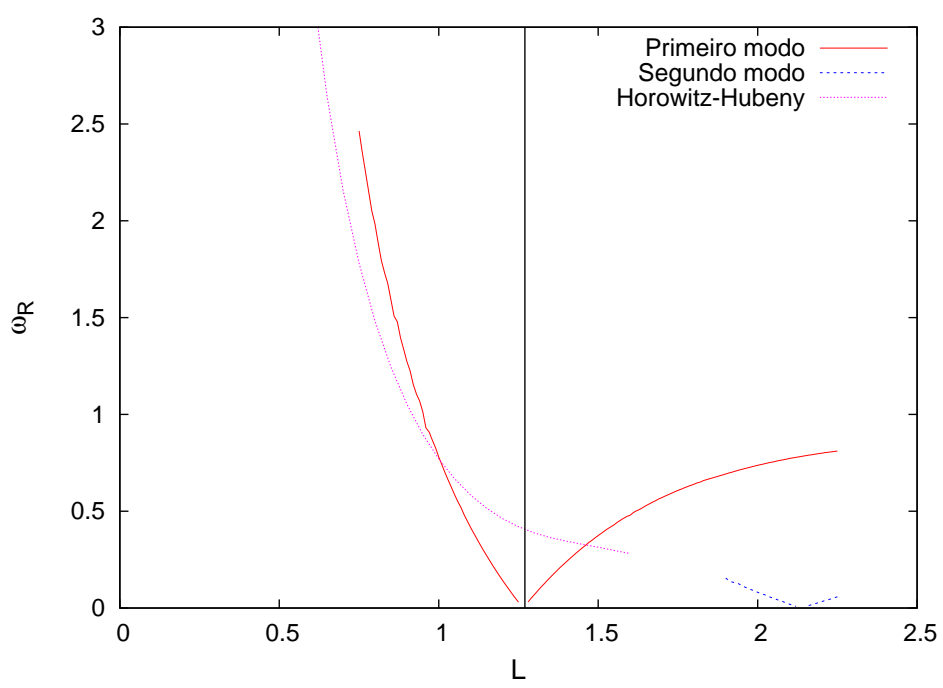

Figura 4.14: Comparação dos valores da parte real da frequência obtidos por integração numérica e pelo método de Horowitz-Hubeny.

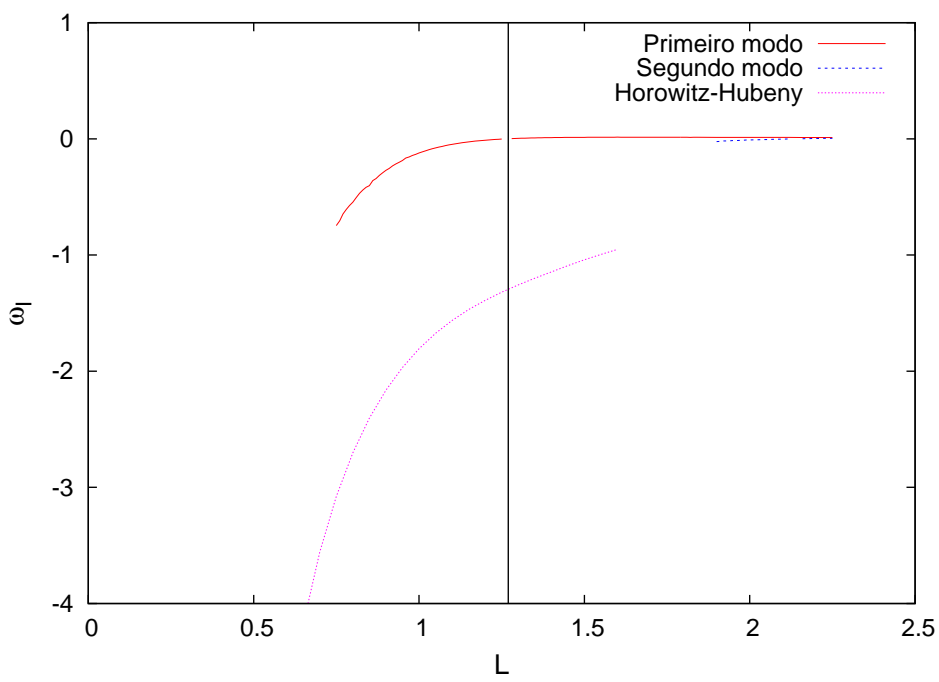

Figura 4.15: Comparação dos valores da parte imaginária de frequência obtidos por integração numérica e pelo método de Horowitz-Hubeny. 


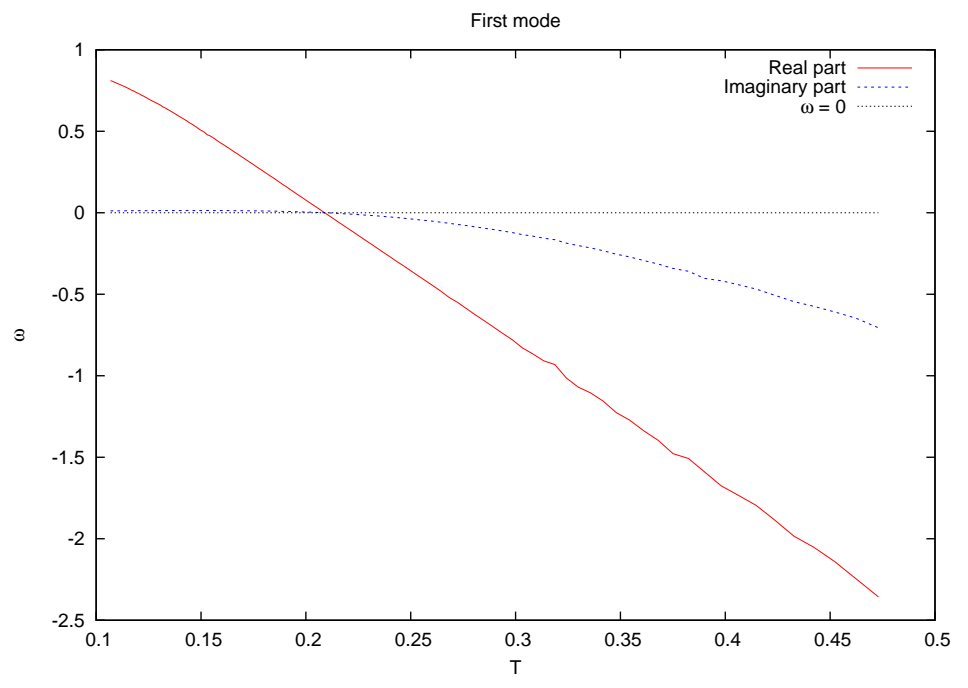

Figura 4.16: Frequências do modo principal em função da temperatura.

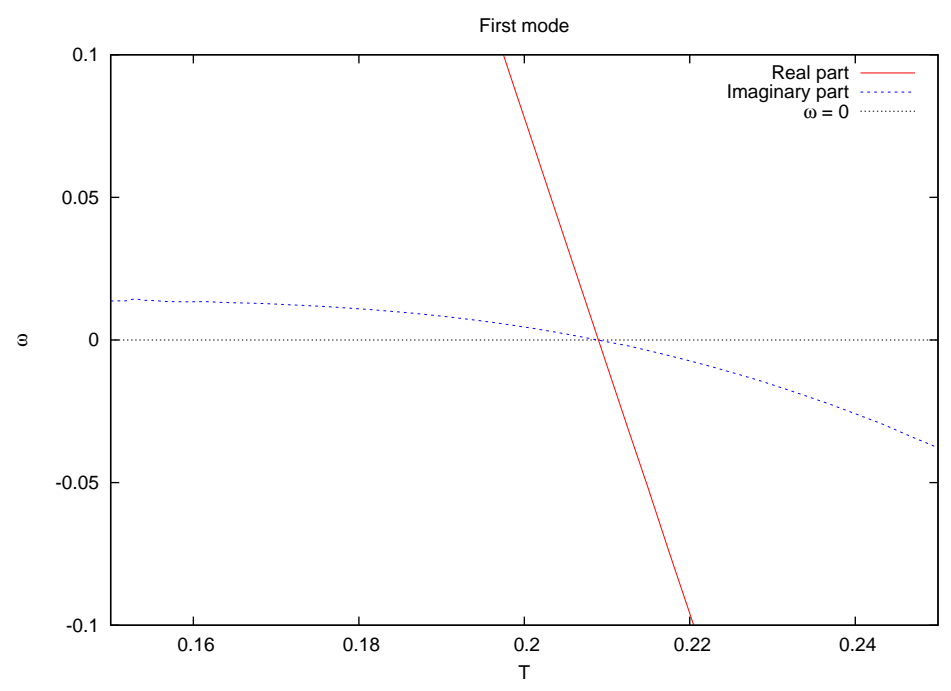

Figura 4.17: Frequências do modo principal em função da temperatura próximas à mudança de estabilidade. 


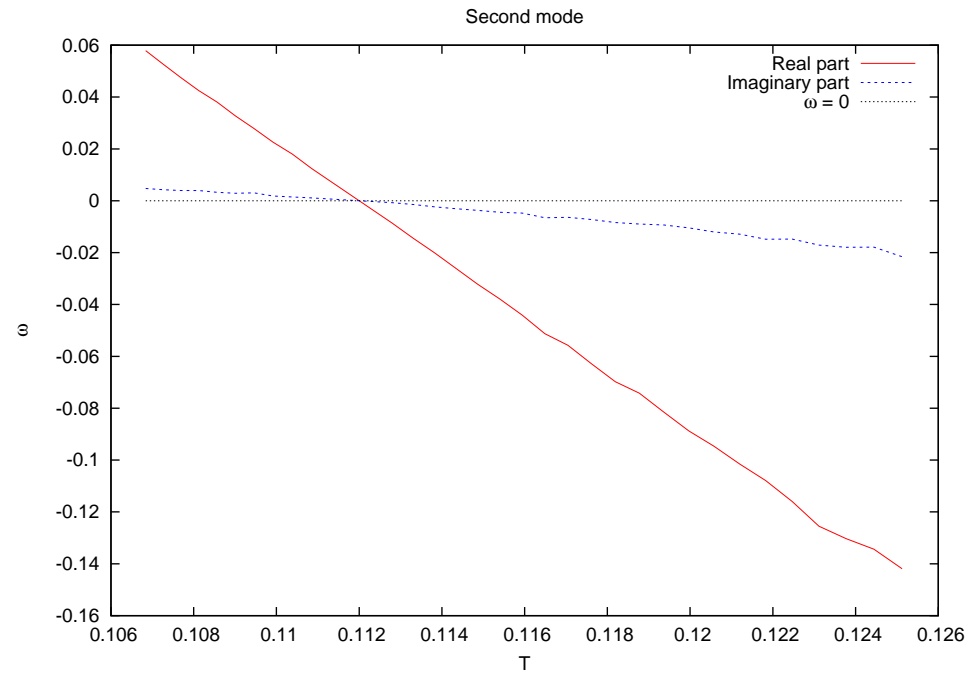

Figura 4.18: Frequências do segundo modo em função da temperatura.

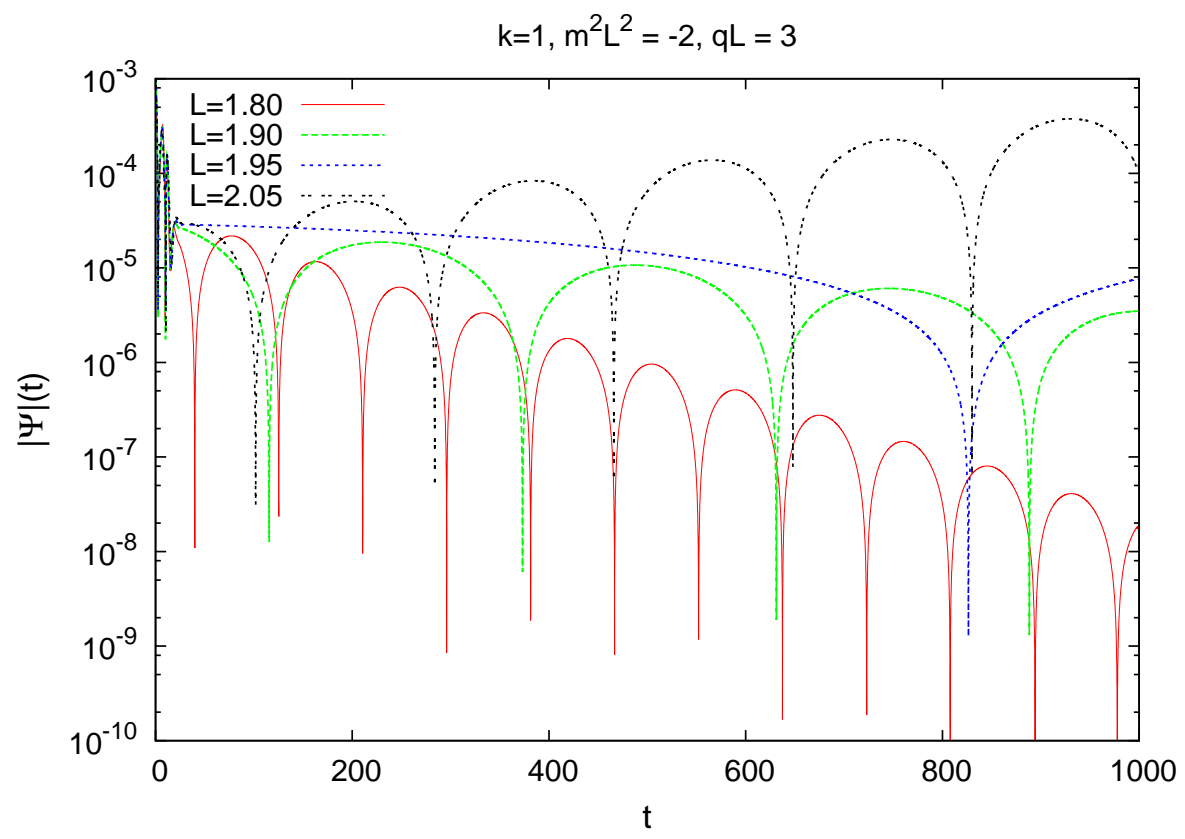

Figura 4.19: Modos quasinormais estáveis e instáveis. 


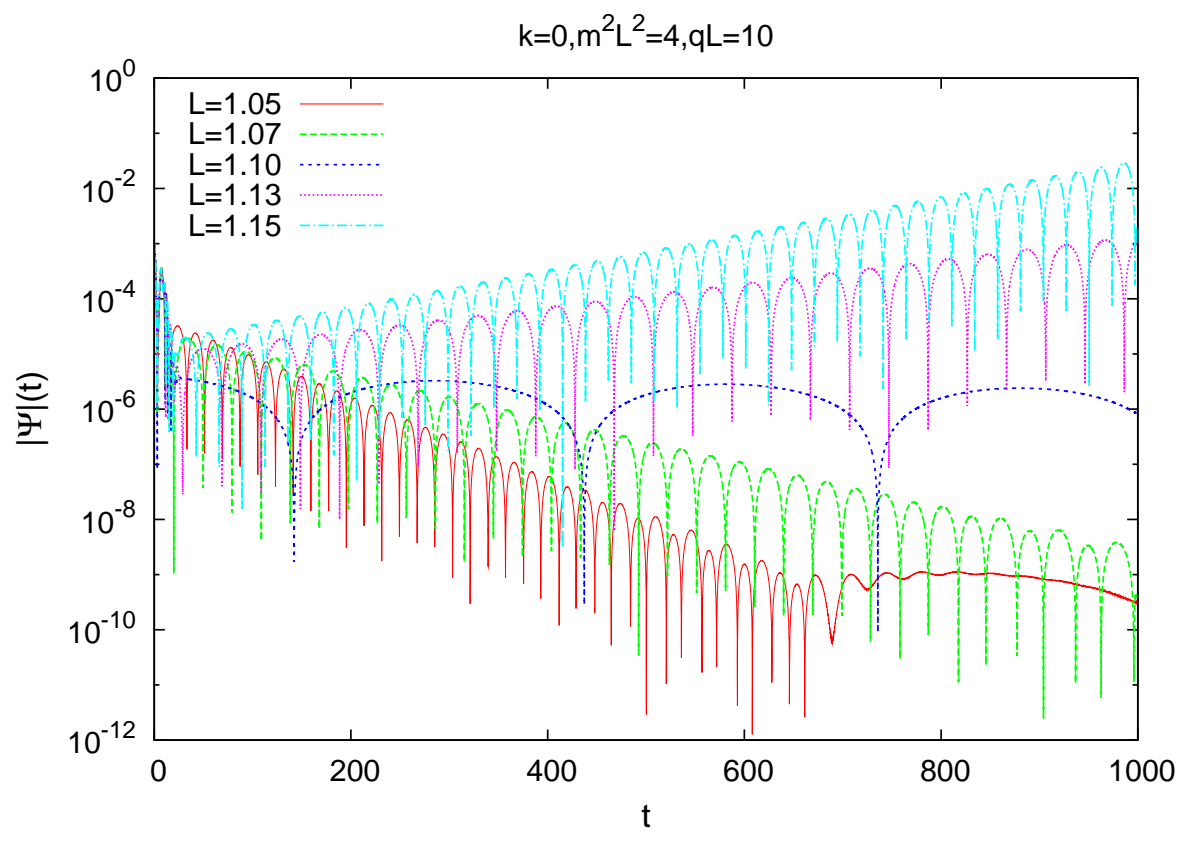

Figura 4.20: Modos quasinormais estáveis e instáveis.

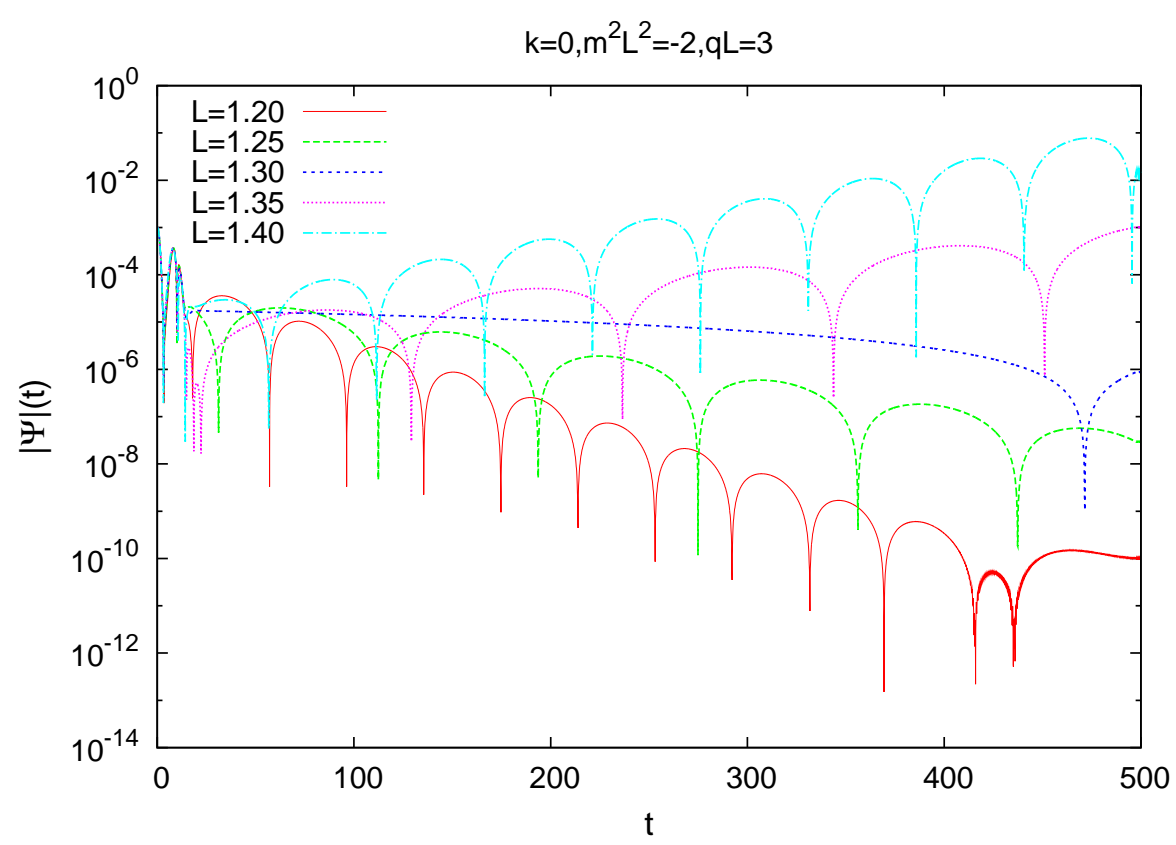

Figura 4.21: Modos quasinormais estáveis e instáveis. 


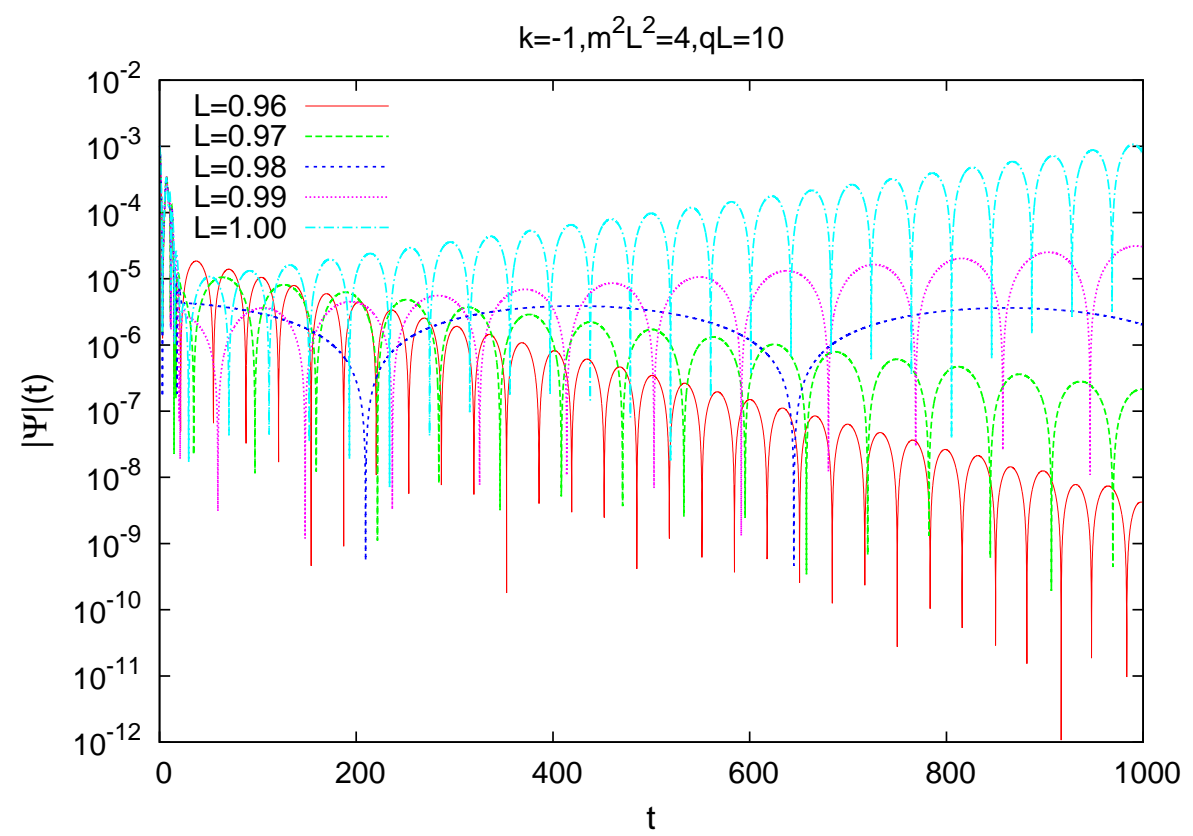

Figura 4.22: Modos quasinormais estáveis e instáveis.

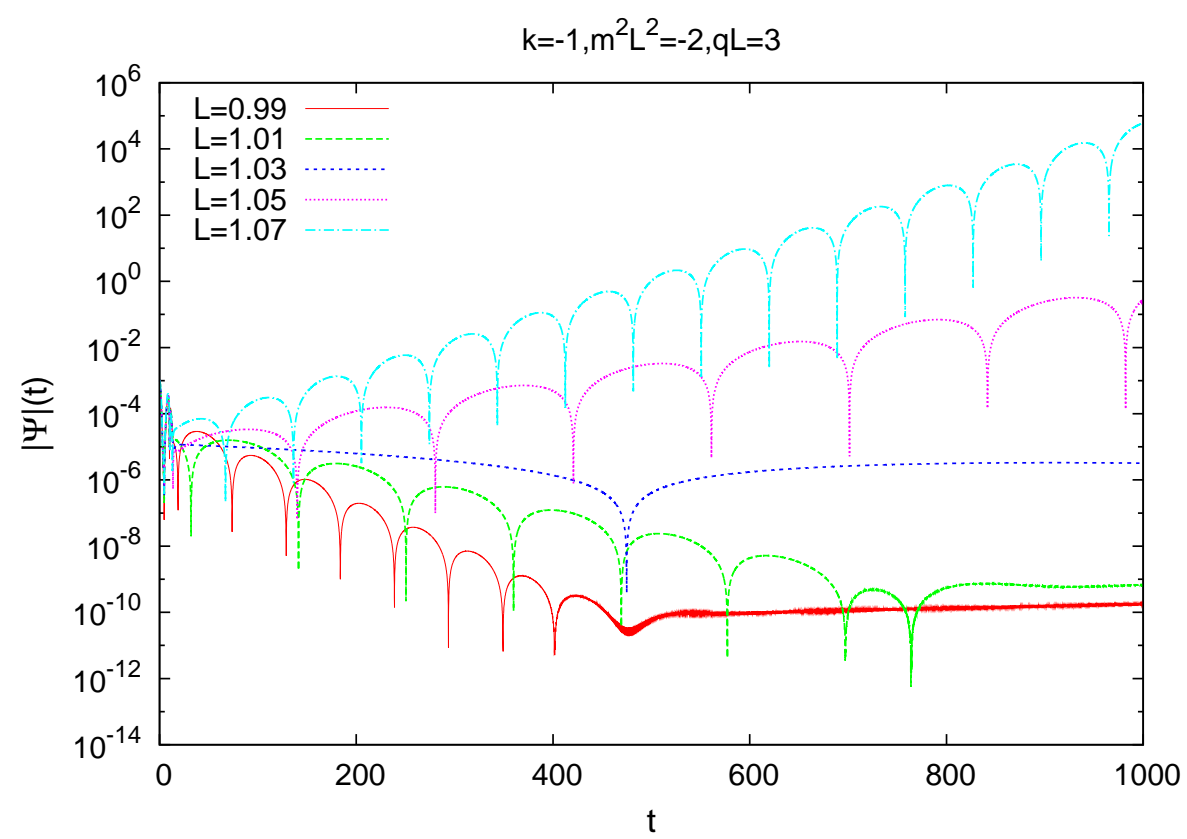

Figura 4.23: Modos quasinormais estáveis e instáveis. 


\section{Capítulo 5}

\section{Discussões finais}

Escrevemos este trabalho com o objetivo de encontrar alguma relação entre modos quasinormais e alguma interpretação em uma teoria dual segundo a correpondência AdS/CFT. O cálculo de modos quasinormais em várias soluções de buracos negros conhecidas não é novidade (vários exemplos são dados em [10; 9]), mas o papel destes modos na correspondência AdS/CFT ainda é um tema pouco estudado.

O tema escolhido foi resolver as equações de movimento de uma lagrangiana da matéria acoplada à lagrangiana de Einstein-Hilbert, que possui a mesma equação que uma perturbação escalar de um modo quasinormal. A solução destas equações nos dá então informações da estabilidade do sistema de um buraco negro descrito por uma métrica e uma partícula descrita por um campo escalar.

Como métrica de fundo, escolhemos um buraco negro de Reissner-Nordtröm assintoticamente anti-de Sitter motivados pela referência [40], que encontra uma quebra de simetria e a compara com propriedades de um supercondutor holográfico vistas na seção 3.4. Nosso intuito foi aplicar as ferramentas desenvolvidas no projeto de pesquisa para testar a estabilidade dos modos quasinormais no contexto dado em [40].

Fizemos um uso intenso de métodos numéricos para integrar as equações de movimento encontradas neste trabalho, assim como cálculos secundários, como calcular a inversa de uma coordenada tartaruga. Algumas particularidades do problema impedem o uso de métodos mais sofisticados, mas o objetivo principal de trabalhar com métodos rudimentares como visto no capítulo A é que eles podem ser aplicados em outros trabalhos mais complicados.

Calculando a solução $\psi(x, t)$ da equação de movimento (4.33), encontramos a mudança de estabilidade prevista em [40] com uma boa concordância quantitativa, considerando erros numéricos inerentes aos métodos. O resultado mais interessante deste trabalho foi dar uma interpretação física aos modos marginalmente estáveis. Vimos que eles estão de fato ligados a uma mudança de estabilidade, 
como mostrado pelo comportamento dos modos quasinormais, mas a existência de mais de um modo marginalmente estável não implica em mais de uma fronteira entre estabilidade e instabilidade do sistema, apenas em uma superposição de diferentes modos quasinormais e para cada um deles há uma mudança de estabilidade em um valor diferente da temperatura do buraco negro. A única fronteira física é de uma região em que todos os modos quasinormais são estáveis para uma outra em que um dos modos é instável, que é o suficiente para que o sistema seja instável.

Pretendemos em um trabalho futuro desenvolver um método numérico para calcular os parâmetros de ordem $\psi^{( \pm)}$do supercondutor holográfico sem comparar séries de potências em limites diferentes. Uma outra possível continuação deste trabalho é considerar termos nas equações de movimento que foram desprezados, como a retroação, ou trabalhar com menos suposições, como simetria esférica do espaço-tempo, pois os métodos numéricos estudados não dependem de tais simplificações.

Os métodos estudados neste trabalho também foram utilizados no estudo de estabilidade do modelo Hořava-Witten dependente de tempo [43]. Nesta teoria, podemos modelar o Big Bang como o resultado de uma colisão de branas. Ao estudar o comportamento de perturbações escalares, vimos que antes da colisão, o modelo não apresenta instabilidades e as frequências dependem fortemente dos parâmetros do modelo se a perturbação for massiva. Próximo do colisão, a parte real das frequências quasinormais cresce indefinidamente e surge uma instabilidade, mas não sabemos se o sistema físico é instável ou se esta instabilidade vem do método numérico.

Estudamos também em [44] a estabilidade de buracos negros com simetria de Lifshitz em New Massive Gravity [45], que descreve uma gravidade massiva em 3 dimensões covariante e que preserva paridade para partículas de spin 2. Implementamos o método de diferenças finitas para calcular perturbações escalares e espinoriais e verificamos que estas perturbações são estáveis se os parâmetros do buraco negro e das perturbações são tais que o potencial efetivo que aparece na equação de perturbação é positivo definido, como previsto pelo critério de estabilidade mencionado na seção 2.3 .

Por fim, investigamos em [46] o efeito de um modelo de matéria escura interagente na rotação de galáxias e no desalinhamento entre distribuições de matéria escura e matéria bariônica. Modelando estar distrubuições como barras rígidas, que por sua vez podem ser modeladas por um sistema de 2 corpos, verificamos que uma distribuição inicial de desalinhamento se espalha, de acordo com as observações [47]. Implementamos também um algoritmo genético que calcula qual intensidade de força do modelo interagente ajusta melhor as observações. 


\section{Apêndice A}

\section{Métodos numéricos}

Métodos numéricos são, muitas vezes, a única alternativa conhecida para encontrar a solução de um problema específico. Muitos problemas não possuem solução analítica, isto é, a solução não pode ser escrita em termos das funções elementares (polinomial, trigonométrica, exponencial, etc.) e, portanto, o estudo de métodos numéricos é tão importante quanto o estudo de métodos desenvolvidos em Física-Matemática.

Muitos métodos apresentados nesse capítulo são extremamente básicos e é de fato contraditório supor conhecimento de teoria quântica de campos em outros capítulos e não supor conhecimento de métodos numéricos, mas esta é a realidade observada em vários alunos de pós-graduação trabalhando em gravitação, que imaginamos ser o público alvo desta tese.

\section{A.1 Método de busca de soluções}

\section{A.1.1 Método da dicotomia}

Seja $f(x)$ uma função contínua no intervalo $[a, b]$ e $f(a) \cdot f(b)<0$. Existe um ponto $c \in(a, b)$ tal que $f(c)=0$. Dados $a$ e $b$ iniciais com $f(a) \cdot f(b)<0$, calculamos $c$ a partir do seguinte algoritmo:

1. Calcular $f(a)$ e $f(b)$;

2. Calcular $c=\frac{a+b}{2}$;

3. Calcular $f(c)$;

4. Se $f(a) \cdot f(c)>0$, a solução pertence ao intervalo $(c, b)$, defino $a=c$, caso contrário, defino $b=c$ e volto para o passo 1 . 
Na prática, não calculamos de fato o valor de $c$, apenas reduzimos o intervalo ao qual o valor desejado pertence. Como cada passo reduz o tamanho do intervalo a metade do anterior, depois de um número $n$ de repetições, tomamos como solução o ponto médio do intervalo final com um erro $\varepsilon=\frac{b-a}{2^{n}}$. Então para achar uma solução com um erro $\varepsilon$ desejado, deve-se repetir os passos acima $n$ vezes com $n>\log _{2}\left(\frac{b-a}{\varepsilon}\right)$.

A vantagem deste método é que só precisamos saber calcular $f(x)$, o que é particularmente útil se o cálculo da derivada for muito trabalhoso. Este método não é capaz de encontrar uma raiz com multiplicidade par. Se houver uma descontinuidade no intervalo $(a, b)$ pode ocorrer uma mudança de sinal sem uma solução no intervalo, e o algoritmo vai tender ao valor em que a função é descontínua. Se houver mais de uma solução em $(a, b)$, não há um critério claro para saber para qual solução o algoritmo vai encontrar, neste caso deve-se utilizar o algoritmo com algum conhecimento prévio de em que região cada raiz se encontra.

\section{A.1.2 Método de Newton-Raphson}

Para uma certa tentativa se solução $x_{i}$, calcula-se a próxima tentativa calculando a reta tangente a $f(x)$ passando pelo ponto $\left(x_{i}, f\left(x_{i}\right)\right)$ e tomo como próxima tentativa o ponto em que essa reta cruza o eixo $x$.

A reta tangente é dada por

$$
r(x)=f\left(x_{i}\right)+f^{\prime}\left(x_{i}\right)\left(x-x_{i}\right),
$$

definindo $x_{i+1}$ tal que $r\left(x_{i+1}\right)=0$, temos

$$
x_{i+1}=x_{i}-\frac{f\left(x_{i}\right)}{f^{\prime}\left(x_{i}\right)} .
$$

Se existe $\bar{x}$ tal que $f(\bar{x})=0, f(x)$ e $f^{\prime}(x)$ forem contínuas e $f^{\prime}(x) \neq 0$, existe uma vizinhança de $\bar{x}$ em que, para qualquer escolha de tentativa inicial $x_{0}$, a sequência $x_{i}$ converge para $\bar{x}$. Se $f^{\prime \prime}(\bar{x})$ for uma função real, a convergência é quadrática. Se $f^{(3)}$ for real e limitado na vizinhança de convergência e $f^{\prime}(\bar{x})$ e $f^{\prime \prime}(\bar{x}) \neq 0$, o erro de cada iteração é dado por

$$
\left|\varepsilon_{i+1}\right|=\frac{1}{2} \frac{f^{\prime \prime}(\bar{x})}{f^{\prime}(\bar{x})}\left(\varepsilon_{i}\right)^{2}+O\left[\left(\varepsilon_{i}\right)^{3}\right]
$$

onde $x_{i}=\bar{x}+\varepsilon_{i}$. Se $f^{\prime}(\bar{x})=0$, a convergência é apenas linear.

Em algumas funções a vizinhança de convergência pode ser pequena ou não existir. Por exemplo, para $f(x)=x e^{-x}, \bar{x}=0$ mas a sequência (A.2) não converge se $x_{0}>1$. Para $f(x)=|x|^{\alpha}$ com $0<\alpha<\frac{1}{2}, \bar{x}=0$ mas a sequência diverge para 


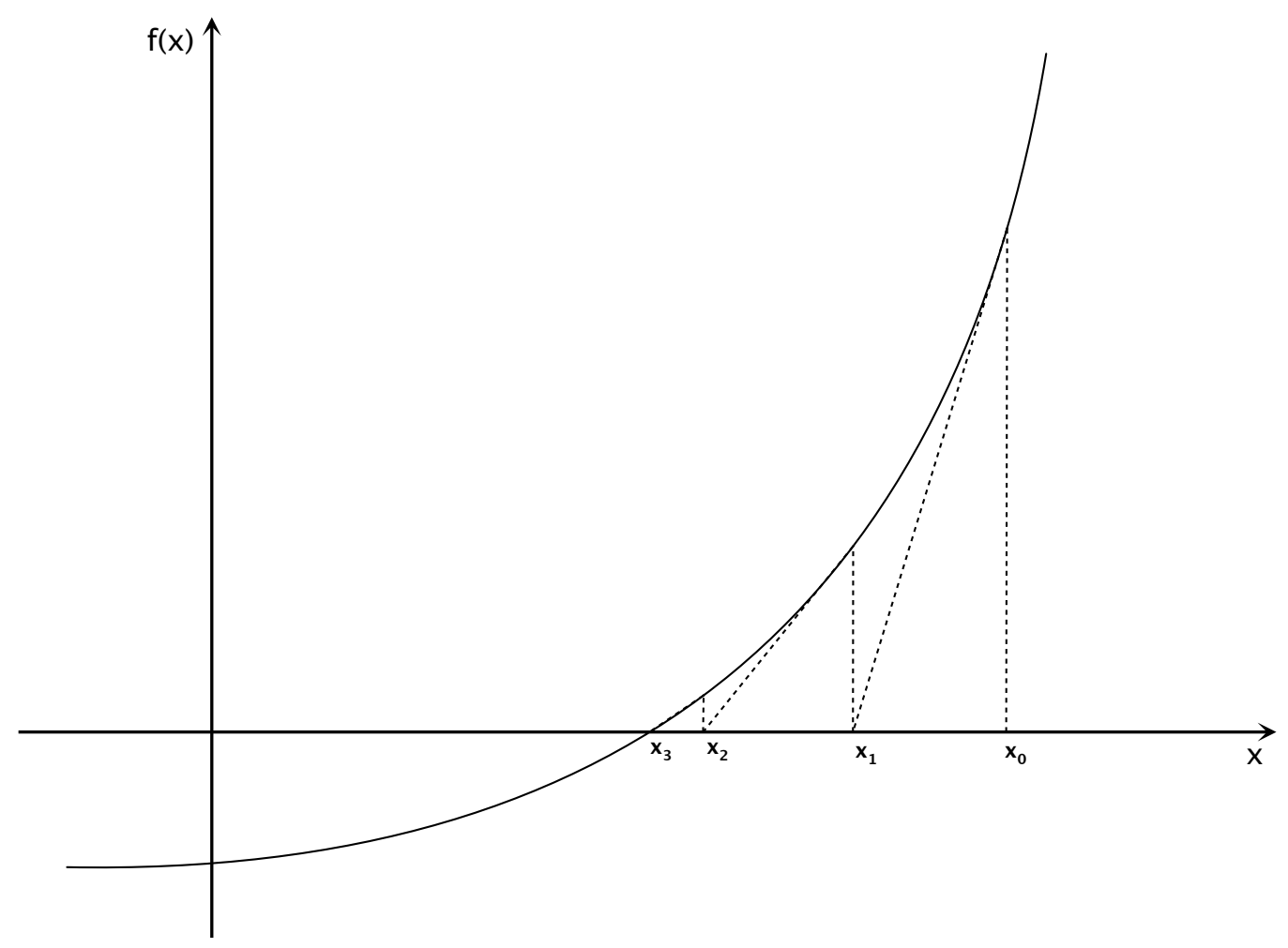

Figura A.1: Passos do método de Newton.

qualquer $x_{0} \neq 0$. Para saber exatamente qual é a vizinhança de convergência, é necessário conhecer o comportamento da função em torno da raiz, especialmente a primeira e a segunda derivada. Se esta vizinhança for pequena, pode-se usar o método da dicotomia para alcançar uma tentativa inicial melhor.

Algoritmo para uma certa tentativa inicial $x_{0}$ :

1. Calcular $f\left(x_{0}\right)$ e $f^{\prime}\left(x_{0}\right)$;

2. Testar se $f\left(x_{0}\right)=0$. Se atender a essa condição, $\bar{x}=x_{0}$ e encerro o algoritmo. Caso contrário, continuo;

3. Calcular $x_{1}=x_{0}-\frac{f\left(x_{0}\right)}{f^{\prime}\left(x_{0}\right)}$;

4. Testar se $\left|x_{1}-x_{0}\right|=0$. Se atender a essa condição, $\bar{x}=x_{1}$ e encerro o algoritmo. Caso contrário, continuo;

5. Definir $x_{0}=x_{1}$ e voltar para o passo 1 . 


\section{A.1.3 Método de busca por gradientes}

Eventualmente as funções das quais queremos obter as raízes serão complexas. Para visualizar as dificuldades de obter raízes de funções complexas, vamos tratálas como vetores em um espaço de duas dimensões. Seja $\vec{F}(\vec{x})=(f(x, y), g(x, y))$, $\vec{x}=(x, y)$ e $\overrightarrow{\vec{x}}$ tal que $f(\bar{x}, \bar{y})=g(\bar{x}, \bar{y})=0$. Esse problema é muito difícil de ser resolvido numericamente, pois na prática deve-se mapear os pontos em que $f(x, y)=0$, que é uma curva em $\mathbb{R}^{2}$, mapear a curva $g(x, y)=0$ e encontrar as interseções das duas curvas.

Para resolver esse problema, podemos definir $h(x, y)=f^{2}(x, y)+g^{2}(x, y)$, onde obviamente $h(x, y) \geq 0$ e $h(x, y)=0$ corresponde a $f(x, y)=g(x, y)=0$. É mais simples encontrar os mínimos locais de $h(x, y)$ e verificar se esse mínimo é próximo o suficiente de $h(x, y)=0$ que encontrar os zeros simultâneos de $f(x, y)$ e $g(x, y)$. Para isso, podemos usar o método de busca por gradientes, onde partindo de uma tentativa inicial $\overrightarrow{x_{0}}=\left(x_{0}, y_{0}\right)$, calculo o gradiente de $h(x, y)$ no ponto $\left(x_{0}, y_{0}\right)$ e tomo como nova tentativa um ponto pertencendo a reta definida pelo gradiente de $h(x, y)$, ou seja, $\overrightarrow{x_{1}}=\overrightarrow{x_{0}}+\lambda \vec{\nabla} h\left(\overrightarrow{x_{0}}\right)$. $\lambda$ é escolhido como o valor que minimiza a função unidimensional $h(\lambda)=h\left(\vec{x}_{1}(\lambda)\right)=h\left(\overrightarrow{x_{0}}+\lambda \vec{\nabla} h\left(\overrightarrow{x_{0}}\right)\right)$. A repetição dessas operações tende a um mínimo local.

Não há garantias de que o mínimo encontrado por este método seja de fato um mínimo global. A taxa de convergência deste método pode ser lenta, especialmente próximo da solução, mas este problema pode ser contornado corrigindo o passo usando o método do gradiente conjugado [48].

$\mathrm{O}$ algoritmo a ser seguido para uma tentativa inicial $\overrightarrow{x_{0}}$ é:

1. Calcular $\vec{\nabla} h(\vec{x})$ no ponto $\vec{x}=\overrightarrow{x_{0}}$;

2. Testar de $\left\|\vec{\nabla} h\left(\overrightarrow{x_{0}}\right)\right\|=0$. Se for o caso, encerro o algoritmo;

3. Calcular $\lambda$ que minimiza a função $h(\lambda)=h\left(\overrightarrow{x_{0}}+\lambda \vec{\nabla} h\left(\overrightarrow{x_{0}}\right)\right)$;

4. Calcular $\overrightarrow{x_{1}}=\overrightarrow{x_{0}}+\lambda \vec{\nabla} h\left(\overrightarrow{x_{0}}\right)$;

5. Testar se $\left\|\overrightarrow{x_{1}}-\overrightarrow{x_{0}}\right\|=0$. Se for o caso, encerro o algoritmo;

6. Voltar pro passo $1 \operatorname{com} x_{0}=x_{1}$.

\section{A.1.4 Raízes de polinômios}

Como mencionado, encontrar um zero de funções complexas é, em geral, uma operação muito complicada, exceto para algumas classes específicas de funções 
complexas. Uma dessas classes são os polinômios, mas antes de descrever métodos de busca de raízes de polinômios é conveniente descrever um esquema para o cálculo do valor de um polinômio em um certo ponto, assim como as suas derivadas, de maneira conveniente.

Seja $p(x)$ um polinômio de grau $N$ expresso por

$$
p(x)=\sum_{k=0}^{N} A_{k} x^{k} .
$$

Se $N=5$, por exemplo, podemos calcular o valor de $p(x)$ em um ponto $x_{0}$ de uma maneira ingênua como

$$
\begin{aligned}
p\left(x_{0}\right)= & A_{0}+A_{1} \cdot x_{0}+A_{2} \cdot x_{0} \cdot x_{0}+A_{3} \cdot x_{0} \cdot x_{0} \cdot x_{0} \\
& +A_{4} \cdot x_{0} \cdot x_{0} \cdot x_{0} \cdot x_{0}+A_{5} \cdot x_{0} \cdot x_{0} \cdot x_{0} \cdot x_{0} \cdot x_{0},
\end{aligned}
$$

onde a operação de multiplicação foi denotada explicitamente para mostrar a quantidade de somas ou multiplicações envolvida no cálculo de $p\left(x_{0}\right)$. Tal operação envolve $\frac{(N+1)(N+2)}{2}-1$ operações de soma ou multiplicação. A operação

$$
p\left(x_{0}\right)=A_{0}+x_{0} \cdot\left(A_{1}+x_{0} \cdot\left(A_{2}+x_{0} \cdot\left(A_{3}+x_{0} \cdot\left(A_{4}+x_{0} \cdot A_{5}\right)\right)\right)\right)
$$

envolve apenas $2 N$ operações.

Sejam as sequências $B_{k}$ e $C_{k}$ definidas como $B_{N-1}=A_{N}, B_{k}=A_{k+1}+x_{0} \cdot B_{k+1}$ para $k=N-2, \ldots, 0,-1$ e $C_{N-2}=B_{N-1}, C_{k}=B_{k+1}+x_{0} \cdot C_{k+1}$ para $k=N-$ $3, \ldots, 0,-1$. Podemos escrever o polinômio (A.4) como

$$
p(x)=\left(x-x_{0}\right)\left(B_{N-1} x^{N-1}+B_{N-2} x^{N-2}+\cdots+B_{1} x+B_{0}\right)+B_{-1}
$$

ou

$p(x)=\left(x-x_{0}\right)^{2}\left(C_{N-2} x^{N-2}+C_{N-3} x^{N-3}+\cdots+C_{1} x+C_{0}\right)+C_{-1}\left(x-x_{0}\right)+B_{-1}$.

Após um número de operações proporcional a $N$, temos $p\left(x_{0}\right)=B_{-1}, p^{\prime}\left(x_{0}\right)=$ $C_{-1}{ }^{1}$ e se $p\left(x_{0}\right)=0$, a sequência $b_{k}$ já nos dá o polinômio que resulta da divisão de $p(x)$ por $\left(x-x_{0}\right)$ e repetimos o método numérico para este novo polinômio de grau menor até acharmos todas as raízes do polinômio (A.4).

\section{A.1.5 Método de Laguerre}

Supomos que todas as raízes $\alpha_{k}, k=1, \ldots, N$ de um polinômio $p(x)$ sejam reais e , portanto, que possam ser ordenadas, $\alpha_{1}<\alpha_{2}<\cdots<\alpha_{N}$. Definindo $\alpha_{0}=-\infty$ e $\alpha_{N+1}=+\infty$ podemos definir os intervalos $I_{k}=\left(\alpha_{k}, \alpha_{k+1}\right)$.

\footnotetext{
${ }^{1}$ Com uma sequência adicional, podemos obter $p^{\prime \prime}\left(x_{0}\right)$.
} 
Seja $x_{0}$ uma tentativa inicial para uma raiz de $p(x) . x_{0} \in I_{k}$ para algum $k=$ $0,1, \ldots, N$. É possível construir uma parábola que é negativa em $x_{0}$ e positiva em cada uma das raízes de $p(x)$. As duas raízes dessa parábola devem pertencer ao mesmo intervalo $I_{k}$ ao qual $x_{0}$ pertence. Uma dessas raízes será escolhida como nova tentativa $x_{1}$ de raiz de $p(x)$.

A parábola

$$
\phi(x)=\left(x_{0}-x\right)^{2} S(\lambda)-(\lambda-x)^{2}
$$

com

$$
S(\lambda)=\sum_{i=1}^{N}\left(\frac{\lambda-\alpha_{i}}{x_{0}-\alpha_{i}}\right)^{2}
$$

é negativa em $x_{0}$ e positiva em qualquer $\alpha_{k}$ se $p\left(x_{0}\right) \neq 0$. No entanto, precisamos dos valores das raízes de $p(x)$ para calcular $S(\lambda)$. Esse problema pode ser contornado fazendo $\lambda=\mu+x_{0}$,

$$
S(\lambda)=\mu^{2} \sum_{i=1}^{N} \frac{1}{\left(x_{0}-\alpha_{i}\right)^{2}}+2 \mu \sum_{i=1}^{N} \frac{1}{x_{0}-\alpha_{i}}+N .
$$

Escrevendo

$$
p(x)=\left(x-\alpha_{1}\right)\left(x-\alpha_{2}\right) \cdots\left(x-\alpha_{N}\right)
$$

podemos escrever as somatórias da equação (A.11) como

$$
\sum_{i=1}^{N} \frac{1}{x_{0}-\alpha_{i}}=\frac{p^{\prime}\left(x_{0}\right)}{p\left(x_{0}\right)}
$$

$\mathrm{e}$

$$
\sum_{i=1}^{N} \frac{1}{\left(x_{0}-\alpha_{i}\right)^{2}}=\frac{\left[p^{\prime}\left(x_{0}\right)\right]^{2}-p\left(x_{0}\right) p^{\prime \prime}\left(x_{0}\right)}{p^{2}\left(x_{0}\right)} .
$$

O parâmetro $\lambda$ ou $\mu$ é escolhido de modo que as raízes da parábola tenham a maior distância possível. Como essas raízes pertencem a um mesmo intervalo, esse último critério faz com que a próxima tentativa seja o mais próximo possível das raízes de $p(x)$, o que leva a uma convergência rápida.

Calculando as raízes da parábola e substituindo pelos valores de $p\left(x_{0}\right)$ e suas derivadas, obtemos

$$
x_{1}=x_{0}-\frac{N p\left(x_{0}\right)}{p^{\prime}\left(x_{0}\right) \pm \sqrt{H\left(x_{0}\right)}},
$$

onde

$$
H\left(x_{0}\right)=(N-1)\left\{(N-1)\left[p^{\prime}\left(x_{0}\right)\right]^{2}-N p^{\prime \prime}\left(x_{0}\right) p\left(x_{0}\right)\right\}
$$


O sinal no denominador de (A.15) deve ser o mesmo de $p^{\prime}\left(x_{0}\right)$ para a próxima tentativa ter o menor deslocamento a partir de $x_{0}$. Por construção, as raízes da parábola construída por este método estão próximas às raízes de $p(x)$ que definem o intervalo ao qual $x_{0}$ pertence. Então a expressão (A.15) calcula um valor $x_{1}$ próximo a alguma raiz de $p(x)$, que será escolhido como próxima tentativa de raiz de $p(x)$.

Este método possui uma convergência cúbica, mais rápido que o método de Newton, mas precisa do cálculo de $p\left(x_{0}\right), p^{\prime}\left(x_{0}\right)$ e $p^{\prime \prime}\left(x_{0}\right)$. Como este método trata especificamente de polinômios, o cálculo extra é feito sem grandes custos. O método converge para qualquer escolha de $x_{0}$. Se alguma raiz não for simples, a convergência é apenas linear. Se alguma raiz for complexa, nada garante que o método converge para essa raiz, mas é raro o método não convergir mesmo para raízes complexas.

Partindo de uma tentativa inicial $x_{0}$, repetimos:

1. Calcular $p\left(x_{0}\right), p^{\prime}\left(x_{0}\right), p^{\prime \prime}\left(x_{0}\right)$ e $H\left(x_{0}\right)$;

2. Se $p\left(x_{0}\right)=0, x_{0}$ é uma das raízes, encerro o algoritmo;

3. Calcular $x_{1}$ pela expressão (A.15);

4. Se $\left|x_{1}-x_{0}\right|=0$, $x_{0}$ é uma das raízes, encerro o algoritmo;

5. Definir $x_{0}=x_{1}$ e voltar para o passo 1 .

\section{A.1.6 Método de Jenkins-Traub}

Em [49], Jenkins e Traub definem um método de busca de raízes de polinômio sem fazer nenhuma suposição quanto aos elementos do polinômio e às raízes, como por exemplo apenas soluções reais ou multiplicidade simples. A ideia deste método é gerar uma sequência de polinômios que aproxima a sequência $z_{k+1}=$ $z_{k}-f\left(z_{k}\right)$ por $z_{k+1}=z_{k}-\left(z_{k}-\alpha_{j}\right)$ para um $k$ suficientemente grand, onde $\alpha_{j}$ é alguma raiz do polinômio

$$
p(z)=\sum_{i=1}^{N} A_{i} z^{i}=\prod_{j=1}^{p}\left(z-\alpha_{j}\right)^{m_{j}}
$$

$\operatorname{com} A_{N}=1 \mathrm{e}$

$$
\sum_{j=1}^{p} m_{j}=N
$$


ou seja, $p(z)$ possui $p$ raízes, cada uma com multiplicidade $m_{j}$. Definimos a sequência $H^{(k)}(z)$ começando por

$$
H^{(0)}=p^{\prime}(z)=\sum_{j=1}^{p} m_{j} P_{j}(z),
$$

onde

$$
P_{j}(z)=\frac{p(z)}{z-\alpha_{j}}
$$

tendo, em cada iteração, a forma

$$
H^{(k)}(z)=\sum_{j=1}^{p} c_{j}^{(k)} P_{j}(z),
$$

que é um polinômio de grau $N-1$ com termo de ordem máxima igual a $\sum_{j=1}^{p} c_{j}^{(k)}$.

Definindo $\tilde{H}^{(k)}(z)$ como

$$
\tilde{H}^{(k)}(z)=\frac{H^{(k)}(z)}{\sum_{j=1}^{p} c_{j}^{(k)}},
$$

a sequência

$$
z_{k+1}=z_{k}-\frac{p\left(z_{k}\right)}{\tilde{H}^{(k+1)}\left(z_{k}\right)}
$$

tende a $\alpha_{1}$ se a sequência $H^{(k)}(z)$ tender a $c_{1}^{(k)} P_{1}(z)$, ou seja, a razão $\frac{c_{j}^{(k)}}{c_{1}^{(k)}}$ deve tender a 0 para $j \neq 1$ após um $k$ suficientemente grande. Com esta construção, a equação (A.23) é uma sequência da forma $z_{k+1}=z_{k}-f\left(z_{k}\right)$ que tende a $z_{k+1}=\alpha_{j}$.

Construindo $Q^{(k)}(z)$ da forma

$$
Q^{(k)}(z)=H^{(k)}(z)-\frac{H^{(k)}\left(z_{k}\right)}{p\left(z_{k}\right)} p(z)
$$

temos um polinômio de grau $N$ cujo termo de ordem mais alta é $-\frac{H^{(k)}\left(z_{k}\right)}{p\left(z_{k}\right)}$. Não há riscos de divisão por zero pois se por algum acaso $p\left(z_{k}\right)=0$, encontramos uma raiz e podemos encerrar o algoritmo. Por construção, $Q^{(k)}\left(z_{k}\right)=0$, então a divisão deste polinômio por $z-z_{k}$ é exata. Definimos então

$$
H^{(k+1)}=\frac{Q^{(k)}(z)}{z-z_{k}}
$$


Após uma manipulação algébrica, vista com detalhes em [50], pode-se mostrar que

$$
H^{(k+1)}(z)=\sum_{j=1}^{p} c_{j}^{(k+1)} P_{j}(z)=\sum_{j=1}^{p} \frac{c_{j}^{(k)}}{\alpha_{j}-z_{k}} P_{j}(z),
$$

de onde podemos concluir que

$$
c_{j}^{(k+1)}=\frac{c_{j}^{(k)}}{\alpha_{j}-z_{k}}=\frac{m_{j}}{\prod_{l=0}^{k}\left(\alpha_{j}-z_{l}\right)} .
$$

Na equação anterior, $z_{l}$ pode ser uma sequência qualquer. Os coeficientes $c_{j}^{(k)}$ são sempre diferentes de zero para todo $j$ e $k$.

É desejável que o algoritmo ache primeiro a raiz de menor módulo. Ordenando as raízes por módulo, $\left|\alpha_{1}\right|<\left|\alpha_{2}\right|<\cdots<\left|\alpha_{N}\right|$, se escolhermos a sequência $z_{l}=0$ temos

$$
\frac{c_{j}^{(k)}}{c_{1}^{(k)}}=\frac{m_{j}}{m_{1}}\left(\frac{\alpha_{1}}{\alpha_{j}}\right)^{k} \text {. }
$$

Para um $k$ suficientemente grande, a equação (A.23) tende a $t_{k+1}=\alpha_{1}$ com uma taxa de convergência proporcional a $\left|\frac{\alpha_{1}}{\alpha_{2}}\right|$, que pode ser muito próxima de 1, especialmente se $\alpha_{1}$ e $\alpha_{2}$ forem complexos conjugados. Calculamos então a sequência $H^{(k)}(z)$ com a sequência $z_{k}=0$ apenas até um número fixo $M$ de iterações.

Após $M$ iterações, queremos acentuar as diferenças entre possíveis raízes de módulo próximo a $\left|\alpha_{1}\right|$. Seja $\tilde{z}$ definido como

$$
\tilde{z}=\beta e^{i \theta}
$$

onde $\beta$ é a única raiz real positiva de

$$
x^{N}+\left|A_{N-1}\right| x^{N-1}+\cdots+\left|A_{1}\right| x-\left|A_{0}\right|=0
$$

e $\theta$ é um número real aleatório. Por um teorema demonstrado por Cauchy, que pode ser visto em [50], $\beta$ é um limite inferior de $\left|\alpha_{j}\right|$.

Depois de um certo número $L-M$ de iterações a razão

$$
\frac{c_{j}^{(L)}}{c_{1}^{(L)}}=\frac{m_{j}}{m_{1}}\left(\frac{\alpha_{1}}{\alpha_{j}}\right)^{M}\left(\frac{\alpha_{1}-\tilde{z}}{\alpha_{j}-\tilde{z}}\right)^{L-M}
$$

favorece a raiz mais próxima a $\tilde{z}$ e a aproximação (A.23) favorece esta raiz específica. Este estágio é repetido até que as condições

$$
\left|z_{k+1}-z_{k}\right| \leq \frac{1}{2}\left|z_{k}\right|
$$


$\mathrm{e}$

$$
\left|z_{k}-z_{k-1}\right| \leq \frac{1}{2}\left|z_{k-1}\right|,
$$

ainda com $z_{k+1}$ dado por (A.23), sejam satisfeitas.

No terceiro e último estágio, as tentativas de raiz de $p(z)$ são atualizadas a partir de uma sequência de números complexos. Por um teorema demonstrado em [50], mostra-se que se as condições (A.32) e (A.33) forem satisfeitas, a sequência

$$
z_{k+1}=z_{k}-\frac{p\left(z_{k}\right)}{\tilde{H}\left(z_{k}\right)}
$$

converge para alguma raiz de $p(z)$. Essa convergência é quadrática como no Método de Newton, e mais lenta que no Método de Laguerre. No entanto, este método funciona para quaisquer valores complexos de $A_{j}$ e $\alpha_{j}$ e não há perda de convergência se alguma raiz tiver multiplicidade maior que 1.

Para o algoritmo, sabendo os coeficientes $A_{k}$ de $p(z)$ e $H^{(0)}(z)$, no primeiro estágio, temos $z_{k}=0$ e começando por $k=0$ repetimos os seguintes passos:

1. Calcular $p\left(z_{k}\right)$ e $H^{(k)}\left(z_{k}\right)$;

2. Se $p\left(z_{k}\right)=0$ encerrar o algoritmo;

3. Calcular $Q(z)$ usando a expressão (A.24);

4. Calcular $H^{(k+1)}$ dividindo $Q(z)$ por $z-z_{k}$;

5. Dividir $H^{(k+1)}$ pelo termo de maior grau para obter $\tilde{H}^{(k+1)}$;

6. Enquanto $k<M$, voltar para o passo $1 \operatorname{com} k=k+1$.

No segundo estágio, $z=\tilde{z}$ dado por (A.29), definimos $t_{-1}=t_{0}=t_{1}=0 \mathrm{e}$ repetimos:

1. Calcular $p\left(z_{k}\right)$ e $H^{(k)}\left(z_{k}\right)$;

2. Se $p\left(z_{k}\right)=0$, encerrar o algoritmo;

3. Calcular $Q(z)$ usando a expressão A.24;

4. Calcular $H^{(k+1)}$ dividindo $Q(z)$ por $z-z_{k}$;

5. Dividir $H^{(k+1)}$ pelo termo de maior grau para obter $\tilde{H}^{(k+1)}$;

6. Calculo $t_{1}$ como o valor de $t_{k+1}$ da expressão (A.23); 
7. Se $\left|t_{1}-t_{0}\right|<\frac{1}{2}\left|t_{0}\right|$ e $\left|t_{0}-t_{-1}\right|<\frac{1}{2}\left|t_{-1}\right|$, ir para o terceiro estágio. Caso contrário, fazer $t_{-1}=t_{0}, t_{0}=t_{1}, k=k+1 \mathrm{e}$ voltar para o passo 1 deste estágio.

No terceiro estágio, começamos com $z_{0}$ dado pelo valor de $z_{k+1}$ dado por (A.34) e repetimos:

1. Calcular $p\left(z_{0}\right)$ e $H^{(k)}\left(z_{0}\right)$;

2. Se $p\left(z_{0}\right)=0$, encerrar o algoritmo;

3. Calcular $Q(z)$ usando a expressão (A.24);

4. Calcular $H^{(k+1)}$ dividindo $Q(z)$ por $z-z_{0}$;

5. Dividir $H^{(k+1)}$ pelo termo de maior grau para obter $\tilde{H}^{(k+1)}$;

6. Calcular $z_{1}$ pela expressão (A.34);

7. Se $\left|z_{1}-z_{0}\right|=0$, encerrar o algoritmo. Caso contrário, fazer $z_{0}=z_{1}, k=k+1$ e volto para o passo 1 deste estágio.

\section{A.1.7 Polinômios mal condicionados}

Wilkinson define, em [51], o que é um polinômio mal condicionado. Seja o polinômio

$$
p(x)=\sum_{k=0}^{N} A_{k} x^{k}
$$

Definimos $a_{k}=A_{k}+\delta_{k}$, onde $\delta_{k}$ é um erro inerente ao cálculo feito para obter $A_{k}$ e $x_{0}=\overline{x_{0}}+\varepsilon$, onde $\overline{x_{0}}$ é a raiz verdadeira de $p(x), \varepsilon$ é o erro numérico desta raiz e $x_{0}$ é a raiz do polinômio com coeficientes $a_{k}$. Partindo destas definições, obtemos

$$
p\left(x_{0}\right)=0=\sum_{k=0}^{N}\left(A_{k}+\delta_{K}\right)\left(\overline{x_{0}}+\varepsilon\right)^{k}=\sum_{k=0}^{N} A_{k}{\overline{x_{0}}}^{k}+\varepsilon p^{\prime}\left(\overline{x_{0}}\right)+\sum_{k=0}^{N} \delta_{k}{\overline{x_{0}}}^{k}+\cdots
$$

onde omitimos termos de segunda ordem em $\delta_{k}$ e $\varepsilon$. Podemos então, em primeira ordem, estimar o tamanho de $\varepsilon$ em função de $\delta_{k}$ da forma

$$
|\varepsilon| \simeq \frac{\left|\sum_{k=0}^{N} \delta_{k} \overline{x_{0}} K\right|}{\left|p^{\prime}\left(\overline{x_{0}}\right)\right|}
$$


Se $p^{\prime}\left(\overline{x_{0}}\right)$ não for muito pequeno e $\delta_{k}$ forem pequenos, $x_{0}$ é uma boa aproximação para $\overline{x_{0}}$, porém em alguns casos patológicos o erro pode ser da ordem de $\overline{x_{0}}$. Por exemplo, o polinômio

$$
p(x)=\prod_{k=1}^{20}(x+k)
$$

possui uma raiz $\overline{x_{0}}=-20 \mathrm{com}\left|p^{\prime}\left(\overline{x_{0}}\right)\right|=19$ !. Seja $\delta_{k}=0$ para qualquer $k \neq 19 \mathrm{e}$ $\delta_{19}=2^{-23}$. Qualquer método numérico encontrará essa raiz com um erro

$$
|\varepsilon| \simeq \frac{2^{-23}(20)^{19}}{19 !} \simeq 4,4
$$

Mais detalhes deste problema técnico podem ser vistos em [50].

\section{A.2 Integração de funções}

\section{A.2.1 Método do ponto intermediário}

Seja $m$ um número real entre $a$ e $b$. Aproximamos a integral de $f(x)$ entre $a$ e $b$ como a área do quadrado de base $(b-a)$ e altura $f(m)$.

$$
\int_{a}^{b} f(x) \mathrm{d} x \simeq(b-a) f(m)
$$

Existe um $m \in[a, b]$ para o qual a aproximação acima é exata, mas não temos como saber quem é esse $m$ sem resolver o problema. Normalmente a melhor escolha é $m=\frac{a+b}{2}$.

Dividindo o intervalo $[a, b]$ em $N$ intervalos de tamanho $\Delta x=\frac{b-a}{N}$, rotulando os pontos por

$$
\begin{aligned}
x_{0} & =a, \\
x_{i} & =a+i \Delta x, \\
x_{N} & =a+N \Delta x=b,
\end{aligned}
$$

aproximamos a integral por uma expressão mais precisa dada por

$$
\int_{a}^{b} f(x) \mathrm{d} x \simeq \sum_{i=0}^{N-1} f\left(x_{i}+\frac{\Delta x}{2}\right) \Delta x .
$$

O erro numérico $\varepsilon$ da aproximação (A.44) é dado por

$$
\varepsilon=\frac{(b-a)}{24} \Delta x^{2} \max _{\xi \in[a, b]}\left|f^{\prime \prime}(\xi)\right| .
$$




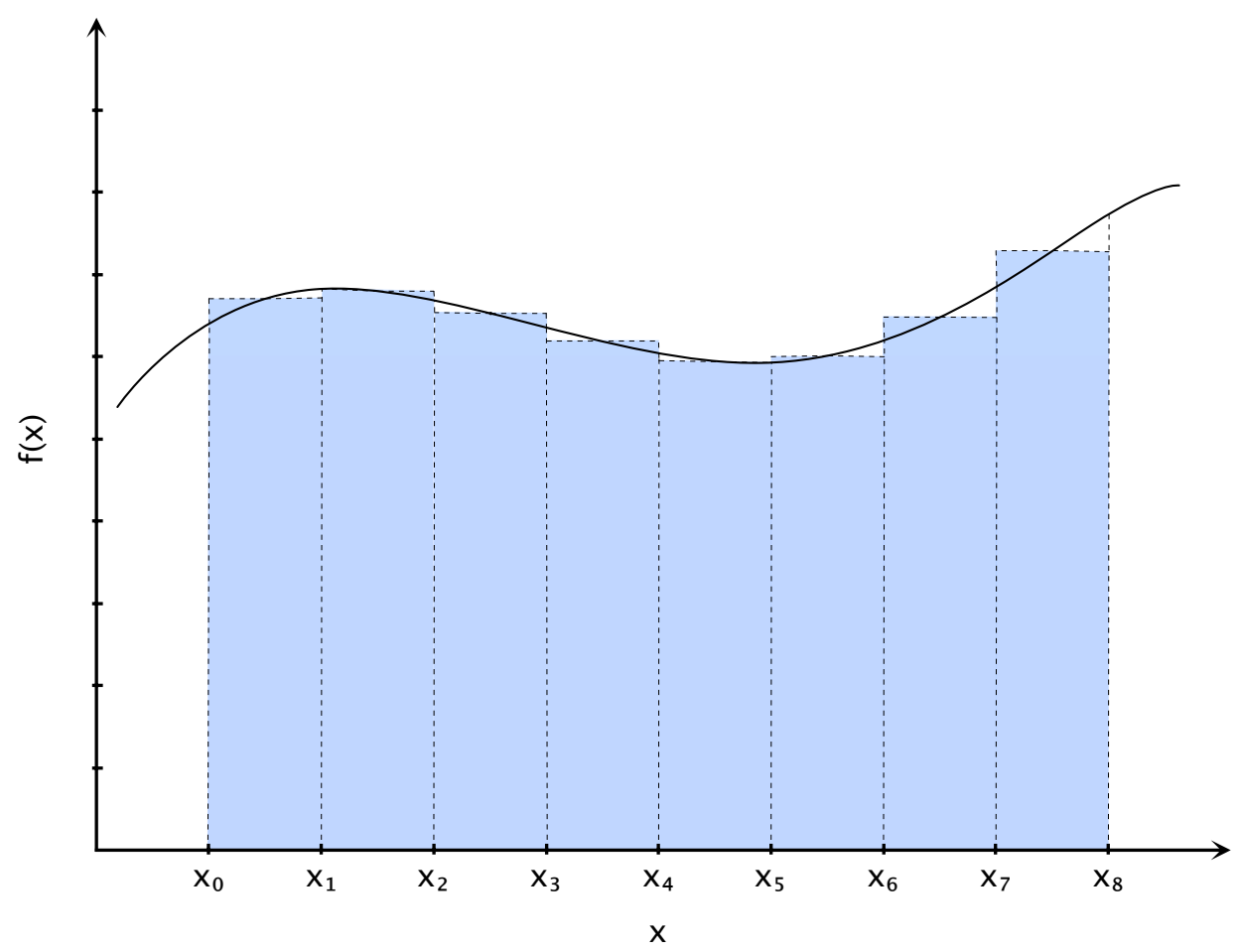

Figura A.2: Aproximação dada pelo método do ponto intermediário.

\section{A.2.2 Método do Trapézio}

Este método é semelhante ao anterior, mas ao invés de usarmos o valor da função em um ponto qualquer do intervalo $[a, b]$, usamos os extremos do intervalo e aproximamos a integral como a área do trapézio abaixo da reta que liga o ponto $(a, f(a))$ com o ponto $(b, f(b))$.

$$
\int_{a}^{b} f(x) \mathrm{d} x \simeq \frac{b-a}{2}(f(a)+f(b)) .
$$

Assim como no método anterior, dividimos o intervalo em $N$ intervalos definidos pelos pontos

$$
\begin{aligned}
x_{0} & =a, \\
x_{i} & =a+i \Delta x, \\
x_{N} & =a+N \Delta x=b,
\end{aligned}
$$

e aproximamos a integral de $f(x)$ por

$$
\int_{a}^{b} f(x) \mathrm{d} x \simeq \frac{\Delta x}{2}\left(f\left(x_{0}\right)+2 f\left(x_{1}\right)+2 f\left(x_{2}\right)+\cdots+2 f\left(x_{N-1}\right)+f\left(x_{N}\right)\right) .
$$




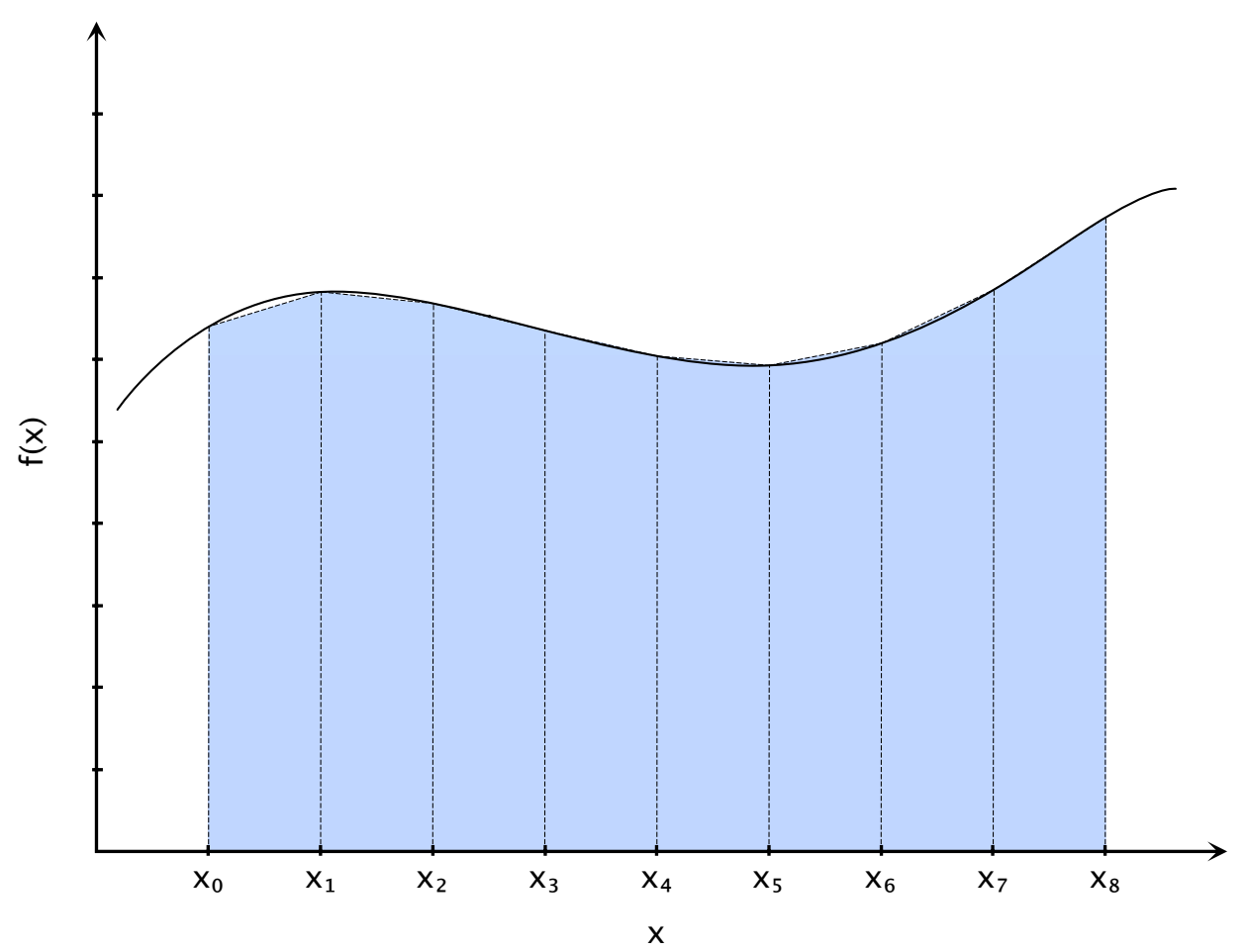

Figura A.3: Aproximação dada pelo método do trapézio.

O erro desta aproximação é dado por

$$
\varepsilon=\frac{b-a}{12} \Delta x^{2} \max _{\xi \in[a, b]}\left|f^{\prime \prime}(\xi)\right|
$$

\section{A.2.3 Regra de Simpson}

O método do trapézio pode ser entendido formalmente como a integral do polinômio interpolador de $f(x)$ de primeiro grau que passa pelos pontos $(a, f(a))$ e $(b, f(b))$, que é o polinômio de grau mais alto que pode ser definido com apenas dois pontos. Escolhendo um ponto $m \in(a, b)$, podemos calcular o polinômio interpolador de Lagrange que passa pelos pontos $(a, f(a)),(m, f(m))$ e $(b, f(b))$, dado por

$$
P(x)=f(a) \frac{(x-m)(x-b)}{(a-m)(a-b)}+f(m) \frac{(x-a)(x-b)}{(m-a)(m-b)}+f(b) \frac{(x-a)(x-m)}{(b-a)(b-m)},
$$

e aproximar a integral de $f(x)$ pela integral do polinômio $P(x)$. 
Com a escolha $m=\frac{a+b}{2}$ é fácil verificar que

$$
\int_{a}^{b} P(x) \mathrm{d} x=\frac{b-a}{6}\left[f(a)+4 f\left(\frac{a+b}{2}\right)+f(b)\right] .
$$

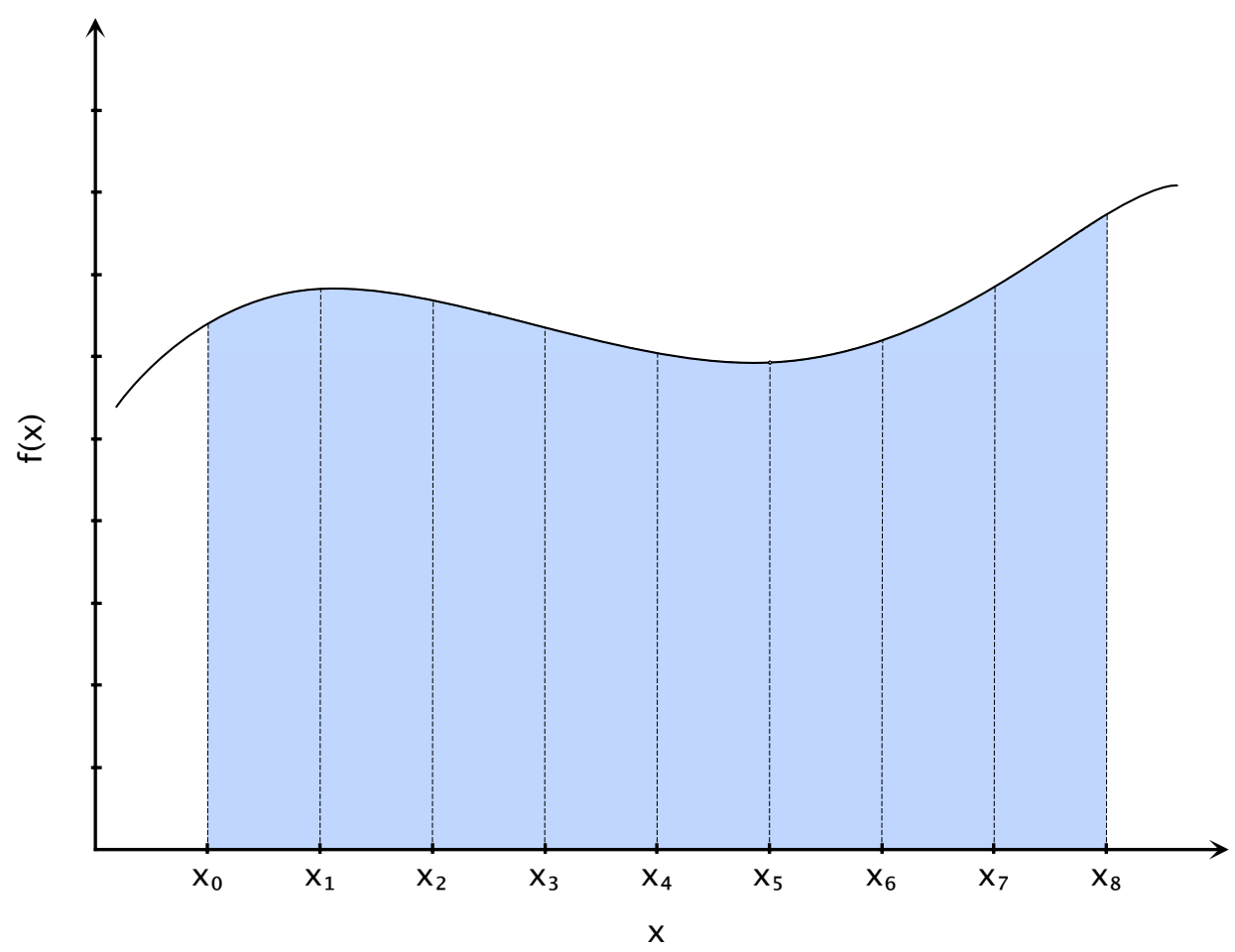

Figura A.4: Aproximação dada pela regra de Simpson.

Novamente dividimos o intervalo $[a, b]$ em $N$ intervalos definidos pelos pontos

$$
\begin{aligned}
x_{0} & =a, \\
x_{i} & =a+i \Delta x, \\
x_{N} & =a+N \Delta x=b,
\end{aligned}
$$

com a diferença de que $N$ deve ser um número par. Os pontos rotulados por um número par são os extremos dos subintervalos e os pontos rotulados por números ímpares são o ponto médio do subintervalo.

A integral é então aproximada por

$$
\begin{gathered}
\int_{a}^{b} f(x) \mathrm{d} x \simeq \frac{\Delta x}{3} \quad\left[f\left(x_{0}\right)+4 f\left(x_{1}\right)+2 f\left(x_{2}\right)+4 f\left(x_{3}\right)+\cdots\right. \\
\left.+2 f\left(x_{N-2}\right)+4 f\left(x_{N-1}\right)+f\left(x_{N}\right)\right]
\end{gathered}
$$


com um erro dado por

$$
\frac{b-a}{180} \Delta x^{4} \max _{\xi \in[a, b]}\left|f^{(4)}(\xi)\right|
$$

Pode-se, com mais pontos entre o intervalo $(a, b)$, interpolar polinômios de ordens mais altas, mas o número de operações a serem feitas a mais para conseguir uma aproximação melhor que a regra de Simpson é maior que simplesmente diminuir o passo deste método.

\section{A.3 Equações Diferenciais Ordinárias}

\section{A.3.1 Método de Euler}

Seja o caso mais simples de uma equação diferencial ordinária,

$$
\frac{\mathrm{d} x}{\mathrm{~d} t}=f(x, t)
$$

Partindo da expansão de Taylor

$$
x(t+h)=x(t)+\frac{\mathrm{d} x}{\mathrm{~d} t} h+\frac{1}{2} \frac{\mathrm{d}^{2} x}{\mathrm{~d} t^{2}} h^{2}+\cdots
$$

podemos calcular o valor de $x(t+h)$ a partir de (A.59) por

$$
x(t+h)=x(t)+h f(x, t)+O\left(h^{2}\right) .
$$

Partindo de uma condição inicial $x\left(t_{0}\right)=x_{0}$, calculamos o valor de $x(t)$ para $t=t_{0}+k h$, sendo $k$ um número inteiro.

\section{A.3.2 Método de Runge-Kutta}

Este método é uma generalização do método de Euler usando aproximações de ordens mais altas para $x(t+h)$, desenvolvido por Runge e Kutta em [52; 53]. Partindo novamente da forma mais simples de uma equação diferencial ordinária

$$
\frac{\mathrm{d} x}{\mathrm{~d} t}=f(x, t)
$$

queremos partir de uma condição inicial $x\left(t_{0}\right)=x_{0}$ calcular o valor de $x(t)$ em $t=t_{0}+k h$ para $k$ inteiros. 
Na expansão de Taylor para $x(t+h)$, substituimos a derivada $n$-ésima derivada por

$$
\begin{aligned}
\left(\frac{\mathrm{d}}{\mathrm{d} t}\right)^{n} x(t) & =\left(\frac{\mathrm{d}}{\mathrm{d} t}\right)^{n-1} f(x, t)=\left(\frac{\partial}{\partial t}+\frac{\mathrm{d} x}{\mathrm{~d} t} \frac{\partial}{\partial x}\right)^{n-1} \\
& =\left(\frac{\partial}{\partial t}+f(x, t) \frac{\partial}{\partial x}\right)^{n-1} f(x, t)
\end{aligned}
$$

usando a equação diferencial (A.62).

Denotando

$$
D=\frac{\partial}{\partial t}+f(x, t) \frac{\partial}{\partial x} .
$$

podemos, de forma mais simples, escrever

$$
\begin{aligned}
& \left(\frac{\partial}{\partial t}+f(x, t) \frac{\partial}{\partial x}\right)^{2}=D^{2} f+\frac{\partial f}{\partial x} D \\
& \left(\frac{\partial}{\partial t}+f(x, t) \frac{\partial}{\partial x}\right)^{3}=D^{3} f+\frac{\partial f}{\partial x} D^{2} f+\left(\frac{\partial f}{\partial x}\right)^{2} D f+3 D f D \frac{\partial f}{\partial x}
\end{aligned}
$$

e aproximar a expansão de $x(t+h)$ até quarta ordem como

$$
\begin{aligned}
x(t+h) & =x(t)+\left\{h f+\frac{h^{2}}{2} D f+\frac{h^{3}}{3 !}\left(D^{2} f+\frac{\partial f}{\partial x} D f\right)\right. \\
& \left.+\frac{h^{4}}{4 !}\left[D^{3} f+\frac{\partial f}{\partial x} D^{2} f+\left(\frac{\partial f}{\partial x}\right)^{2} D f+3 D f D \frac{\partial f}{\partial x}\right]\right\}+O\left(h^{5}\right) .
\end{aligned}
$$

Queremos escrever a aproximação (A.67) como uma soma de $p$ termos $k_{i}$ com pesos $w_{i}$ expressa por

$$
x(t+h)=x(t)+\sum_{i=1}^{p} w_{i} k_{i},
$$

onde $w_{i}$, por ser um peso, não depende de $t$ ou $x$, e $k_{i}$ é o valor de $f(x, t)$ calculando em um ponto com $t$ com um deslocamento inicialmente arbitrário e $x$ somado a uma combinação linear dos $k_{j}$ anteriores, ou seja,

$$
k_{i}=h f\left(t+\alpha_{i} h, x+\sum_{j=1}^{i-1} \beta_{i j} k_{j}\right)
$$

Para aproximar a expansão de Taylor de quarta ordem são necessários quatro termos $k_{i}$. É um trabalho extremamente tedioso expandir os termos $k_{i}$ até quarta 
ordem. Ao comparar com a expansão de Taylor, para obter os termos da equação (A.67) vezes alguma constante que não depende de $f(x, t)$, é preciso impor

$$
\alpha_{i}=\sum_{j=1}^{i-1} \beta_{i j}
$$

para $i=2,3,4$. Ao compararmos os oito termos, um de primeira ordem, um de segunda ordem, dois de terceira ordem e quatro de quarta ordem em $h$, obtemos o sistema

$$
\begin{aligned}
& w_{1}+w_{2}+w_{3}+w_{4}=1, \\
& w_{2} \alpha_{2}+w_{3} \alpha_{3}+w_{4} \alpha_{4}=\frac{1}{2}, \\
& w_{2} \alpha_{2}^{2}+w_{3} \alpha_{3}^{2}+w_{4} \alpha_{4}^{2}=\frac{1}{3}, \\
& w_{3} \alpha_{2} \beta_{32}+w_{4}\left(\alpha_{2} \beta_{42}+\alpha_{3} \beta_{43}\right)=\frac{1}{6}, \\
& w_{2} \alpha_{2}^{3}+w_{3} \alpha_{3}^{3}+w_{4} \alpha_{4}^{3}=\frac{1}{4}, \\
& w_{3} \alpha_{2}^{2} \beta_{32}+w_{4}\left(\alpha_{2}^{2} \beta_{42}+\alpha_{3}^{2} \beta_{43}\right)=\frac{1}{12}, \\
& w_{3} \alpha_{2} \alpha_{3} \beta_{32}+w_{4}\left(\alpha_{2} \beta_{42}+\alpha_{3} \beta_{43}\right) \alpha_{4}=\frac{1}{8}, \\
& w_{4} \beta_{32} \beta_{43} \alpha_{2}=\frac{1}{24} .
\end{aligned}
$$

O sistema acima e as imposições (A.70) formam um conjunto de onze equações para treze variáveis. Restam então dois graus de liberdade. Cada escolha possível de $\left(\alpha_{2}, \alpha_{3}\right)$, por exemplo, gera um método de Runge-Kutta diferente.

Se nos limitarmos apenas até termos de primeira ordem na equação (A.68), a única solução é $w_{1}=1$, que resulta em

$$
x(t+h)=x(t)+h f(x, t)+O\left(h^{2}\right),
$$

que é o método de Euler, ou seja, podemos entender o método de Runge-Kutta como uma generalização do método de Euler para aproximações de ordem mais alta. Se $f(x, t)$ for uma função apenas de $t$, este método de Runge-Kutta torna-se o método do ponto intermediário com escolha de $m=a$.

Para uma aproximação de segunda ordem, precisamos de dois termos na equação (A.68), com os quais temos o sistema

$$
\begin{aligned}
w_{1}+w_{2} & =1, \\
w_{2} \alpha_{2} & =\frac{1}{2},
\end{aligned}
$$


e a imposição $\alpha_{2}=\beta_{21}$, que vem da imposição (A.70). A escolha de $\alpha_{2}$ determina o método de segunda ordem. Escolhendo $\alpha_{2}=\frac{1}{2}$, temos a aproximação

$$
x(t+h)=x(t)+h f\left(x+\frac{h}{2} f(x, t), t+\frac{h}{2}\right)+O\left(h^{3}\right) .
$$

Se $f(x, t)$ depende apenas de $t$, este método torna-se o método do ponto intermediário com escolha de $m$ igual ao ponto médio do intervalo entre $t$ e $t+h$.

Escolhendo $\alpha_{2}=1$, temos a aproximação

$$
x(t+h)=x(t)+\frac{h}{2}\{f(x, t)+f[x+h f(x, t), t+h]\}+O\left(h^{3}\right),
$$

que é a mesma expressão do método do trapézio se $f(x, t)$ depende apenas de $t$.

Finalmente para quarta ordem, escolhendo $\alpha_{2}=\alpha_{3}=\frac{1}{2}$, temos

$$
x(t+h)=x(t)+\frac{1}{6}\left(k_{1}+2 k_{2}+2 k_{3}+k_{4}\right)+O\left(h^{5}\right)
$$

com

$$
\begin{aligned}
& k_{1}=h f(x, t), \\
& k_{2}=h f\left(x+\frac{k_{1}}{2}, t+\frac{h}{2}\right), \\
& k_{3}=h f\left(x+\frac{k_{2}}{2}, t+\frac{h}{2}\right), \\
& k_{4}=h f\left(x+k_{3}, t+h\right) .
\end{aligned}
$$

Se $f(x, t)$ for uma função apenas de $t$, este método torna-se o a regra de Simpson.

Para uma aproximação de ordem $n>4$, é necessário uma soma com $p>n$ na equação (A.68), em particular, para $n=5$, o valor de $p$ mínimo é $p=6$, para $n=6, p \geq 7$. Para $m \geq 7, p \geq m+2$. Por esse motivo, para uma aproximação de ordem maior que quatro, o número de contas a serem feitas em cada passo é tão grande que em geral é melhor simplesmente diminuir o tamanho do passo no método de quarta ordem. Neste trabalho, em todas as aplicações do método de Runge-Kutta a ordem da aproximação é $m=4$. Mais detalhes das contas para deduzir o método e estimativa do erro em [50].

\section{A.4 Equações Diferenciais Parciais}

A forma mais geral de uma equação diferencial parcial linear de segunda ordem, em duas dimensões é

$$
a \frac{\partial^{2} u}{\partial x^{2}}+b \frac{\partial^{2} u}{\partial x \partial y}+c \frac{\partial^{2} u}{\partial y^{2}}+d \frac{\partial u}{\partial x}+e \frac{\partial u}{\partial y}+f u+g=0 .
$$


Com uma transformação de coordenadas $\tilde{x}=\tilde{x}(x, y)$ e $\tilde{y}=\tilde{y}(x, y)$, a equação (A.89) assume a forma

$$
A \frac{\partial^{2} u}{\partial \tilde{x}^{2}}+B \frac{\partial^{2} u}{\partial \tilde{x} \partial \tilde{y}}+C \frac{\partial^{2} u}{\partial \tilde{y}^{2}}+\cdots=0
$$

onde omitimos termos de até primeira ordem. As novas funções $A, B$ e $C$ se relacionam com as antigas da forma

$$
B^{2}-4 A C=\left(b^{2}-4 a c\right)\left(\frac{\partial \tilde{x}}{\partial x} \frac{\partial \tilde{y}}{\partial y}-\frac{\partial \tilde{x}}{\partial y} \frac{\partial \tilde{y}}{\partial x}\right)^{2}
$$

O termo elevado ao quadrado é o Jacobiano da transformação. O sinal de $b^{2}-$ $4 a c$ é mantido e podemos classificar as equações diferenciais parciais de segunda ordem pelo sinal dessa quantidade.

A equação (A.89) é hiperbólica se $b^{2}-4 a c>0$ e por uma transformação conveniente, pode ser escrita da forma

$$
\frac{\partial^{2} u}{\partial \tilde{x}^{2}}-\frac{\partial^{2} u}{\partial \tilde{y}^{2}}+\cdots=0
$$

Se $b^{2}-4 a c=0$, a equação diferencial é parabólica e pode ser escrita na forma

$$
\frac{\partial^{2} u}{\partial \tilde{x}^{2}}+\cdots=0
$$

e finalmente, se $b^{2}-4 a c<0$, a equação diferencial é elíptica e pode ser escrita na forma

$$
\frac{\partial^{2} u}{\partial \tilde{x}^{2}}+\frac{\partial^{2} u}{\partial \tilde{y}^{2}}+\cdots=0
$$

Novamente os termos de até primeira ordem foram omitidos. A transformação que leva a equação (A.89) para uma dessas formas conveniente é obtida resolvendo um sistema de equações diferenciais parciais de primeira ordem.

\section{A.4.1 Método de Diferenças Finitas}

No ponto $(x, y)$, expandimos $u(x, y)$ em uma série de Taylor,

$$
u(x+\Delta x, y+\Delta y)=\sum_{n=0}^{\infty} \sum_{k=0}^{n} \frac{1}{n !}\left(\begin{array}{l}
n \\
k
\end{array}\right) \frac{\partial^{n} u}{\partial x^{k} \partial y^{n-k}}(x, y) \Delta x^{k} \Delta y^{n-k},
$$


da qual estamos interessados apenas em termos de até segunda ordem.

$$
\begin{array}{r}
f(x+\Delta x, y+\Delta y)=f(x, y)+\left[\frac{\partial f}{\partial x}(x, y) \Delta x+\frac{\partial f}{\partial y}(x, y) \Delta y\right] \\
+\frac{1}{2}\left[\frac{\partial^{2} f}{\partial x^{2}}(x, y) \Delta x^{2}+2 \frac{\partial^{2} f}{\partial x \partial y}(x, y) \Delta x \Delta y+\frac{\partial^{2} f}{\partial y^{2}}(x, y) \Delta y^{2}\right]+O\left(\Delta^{3}\right) .
\end{array}
$$

Uma derivada parcial de primeira ordem em $x$ pode ser aproximada por

$$
\frac{\partial u}{\partial x}=\frac{u(x+\Delta x, y)-u(x, y)}{\Delta x}+O(\Delta x) .
$$

Essa aproximação tem um erro proporcional a $\Delta x$. A aproximação

$$
\frac{\partial u}{\partial x}=\frac{u(x+\Delta x, y)-u(x-\Delta x, y)}{2 \Delta x}+O\left(\Delta x^{2}\right)
$$

possui um erro proporcional a $\Delta x^{2}$ e portanto é uma aproximação melhor. Analogamente, podemos aproximar todos os termos de (A.89) como diferenças de $u(x, y)$ calculada em pontos diferentes.

$$
\begin{aligned}
\frac{\partial u}{\partial x} & =\frac{u(x+\Delta x, y)-u(x-\Delta x, y)}{2 \Delta x}+O\left(\Delta x^{2}\right), \\
\frac{\partial u}{\partial y} & =\frac{u(x, y+\Delta y)-u(x, y-\Delta y)}{2 \Delta y}+O\left(\Delta y^{2}\right), \\
\frac{\partial^{2} u}{\partial x^{2}} & =\frac{u(x+\Delta x, y)-2 u(x, y)+u(x-\Delta x, y)}{\Delta x^{2}}+O\left(\Delta x^{2}\right), \\
\frac{\partial^{2} u}{\partial x^{2}}= & \frac{u(x, y+\Delta y)-2 u(x, y)+u(x, y-\Delta y)}{\Delta y^{2}}+O\left(\Delta y^{2}\right), \\
\frac{\partial^{2} u}{\partial x \partial y}= & \frac{u(x+\Delta x, y+\Delta y)-u(x+\Delta x, y-\Delta y)-u(x-\Delta x, y+\Delta y)+u(x-\Delta x, y-\Delta y)}{4 \Delta x \Delta y}
\end{aligned}
$$

Tomando apenas pontos pertencentes a um conjunto discreto expresso por

$$
\begin{aligned}
& x=x_{0}+j \Delta x, \\
& y=y_{0}+l \Delta y,
\end{aligned}
$$

podemos escrever a função $u(x, y)$ calculada em pontos diferentes como

$$
\begin{aligned}
& u(x, y)=u\left(x_{0}+j \Delta x, y_{0}+l \Delta y\right)=u_{j, l}, \\
& u(x+\Delta x, y)=u_{j+1, l}, \\
& u(x-\Delta x, y)=u_{j-1, l} \\
& u(x, y+\Delta y)=u_{j, l+1} \\
& u(x, y-\Delta y)=u_{j, l-1} \\
& u(x+\Delta x, y+\Delta y)=u_{j+1, l+1}
\end{aligned}
$$


e as aproximações das derivadas parciais de $u(x, y)$ como

$$
\begin{aligned}
\frac{\partial^{2} u}{\partial x^{2}}(x, y) & =\frac{u_{j+1, l}-2 u_{j, l}+u_{j-1, l}}{\Delta x^{2}}+O\left(\Delta x^{2}\right) \\
\frac{\partial^{2} u}{\partial x \partial y}(x, y) & =\frac{u_{j+1, l+1}-u_{j-1, l+1}-u_{j+1, l-1}+u_{j-1, l-1}}{4 \Delta x \Delta y}+O(\Delta x \Delta y) \\
\frac{\partial^{2} u}{\partial y^{2}}(x, y) & =\frac{u_{j, l+1}-2 u_{j, l}+u_{j, l-1}}{\Delta y^{2}}+O\left(\Delta y^{2}\right) \\
\frac{\partial u}{\partial x}(x, y) & =\frac{u_{j+1, l}-u_{j-1, l}}{2 \Delta x}+O\left(\Delta x^{2}\right) \\
\frac{\partial u}{\partial y}(x, y) & =\frac{u_{j, l+1}-u_{j, l-1}}{2 \Delta y}+O\left(\Delta y^{2}\right)
\end{aligned}
$$
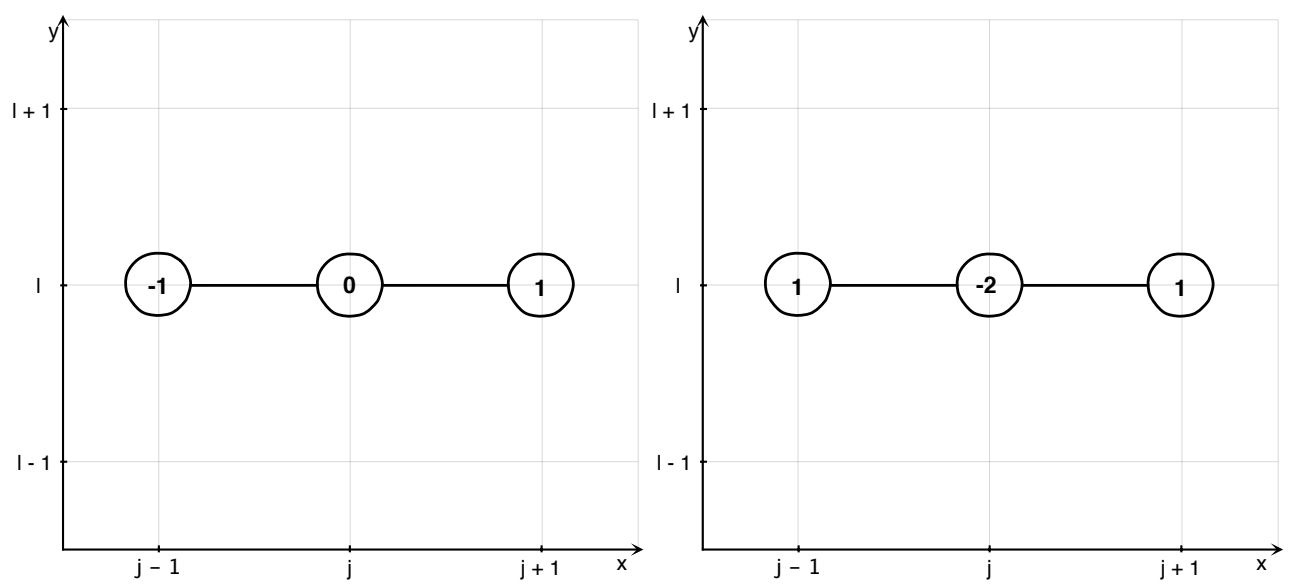

Figura A.5: Células computacionais para $\frac{\partial f}{\partial x}(x, y) \mathrm{e} \frac{\partial^{2} f}{\partial x^{2}}(x, y)$.

\section{A.4.2 Equações hiperbólicas}

Seja o caso mais simples para uma equação diferencial hiperbólica,

$$
-\frac{\partial^{2} u}{\partial t^{2}}+\frac{\partial^{2} u}{\partial x^{2}}=0
$$

com as condições iniciais $u\left(x, t_{0}\right)=f(x) \mathrm{e} \frac{\partial u}{\partial t}\left(x, t_{0}\right)=g(x)$. Com a discretização $x=x_{0}+j \Delta x$ e $t=t_{0}+l \Delta x$ escrevemos a equação (A.118) como

$$
-\frac{u_{j, l+1}-2 u_{j, l}+u_{j, l-1}}{\Delta t^{2}}+\frac{u_{j+1, l}-2 u_{j, l}+u_{j-1, l}}{\Delta x^{2}}=0 .
$$



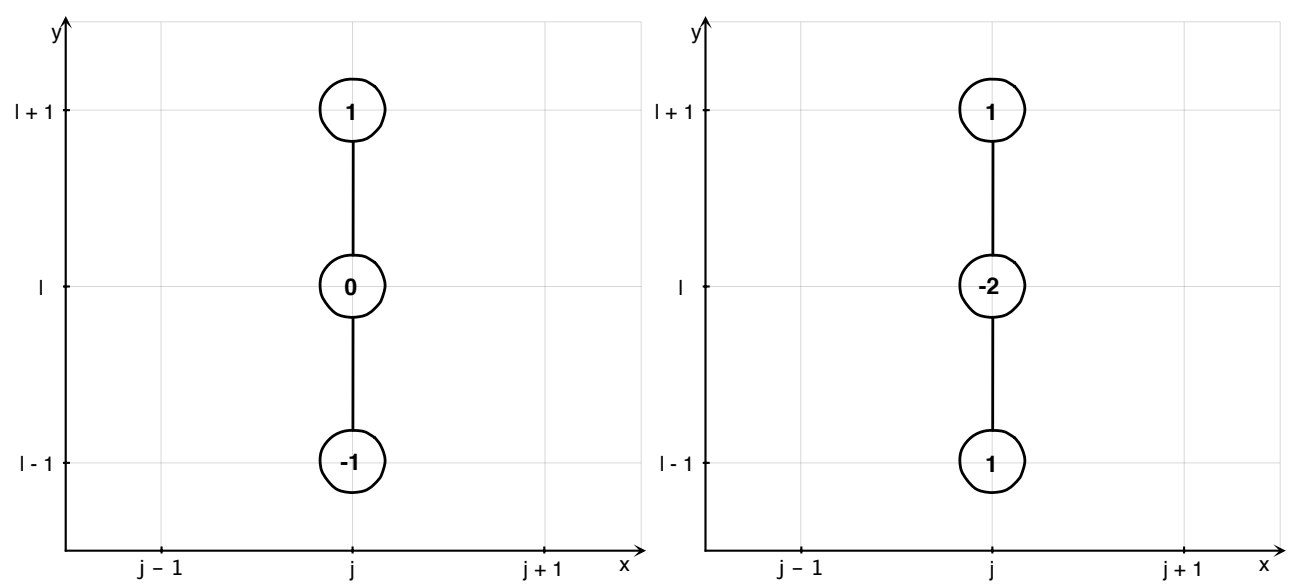

Figura A.6: Células computacionais para $\frac{\partial f}{\partial y}(x, y)$ e $\frac{\partial^{2} f}{\partial y^{2}}(x, y)$.
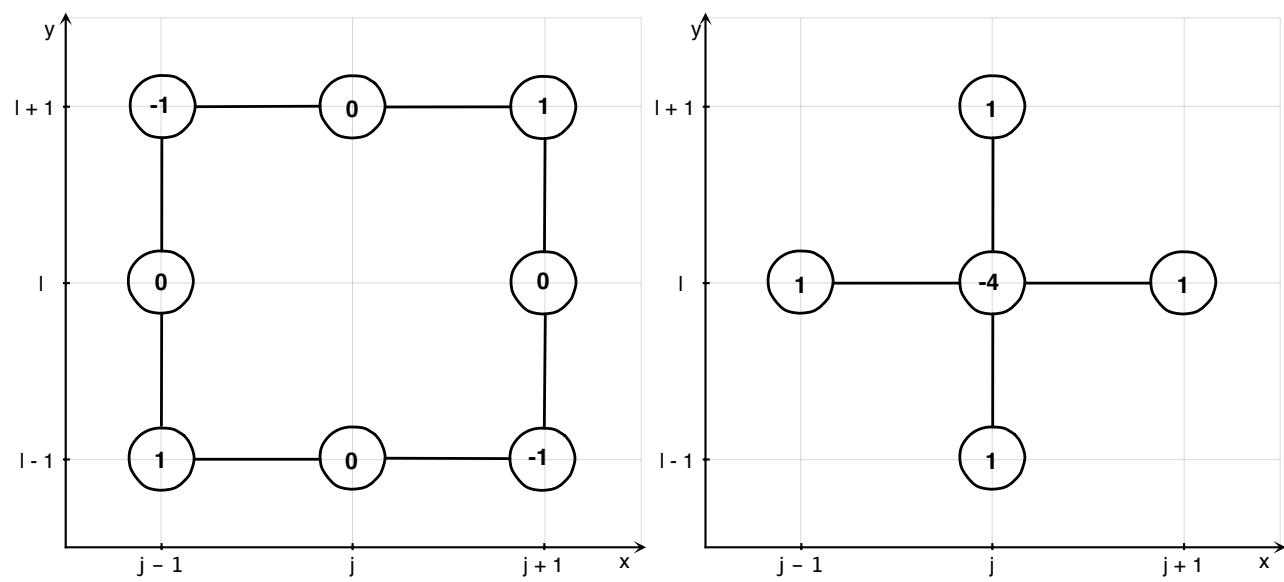

Figura A.7: Células computacionais para $\frac{\partial^{2} f}{\partial x \partial y}(x, y)$ e $\nabla^{2} f(x, y)$.

As condições iniciais definem os valores de $u_{j, l}$ rotulados por $l=0$ e $l=1$,

$$
\begin{aligned}
& u_{j, 0}=f\left(x_{0}+j \Delta x\right)=f_{j}, \\
& u_{j, 1}=f_{j}+g_{j} \Delta t+\frac{1}{2} f_{j}^{\prime \prime} \Delta t^{2} .
\end{aligned}
$$

Definindo as condições iniciais em uma região $\left[x_{0}, x_{M A X}\right] \operatorname{com} x_{M A X}=x_{0}+$ $N \Delta x$, podemos calcular $u_{j, l}$ na região definida por $1<l<\frac{N}{2}$ e $l<j<N-l$ pela 
equação

$$
u_{j, l+1}=-u_{j, l-1}+\frac{\Delta t^{2}}{\Delta x^{2}}\left(u_{j+1, l}+u_{j-1, l}\right)+\left(2-2 \frac{\Delta t^{2}}{\Delta x^{2}}\right) u_{j, l}
$$

A região onde calculamos o valor de $u_{j, l}$ é exibida na figura A.8, onde podemos ver que não há uma dependência de condições de contorno. Como pode ser visto em [22; 23], neste domínio o valor de $u_{j, l}$ depende apenas das condições iniciais se estas condições forem reais e analíticas.

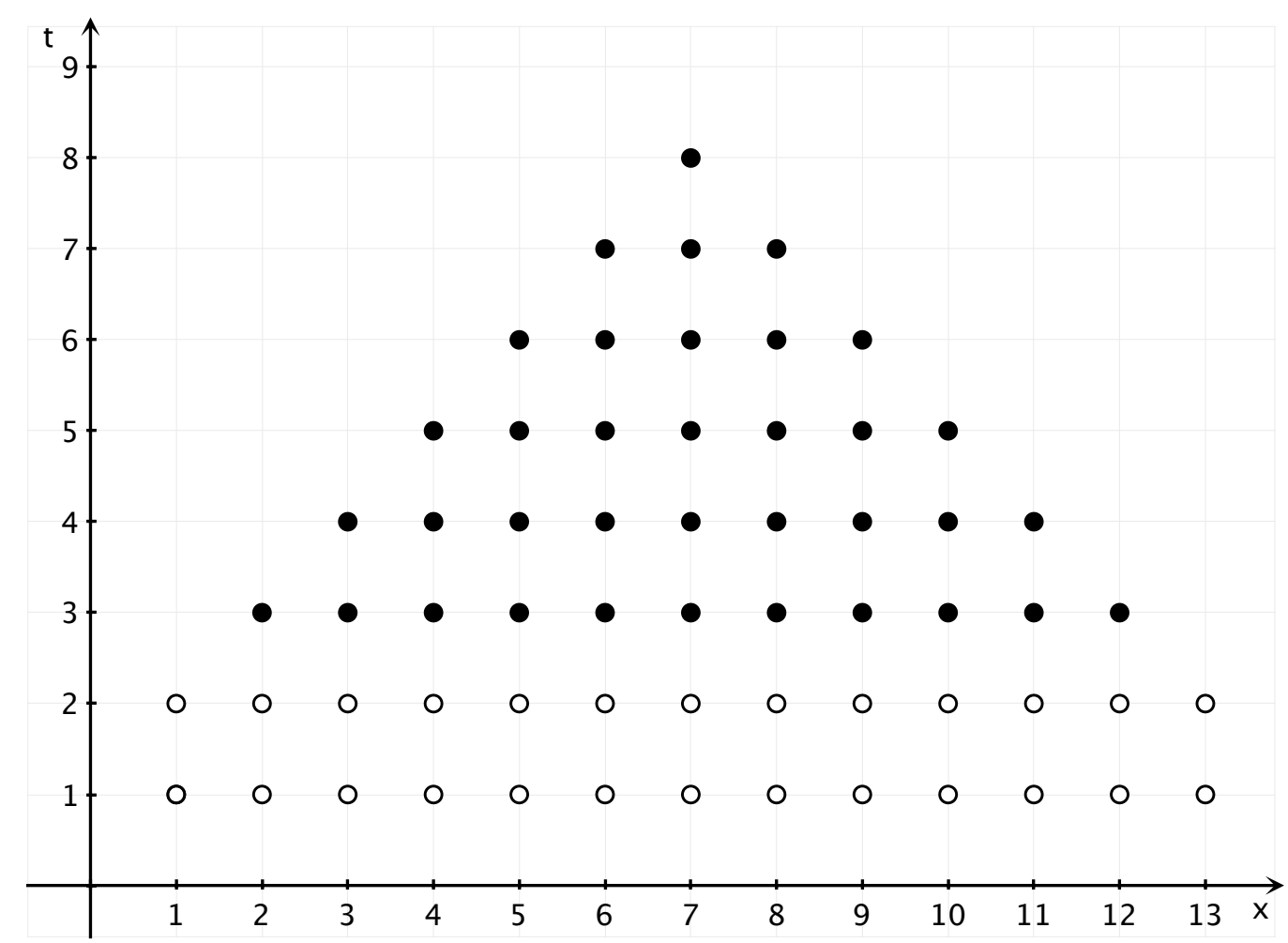

Figura A.8: Domínio de integração de uma equação hiperbólica.

A condição para que a grade numérica esteja dentro do domínio de integração mostrado na figura A.8 é $\Delta t \leq \Delta x$. Se esta condição não for satisfeita, alguns pontos da grade numérica estarão fora do domínio para o qual é garantida a existência e a unicidade. Est condição é conhecida como critério de Courant-FriedrichsLewy para convergência e estabilidade do método de diferenças finitas. 


\section{A.4.3 Estabilidade de von Neumann}

Seja a solução de equação diferencial parcial hiperbólica $u_{j, l}$. Podemos expressar esse valor na forma

$$
u_{j, l}=e^{i j \theta} e^{i l v},
$$

onde $\theta$ é um número real e $v$ complexo. Substituindo a equação acima em (A.119) temos a seguinte relação entre $v$ e $\theta$,

$$
\operatorname{sen}^{2}\left(\frac{v}{2}\right)=\frac{\Delta t^{2}}{\Delta x^{2}} \operatorname{sen}^{2}\left(\frac{\theta}{2}\right) .
$$

Para $v=0, u_{j, 0}$ pode ser entendido como uma transformada de Fourier da condição inicial.

O critério de estabilidade de von Neumann diz que o método de diferenças finitas é estável se $u_{j, l}$ expressa pela equação (A.123) for limitada para todo $l>0$. Ou seja, a solução de $v$ para a equação (A.124) não pode ter parte imaginária negativa para qualquer valor real de $\theta$.

Se $\Delta t<\Delta x$, as soluções da equação (A.124) são todas reais. Se $\Delta t>\Delta x$, para algum valor de $\theta$ real, o lado direito da equação (A.124) é maior que um e as soluções aparecem em pares de complexos conjugados, violando o critério de von Neumann.

\section{A.4.4 Equações parabólicas}

Seja a equação

$$
-\frac{\partial u}{\partial t}+\frac{\partial^{2} u}{\partial x^{2}}=0
$$

com a condição inicial $u\left(x, t_{0}\right)=f(x)$. Como a equação (A.125) é de primeira ordem em $t$ e portanto só tenho uma condição inicial, só posso calcular cada linha rotulada por $l$ a partir da linha anterior, então devo usar como aproximação para a derivada em $t$ a diferença

$$
\frac{\partial u}{\partial t}=\frac{u_{j, l+1}-u_{j, l}}{\Delta t}+O(\Delta t)
$$

A condição inicial define a linha rotulada por $l=0, u_{j, 0}=f_{j}$ e calculamos cada linha a partir da anterior por

$$
u_{j, l+1}=u_{j, l}+\frac{\Delta t}{\Delta x^{2}}\left(u_{j+1, l}-2 u_{j, l}+u_{j-1, l}\right) .
$$

Para testar a estabilidade do método, fazemos $u_{j, l}=e^{i j \theta} e^{i l \lambda}$, substituimos na equação (A.127) e a dividimos por $u_{j, l}$, resultando em

$$
e^{i \lambda}=1-\frac{4 \Delta t}{\Delta x^{2}} \sin ^{2}\left(\frac{\theta}{2}\right) .
$$


A condição $\operatorname{Im} \lambda>0$ implica em $\left|e^{i \lambda}\right| \leq 1$, que para ser válida para qualquer escolha de $\theta$ real deve valer

$$
\frac{4 \Delta t}{\Delta x^{2}} \leq 2
$$

ou seja,

$$
\Delta t \leq \frac{\Delta x^{2}}{2}
$$

\section{A.4.5 Equações elípticas}

Seja a equação

$$
\frac{\partial^{2} u}{\partial x^{2}}+\frac{\partial^{2} u}{\partial y^{2}}=0
$$

que com a discretização $x=x_{0}+j \Delta t$ e $y=y_{0}+l \Delta y$ pode ser escrita como

$$
\frac{u_{j+1, l}-2 u_{j, l}+u_{j-1, l}}{\Delta x^{2}}+\frac{u_{j, l+1}-2 u_{j, l}+u_{j, l-1}}{\Delta y^{2}}=0 .
$$

Neste caso não há problemas com estabilidade de von Neumann, e podemos tomar $\Delta x=\Delta y$ e escrever a equação acima como

$$
u_{j, l}=\frac{1}{4}\left(u_{j+1, l}+u_{j-1, l}+u_{j, l+1}+u_{j, l-1}\right) .
$$

Em outras palavras, o valor de $u_{j, l}$ é a média dos 4 pontos adjascentes na grade, como esperado para uma função harmônica. Definindo uma condição de contorno em uma curva fechada, a equação (A.133) pode ser usada para calcular o valor de $u_{j, l}$ dentro da condição de contorno.

Na figura A.9 mostramos um exemplo de uma grade numérica em que definimos uma condição de contorno (círculos escuros) e não sabemos o valor de $u(x, y)$ nos pontos denotados por uma circunferência. Para cada ponto cujo valor de $u(x, y)$ é desconhecido, escrevemos uma equação atribuindo valores de $j$ e $l$ em A.133.

$$
\begin{aligned}
-4 u_{1,1}+u_{1,0}+u_{0,1}+u_{1,2}+u_{2,1} & =0 \\
-4 u_{2,1}+u_{2,0}+u_{1,1}+u_{2,2}+u_{3,1} & =0 \\
-4 u_{1,2}+u_{1,1}+u_{0,2}+u_{1,3}+u_{2,2} & =0 \\
-4 u_{2,2}+u_{2,1}+u_{1,2}+u_{2,3}+u_{3,2} & =0 .
\end{aligned}
$$

O sistema acima possui quatro equações e quatro variáveis. O problema então está bem definido e possui uma solução única. Para uma grade com $N$ intervalos nas duas dimensões, o número de variáveis e de equações do sistema a ser 


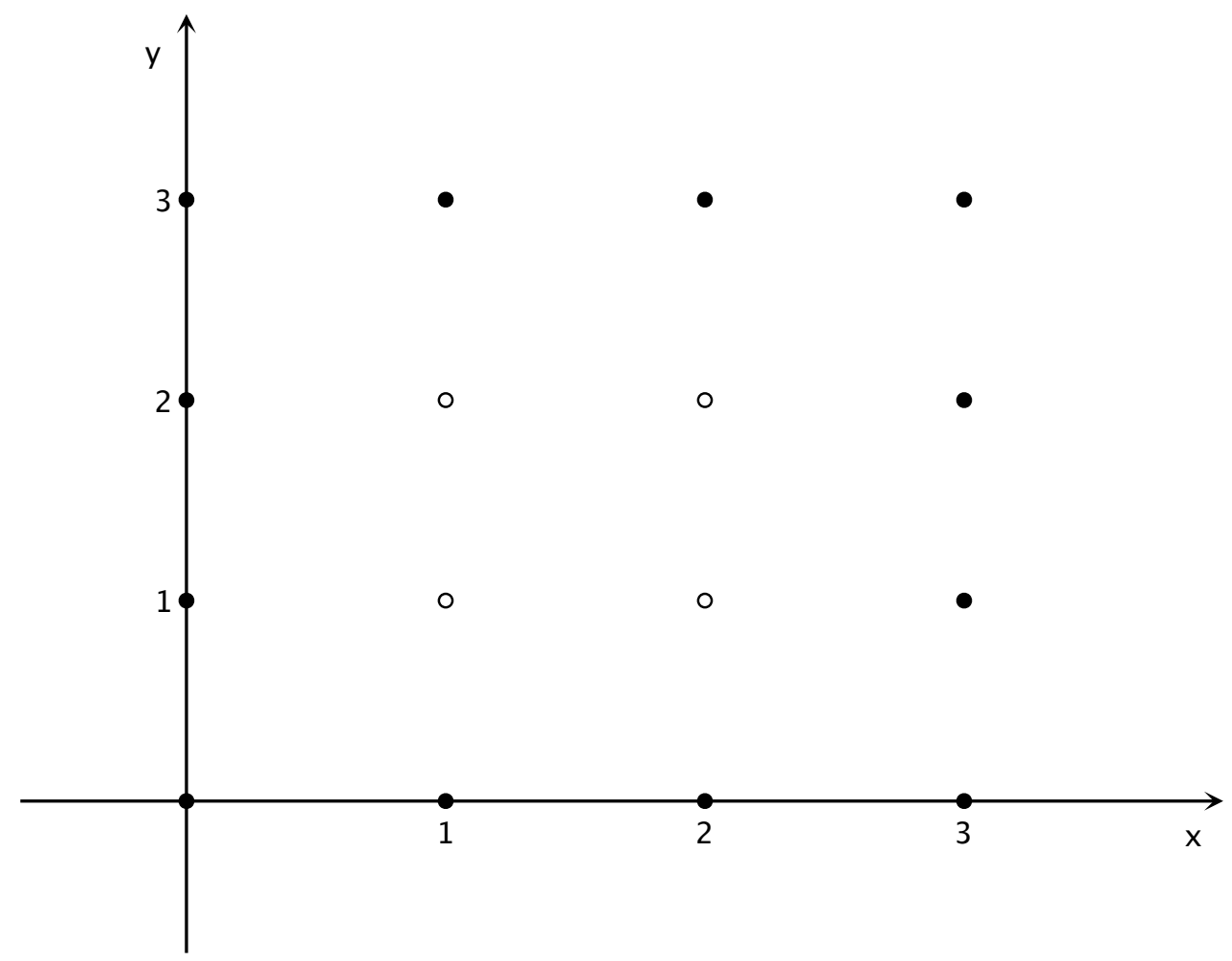

Figura A.9: Domínio de integração de uma equação elíptica.

resolvido é $(N-1)^{2}$ o que leva um tempo longo para ser resolvido. Ao invés de resolver sistemas lineares, podemos definir um conjunto de valores arbitrários para as icógnitas, denotados por $u_{j, l}^{(0)}$ e atualizar estes valores por

$$
u_{j, l}^{(k+1)}=\frac{1}{4}\left(u_{j+1, l}^{(k)}+u_{j-1, l}^{(k)}+u_{j, l+1}^{(k)}+u_{j, l-1}^{(k)}\right) .
$$

Subtraindo $u_{j, l}^{(k)}$ dos dois lados da equação (A.138) temos

$$
u_{j, l}^{(k+1)}-u_{j, l}^{(k)}=\frac{1}{4}\left(u_{j+1, l}^{(k)}-2 u_{j, l}^{(k)}+u_{j-1, l}^{(k)}\right)+\frac{1}{4}\left(u_{j, l+1}^{(k)}-2 u_{j, l}^{(k)}+u_{j, l-1}^{(k)}\right) .
$$

Dividindo por $\Delta t=\frac{\Delta x^{2}}{4}=\frac{\Delta y^{2}}{4}$, temos

$$
\frac{u_{j, l}^{(k+1)}-u_{j, l}^{(k)}}{\Delta t}=\frac{u_{j+1, l}^{(k)}-2 u_{j, l}^{(k)}+u_{j-1, l}^{(k)}}{\Delta x^{2}}+\frac{u_{j, l+1}^{(k)}-2 u_{j, l}^{(k)}+u_{j, l-1}^{(k)}}{\Delta y^{2}} .
$$

A equaç ao (A.140) é equivalente a uma equação de diferenças finitas para a 
equação parabóloca

$$
\frac{\partial u}{\partial t}=\frac{\partial^{2} u}{\partial x^{2}}+\frac{\partial^{2} u}{\partial y^{2}}
$$

que obedece à condição de estabilidade para equações parabólicas. Em outras palavras, um conjunto inicial que obedeça as condições de contorno tende à solução do sistema de equações (A.134) a (A.137).

A convergência das correções pode ser acelerada usando o método de Sobrerrelaxação Sucessiva, definido por

$$
u_{j, l}^{(k+1)}=u_{j, l}^{(k)}+\frac{\omega}{4}\left(u_{j-1, l}^{(k+1)}+u_{j, l+1}^{(k+1)}+u_{j+1, l}^{(k)}+u_{j, l-1}^{(k)}-4 u_{j, l}^{(k)}\right),
$$

$\operatorname{com} \omega=\frac{2}{1-\text { sqrt } 1-e^{2}}$ e $e=1-2 \operatorname{sen}^{2}\left(\frac{\pi}{2 N}\right)$. Neste caso, os valores $u_{j, l}^{(k)}$ devem ser corrigidos na ordem definida na figura A.10.

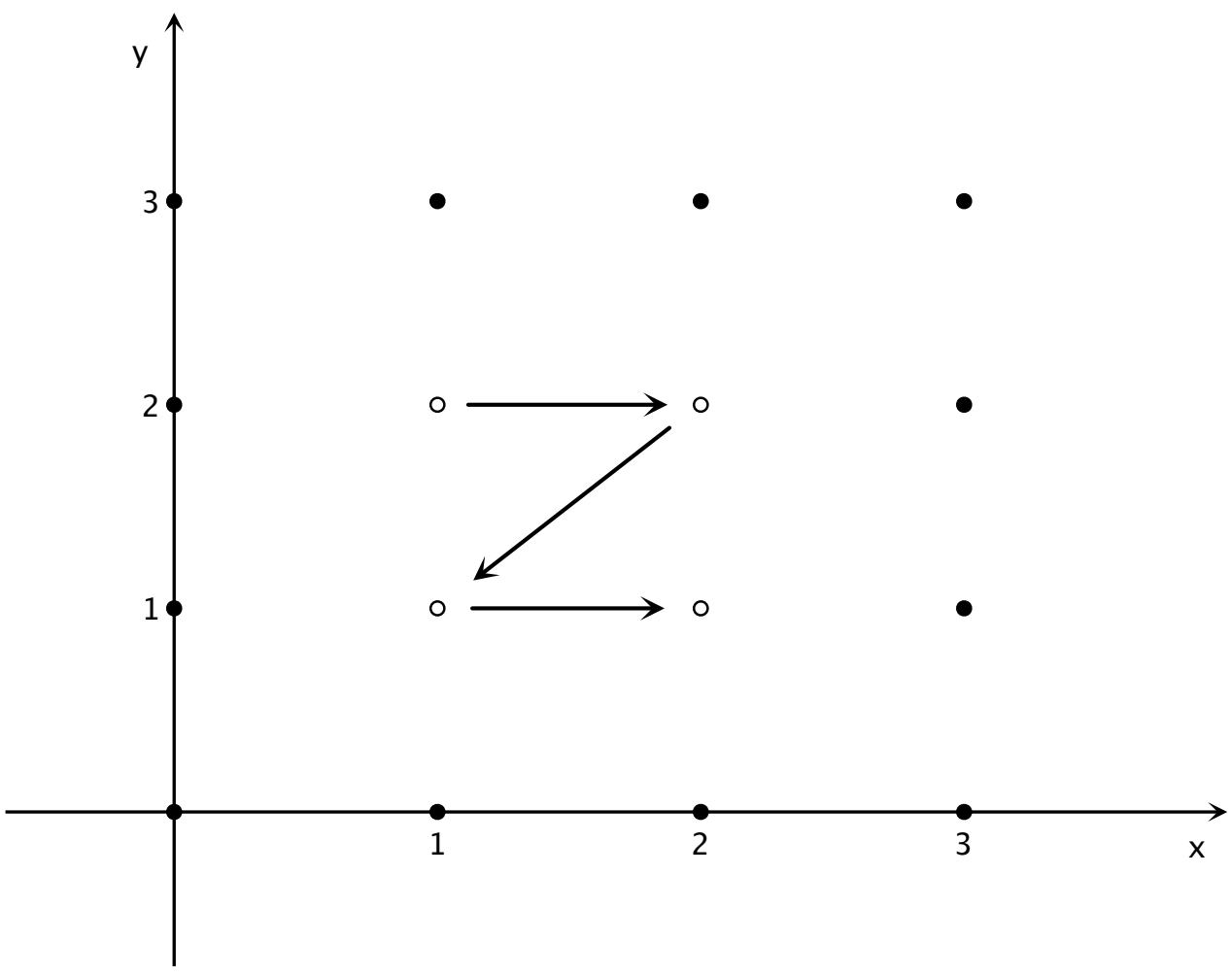

Figura A.10: Ordem de relaxação. 


\section{Apêndice B}

\section{Código numérico do método de diferanças finitas.}

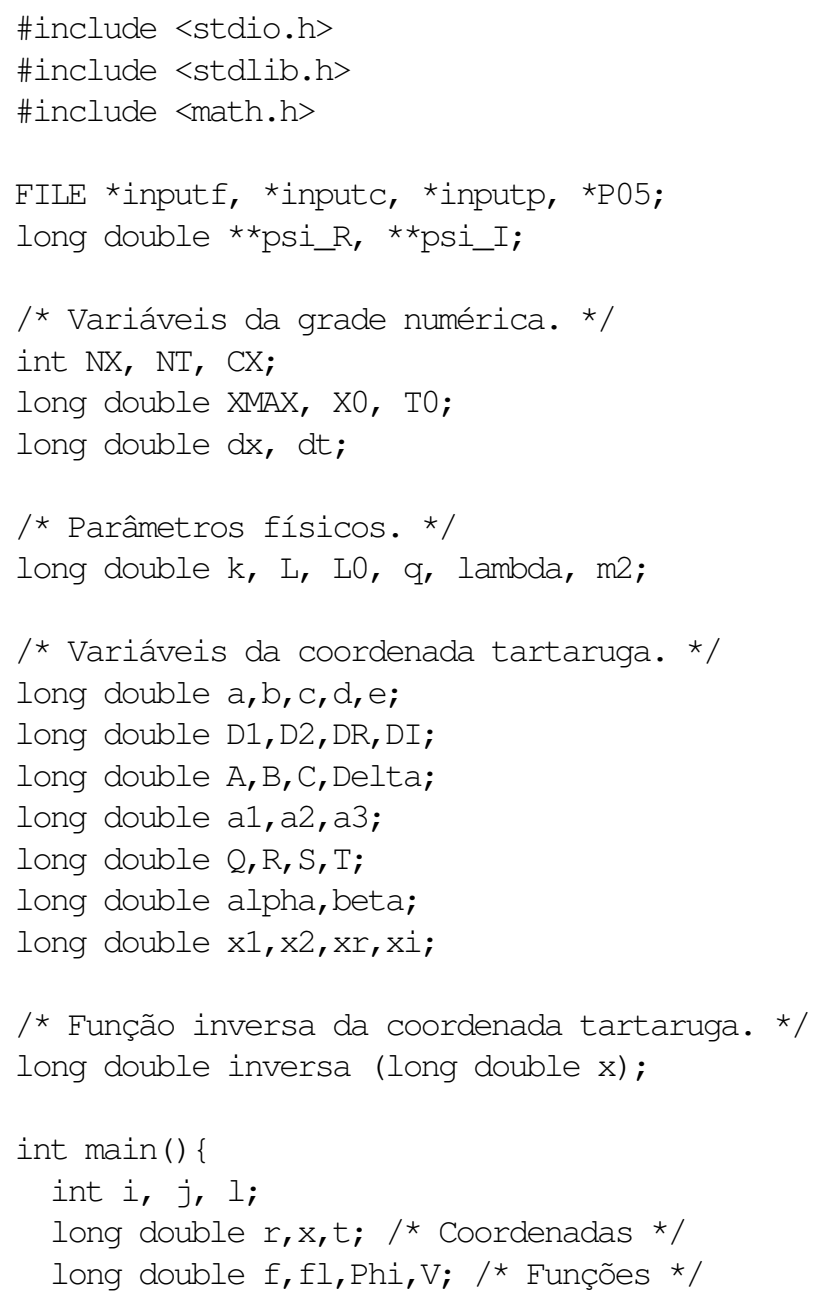




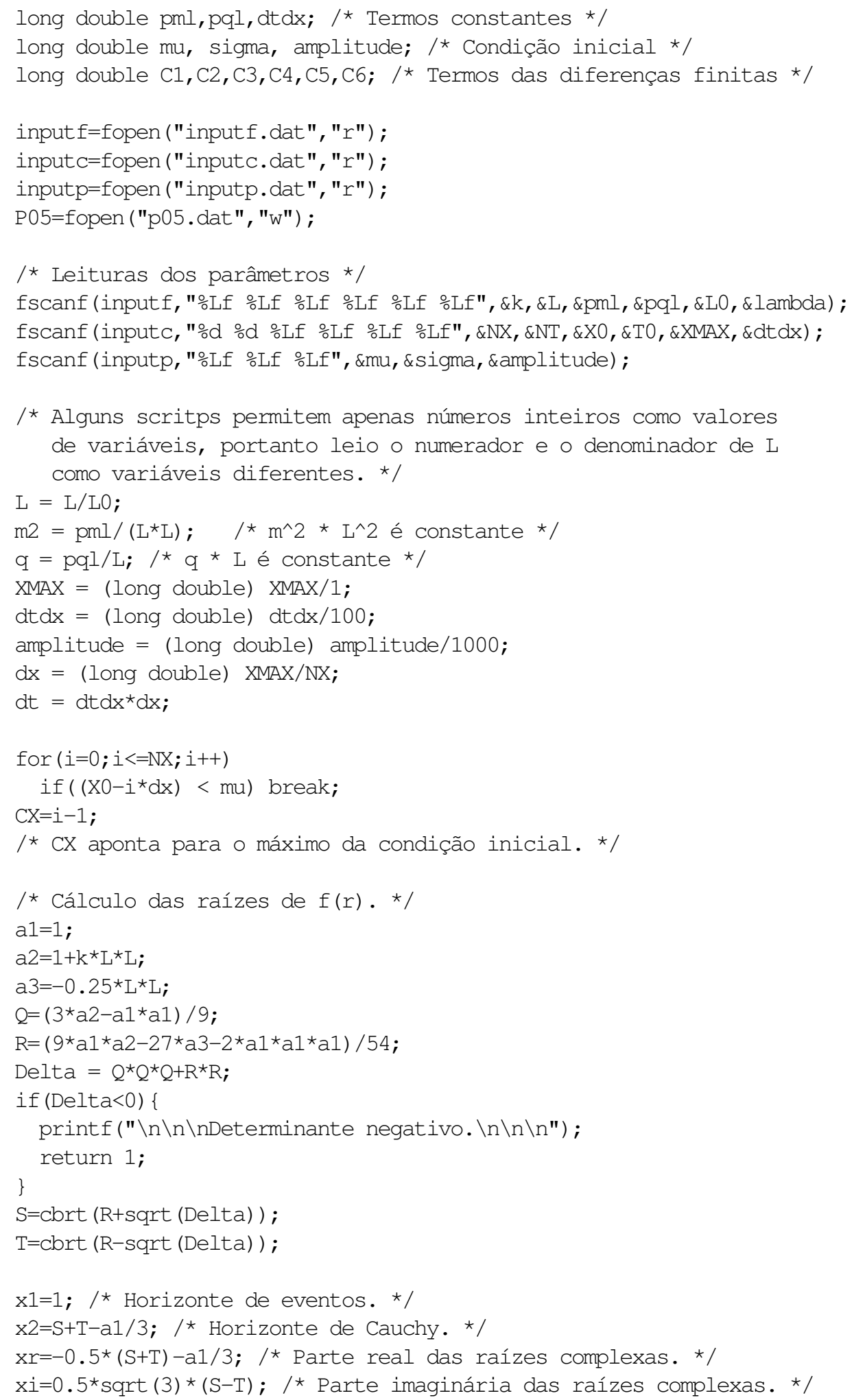




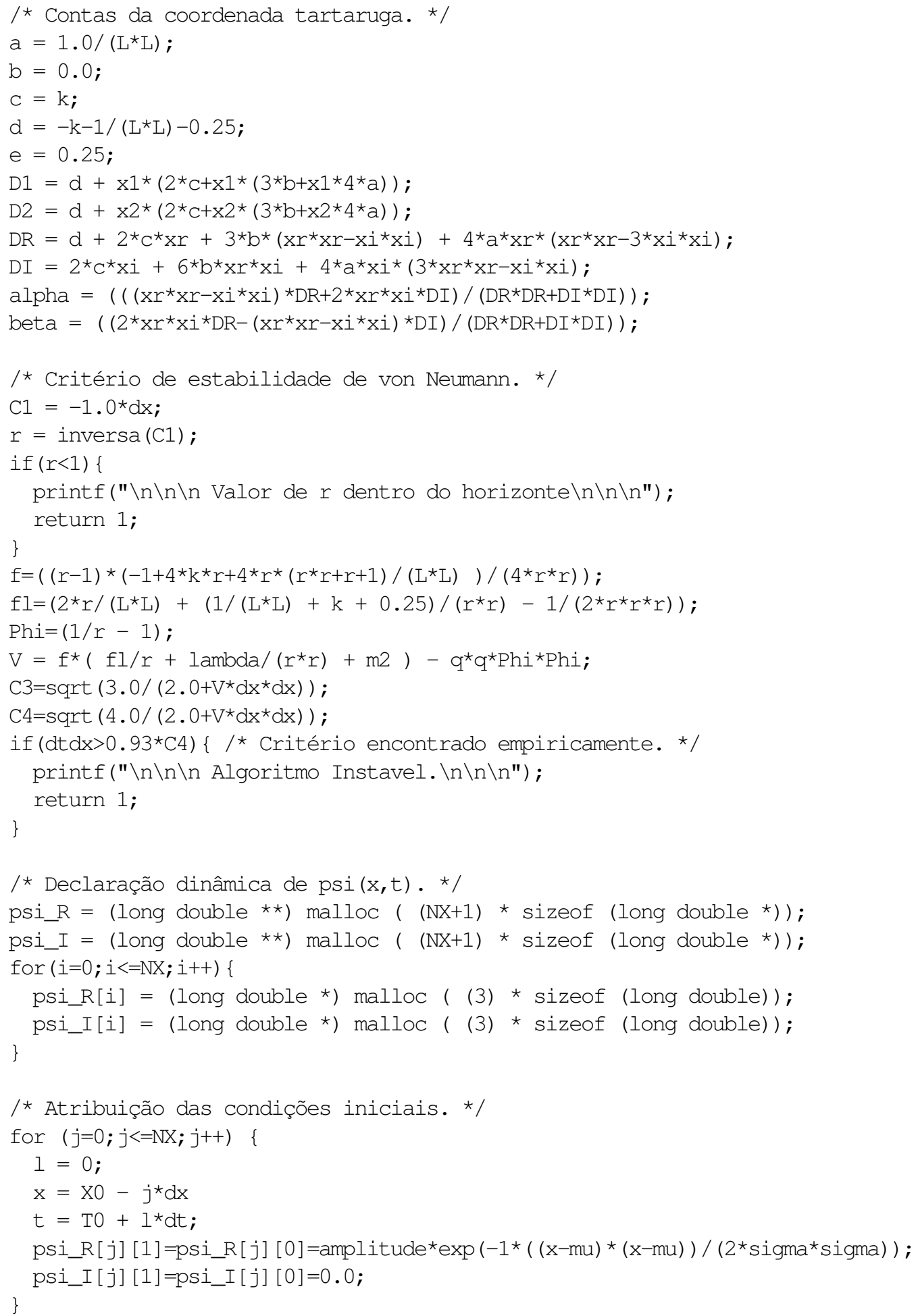




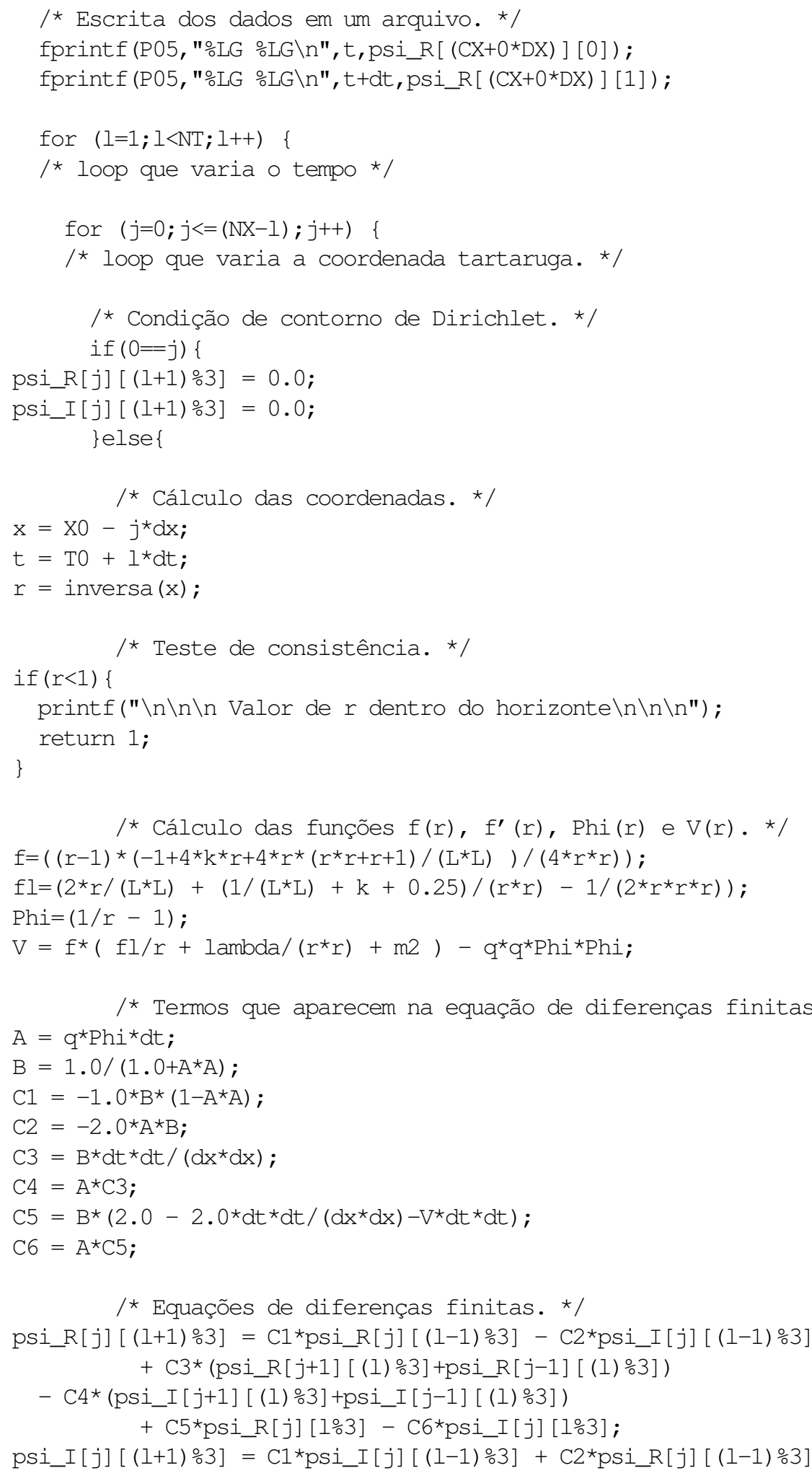




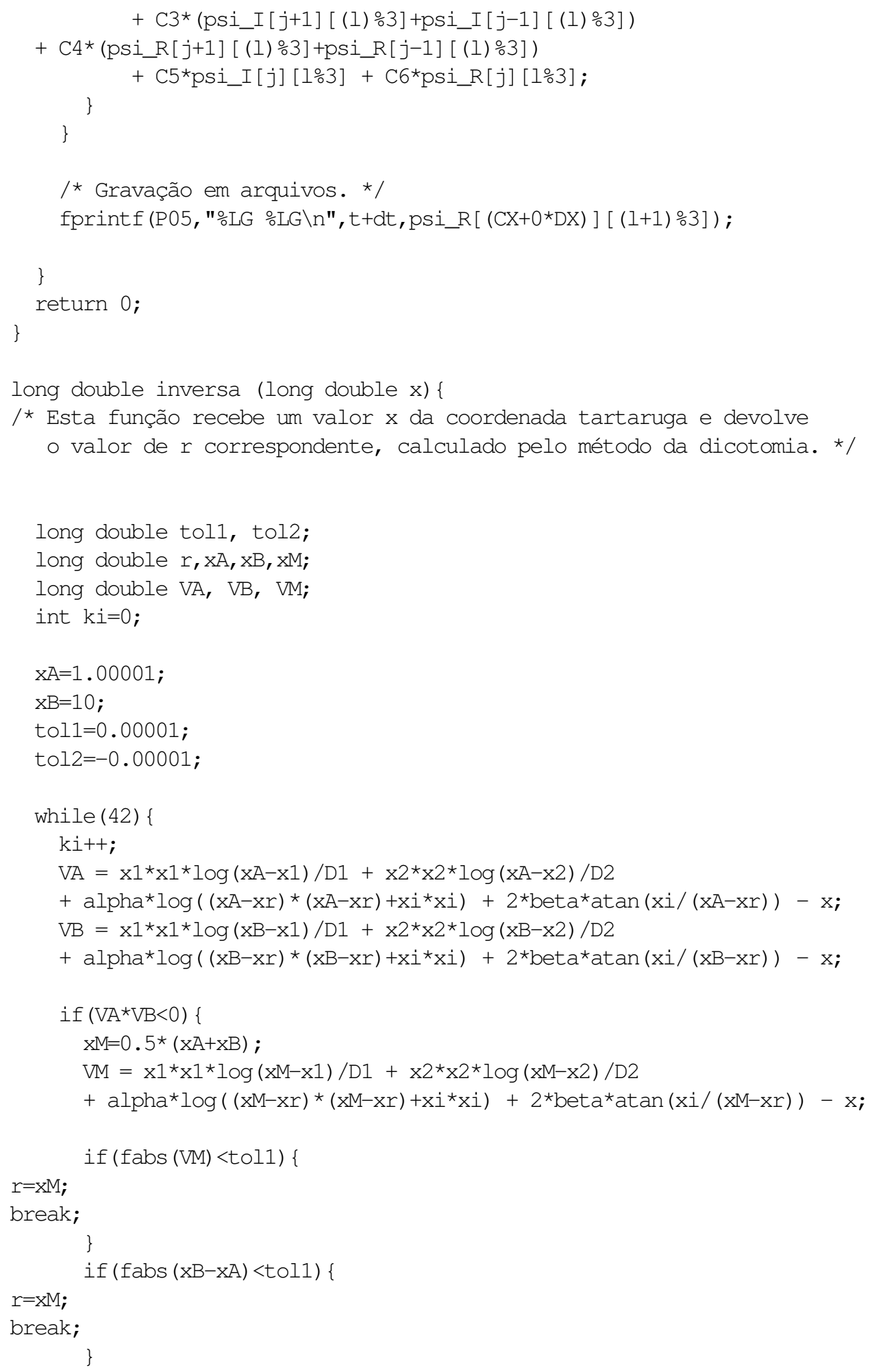




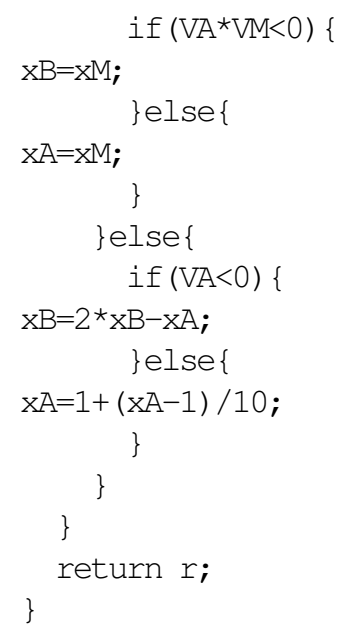




\section{Apêndice C}

\section{Código numérico do método de Horowitz-Hubeny.}

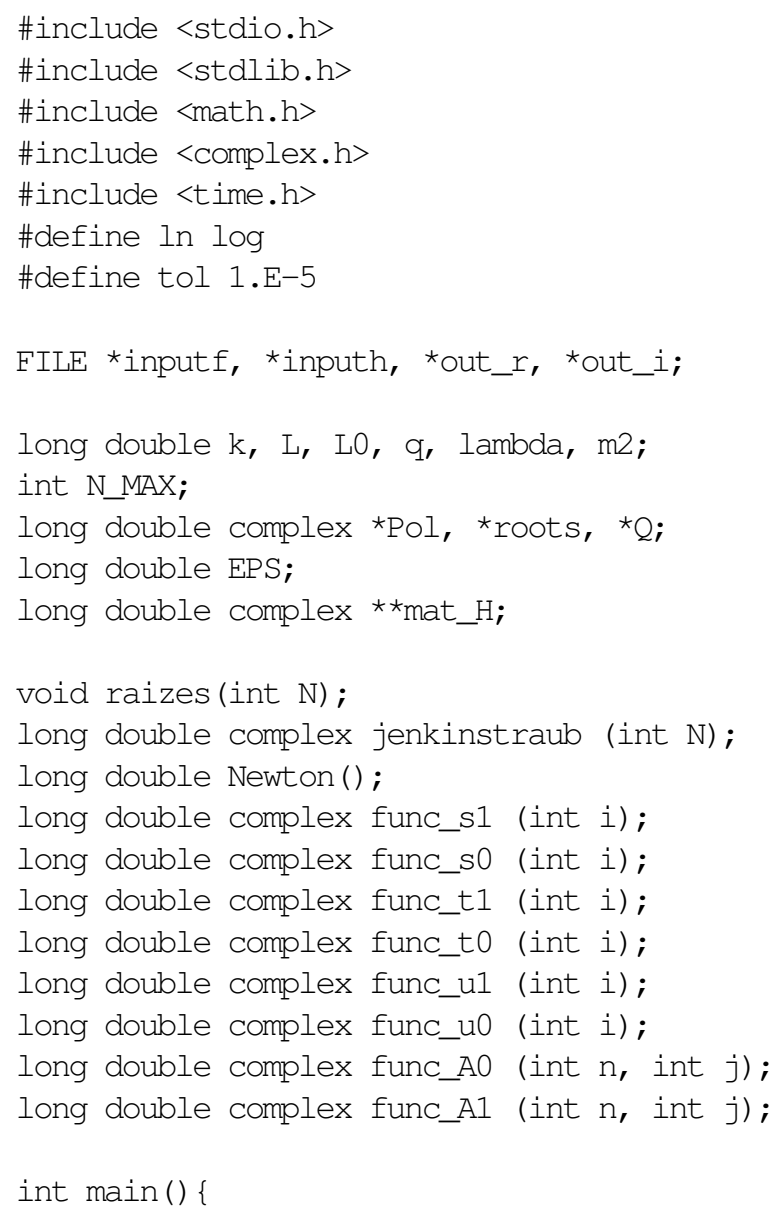




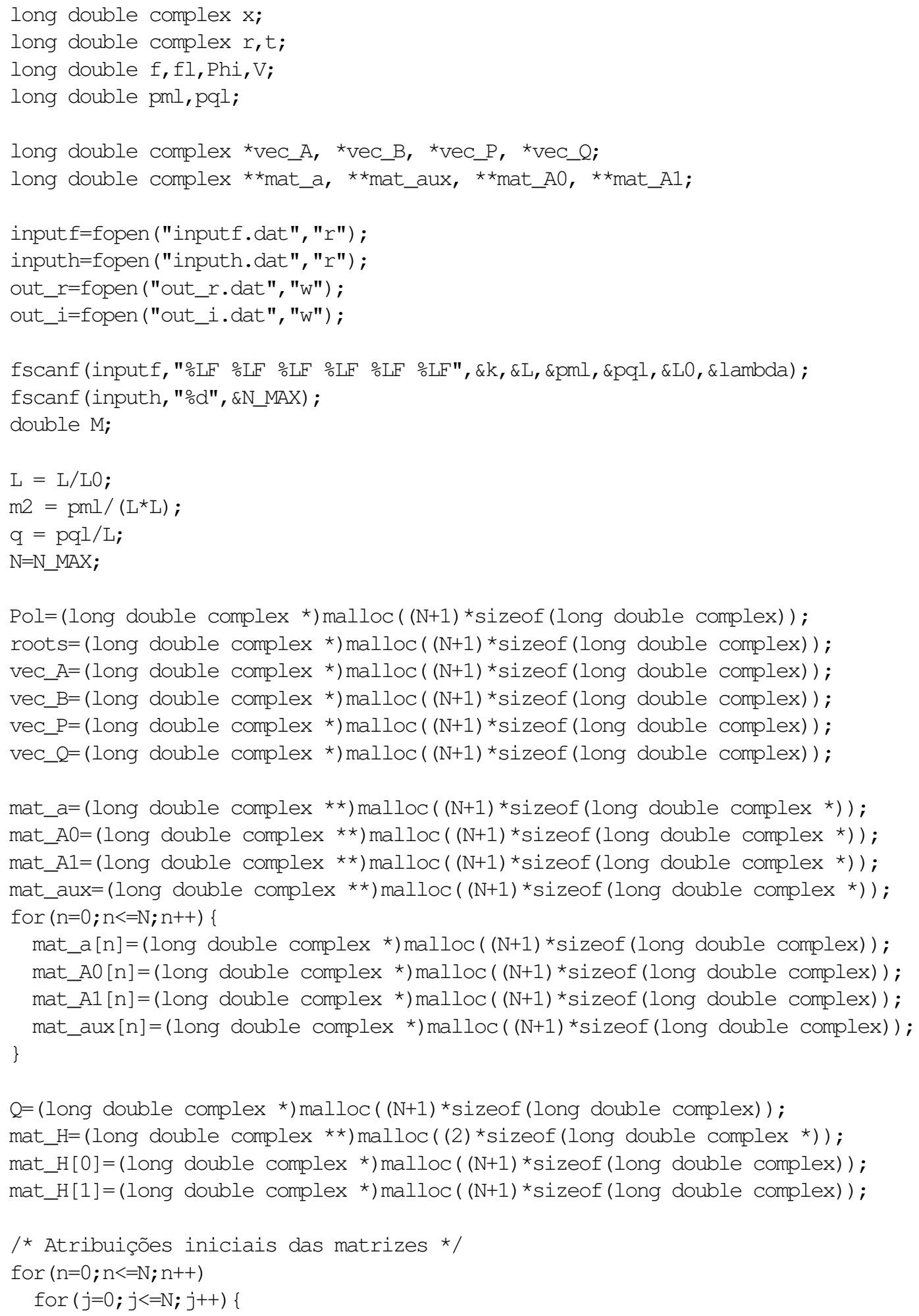




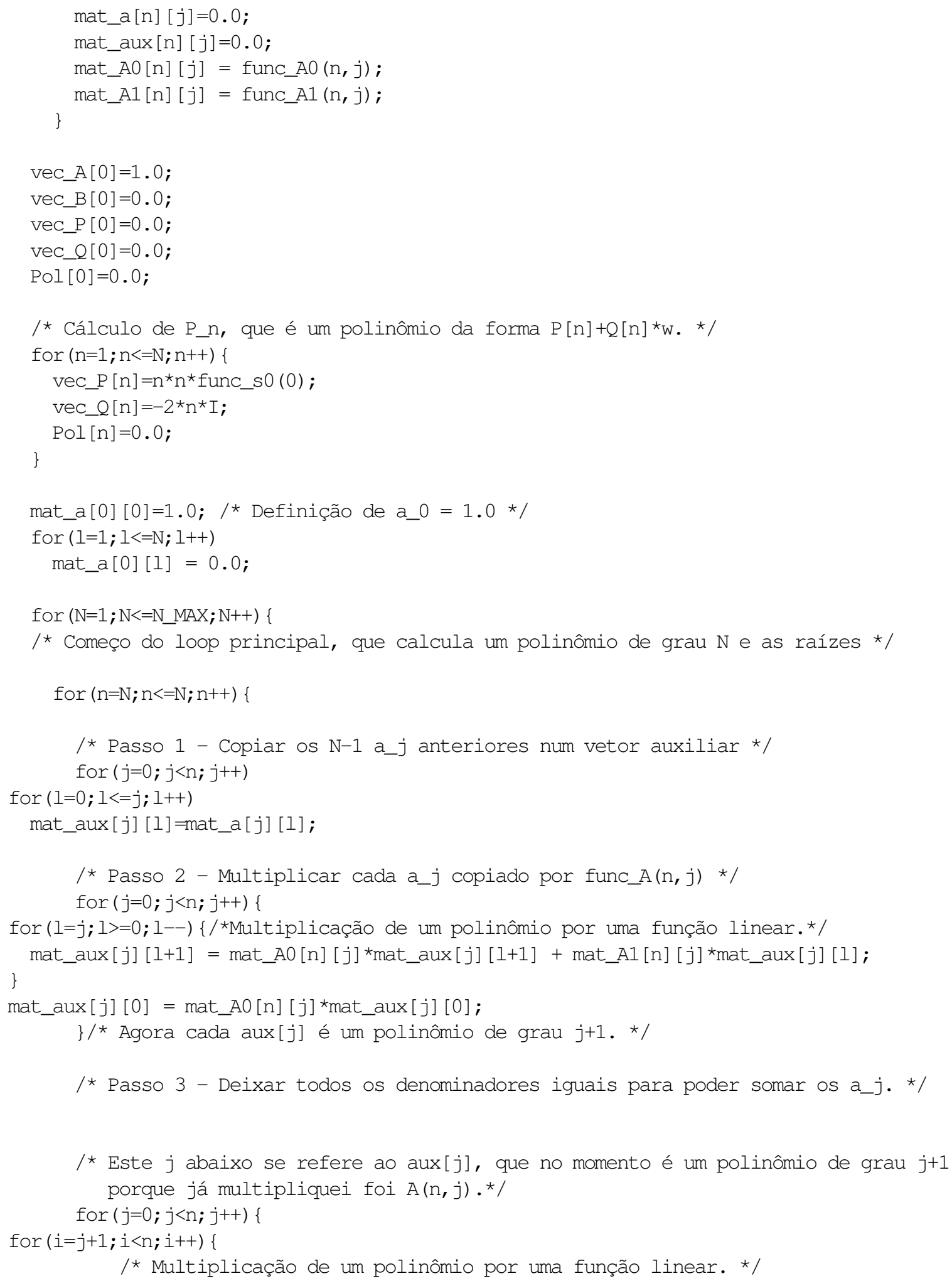




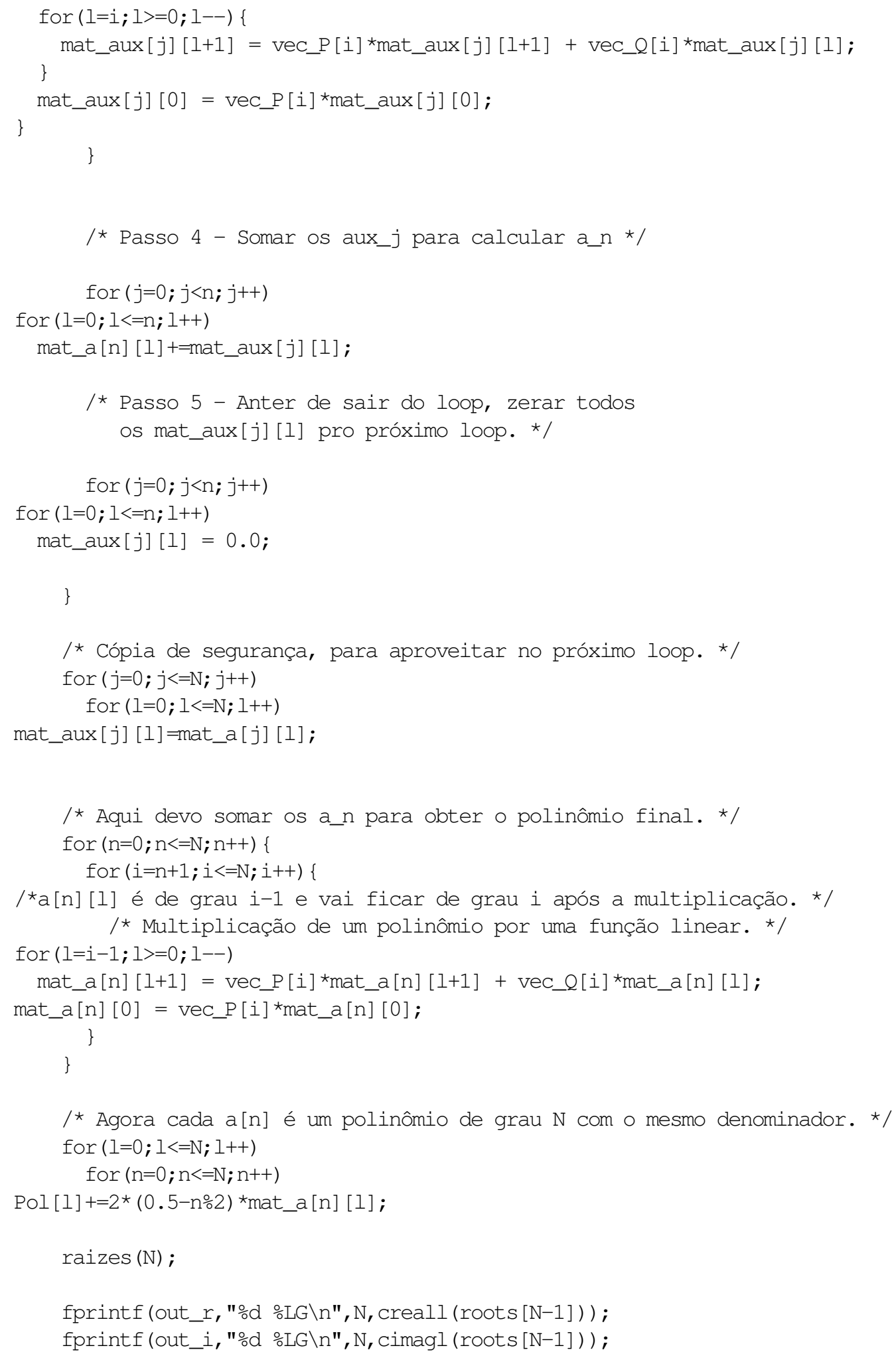




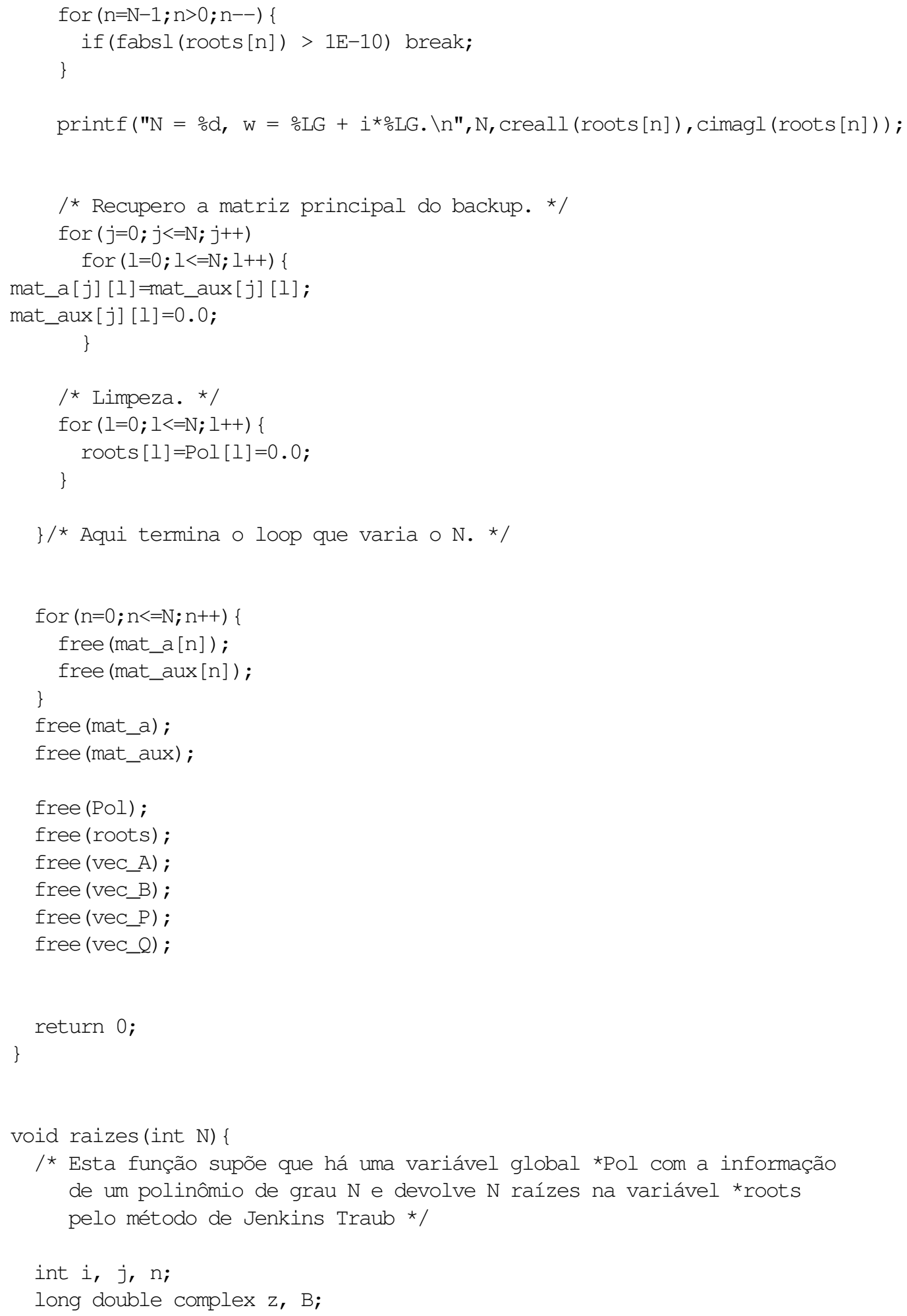




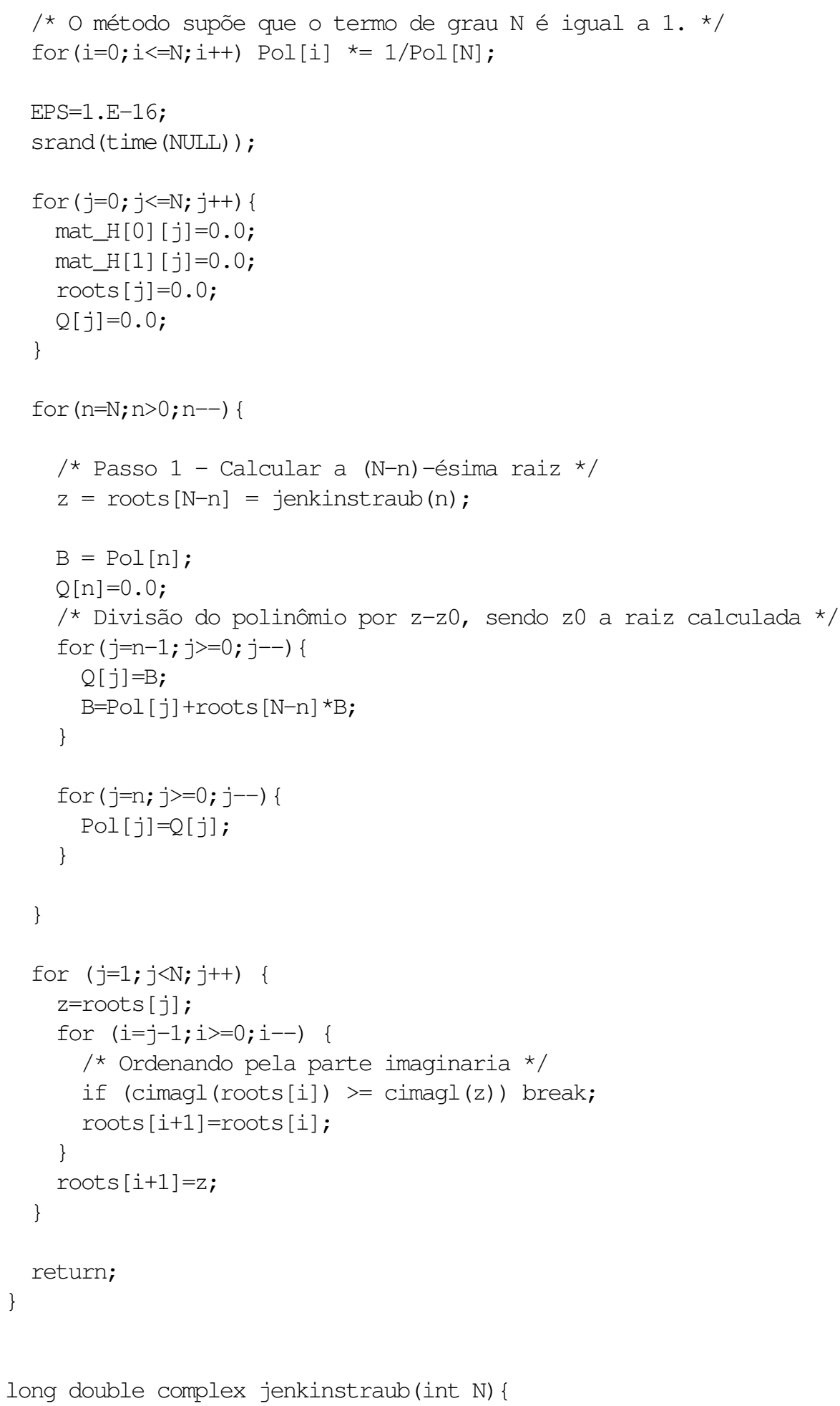




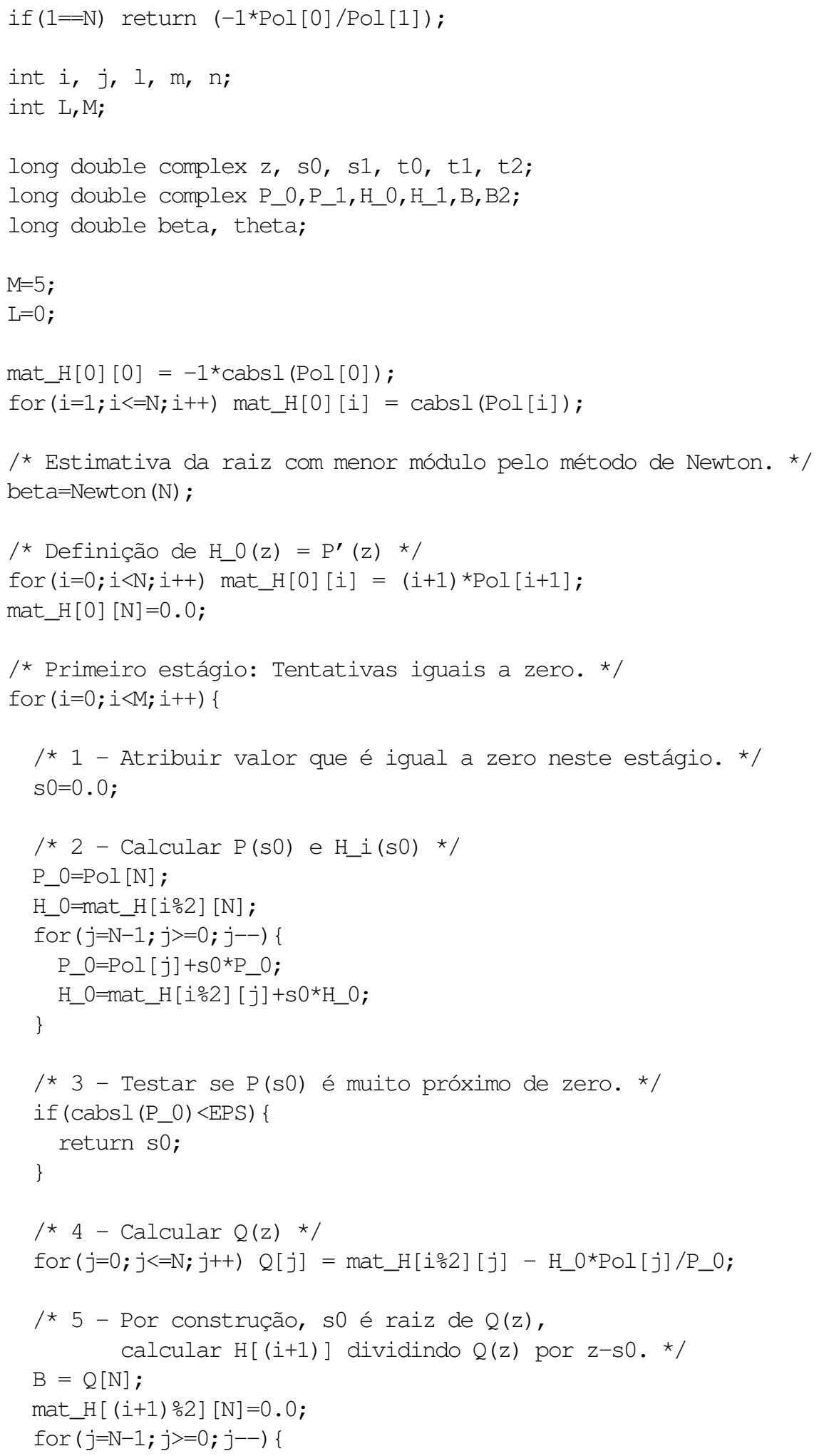




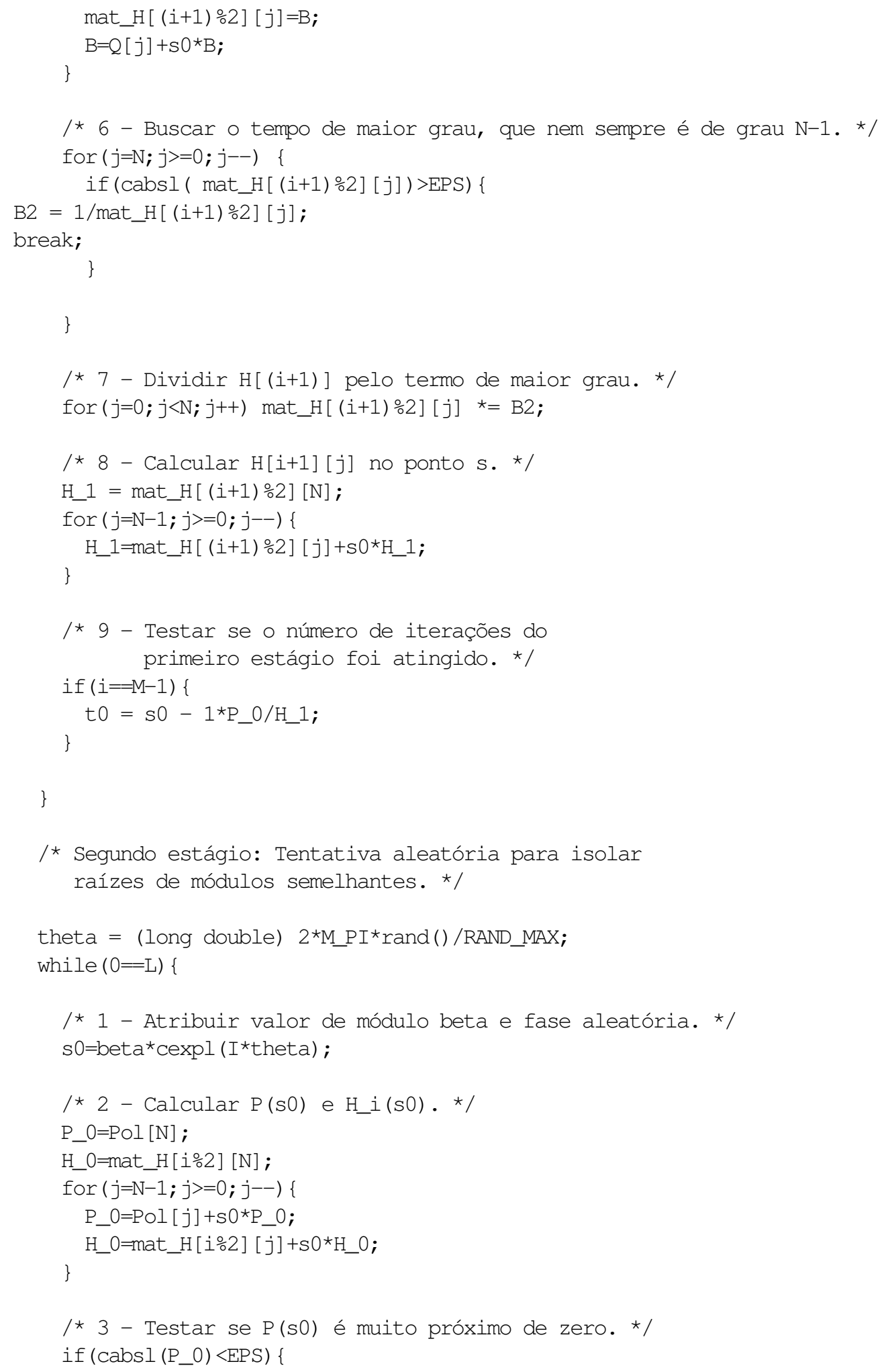




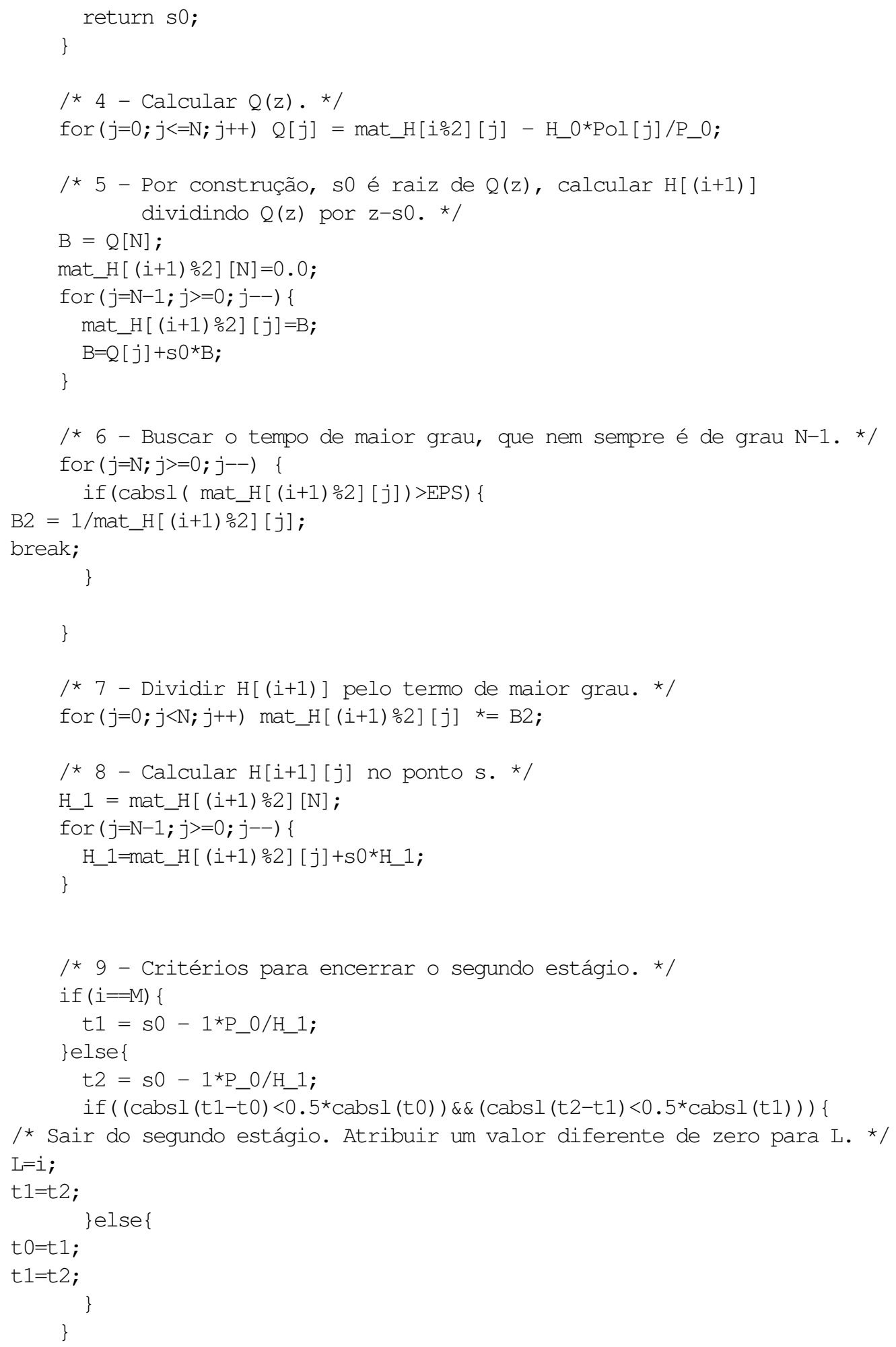




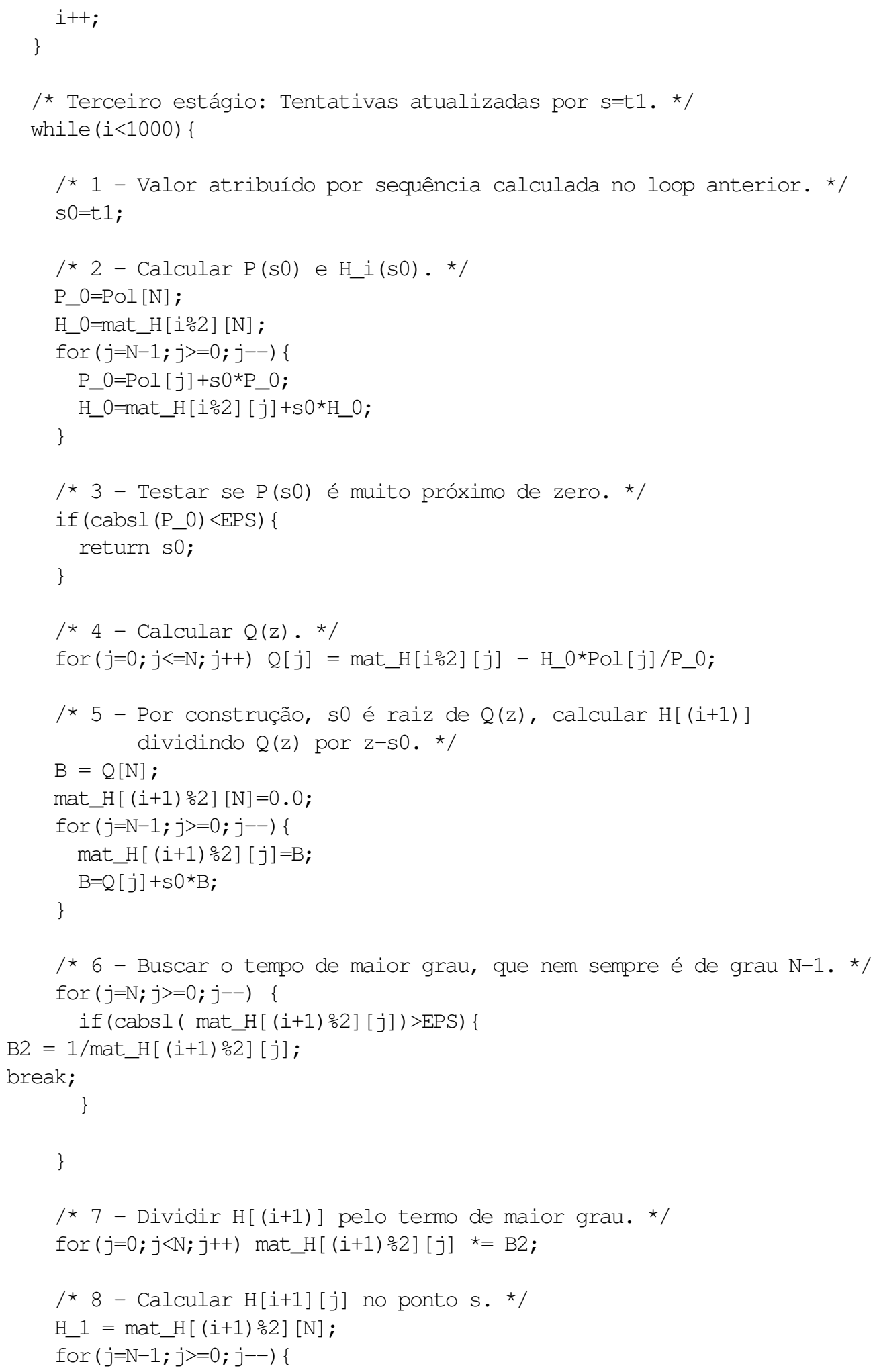




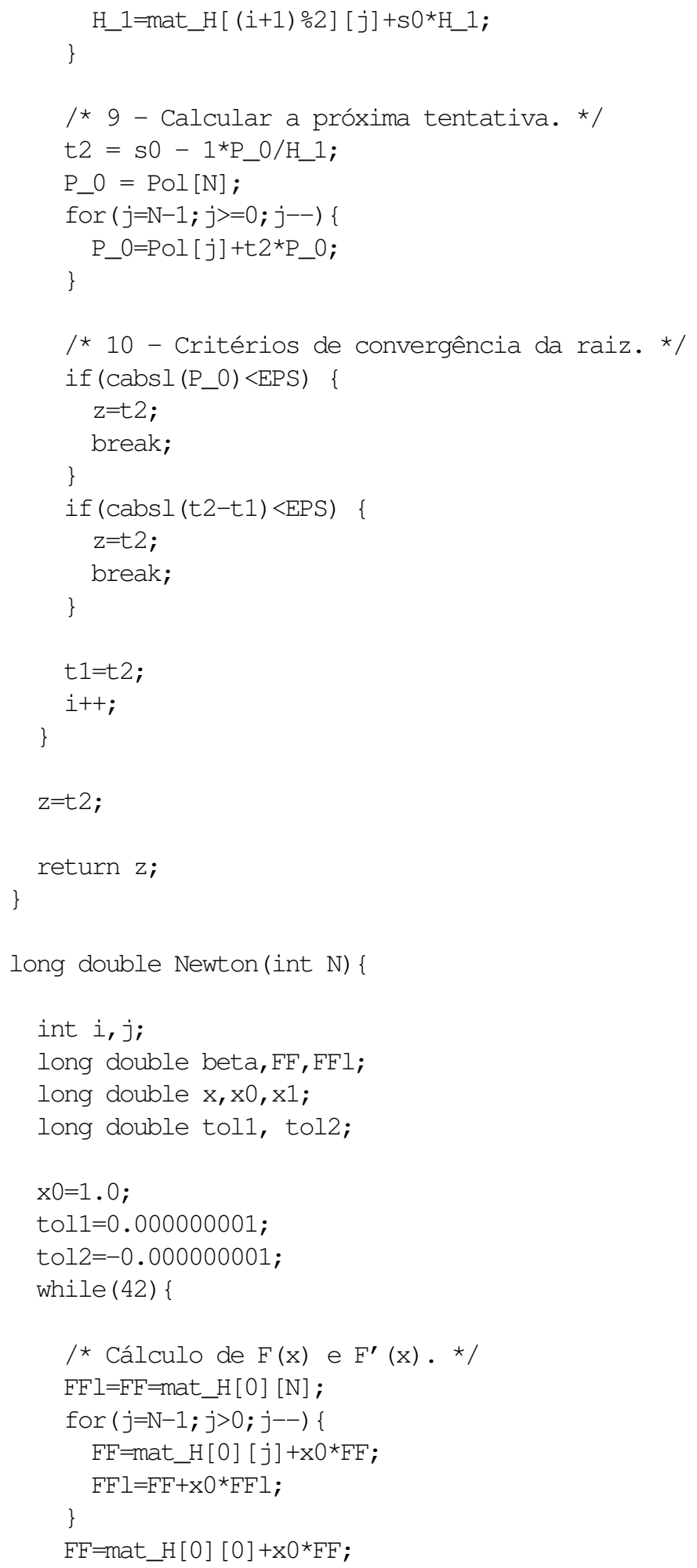




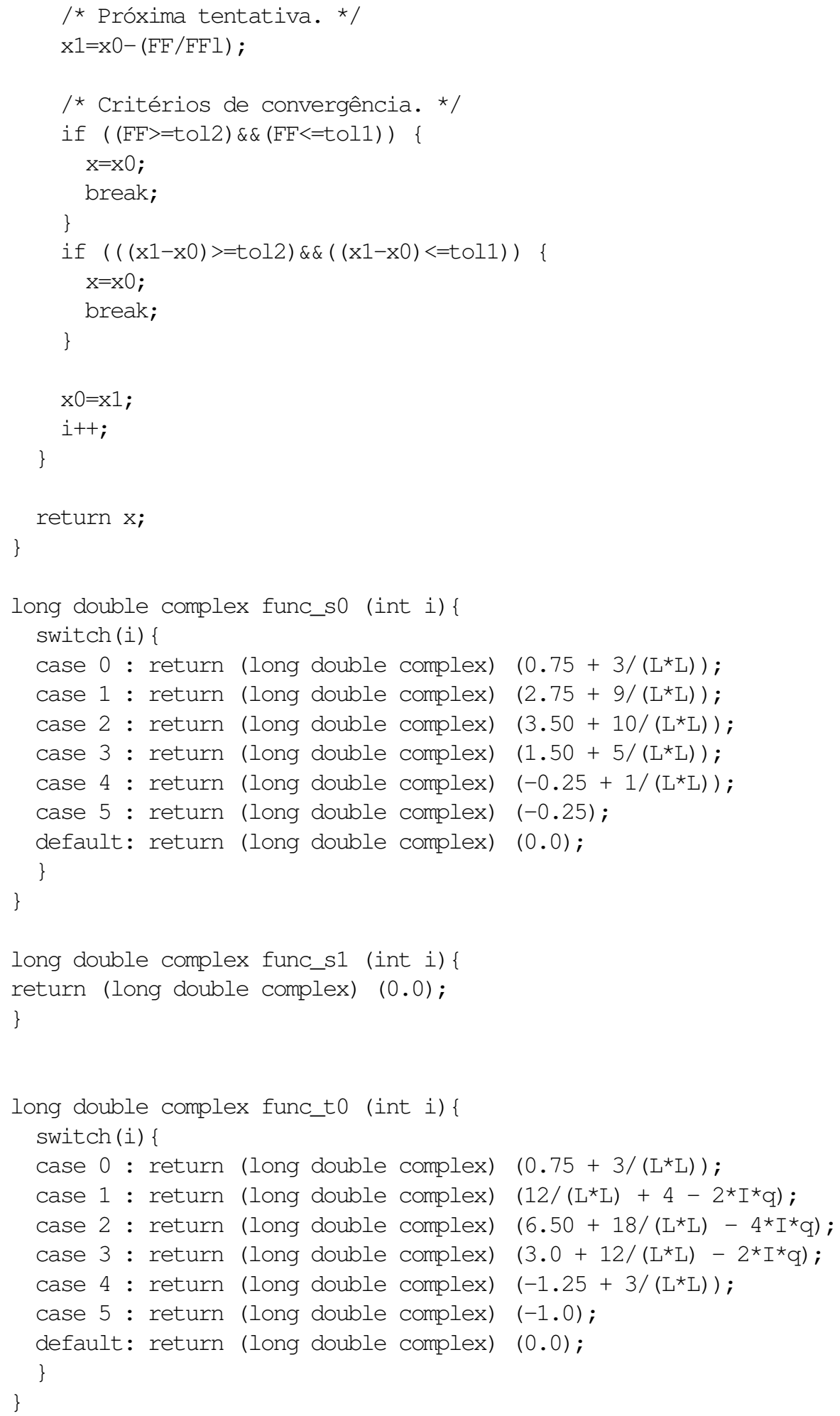




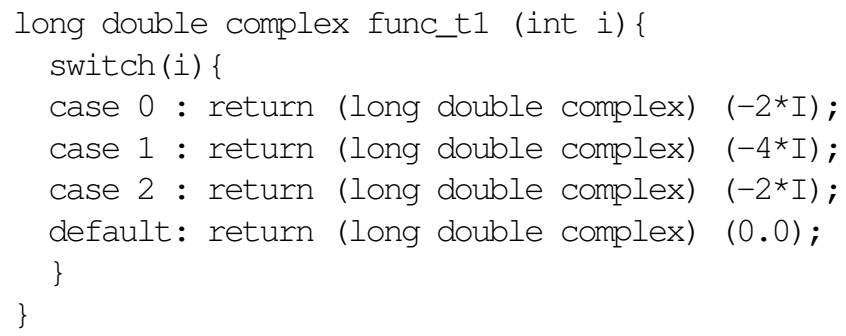





\section{Referências Bibliográficas}

[1] A. Einstein, Die Grundlage der allgemeinen Relativitätstheorie, Annalen Der Physik 49 769-822(1915) . 1

[2] K. Schwarzschild, Über das Gravitationsfeld eines Massenpunktes nach der Einsteinschen Theorie, Sitzungsber. K. Preuss. Akad. Wiss. 1 189-196(1916) . 2

[3] M. D. Kruskal, Maximal Extension of Schwarzschild Metric, Phys. Rev. 119 1743-1745(1960) . 3

[4] S. W. Hawking e G. F. R. Ellis, The Large Scale Structure of Space-Time. Cambridge University Press, Cambridge, 1973. 3

[5] H. Reissner, Über die Eigengravitation des elektrischen Feldes nach der Einsteinschen Theorie, Annalen der Physik 355 106-120(1916) . 3

[6] G. Nordström, Een en ander over de energie van het zwaarte krachtsveld volgens de theorie van Einstein, Verhandl. Koninkl. Ned. Akad.

Wetenschap. 26 1201-1208(1918) . 3

[7] R. d'Inverno, Introducing Einstein's Relativity. Clarendon Press, Oxford, 1992. 3

[8] E. Abdalla, C. E. Pellicer, J. de Oliveira, e A. B. Pavan, Phase transitions and regions of stability in Reissner-Nordström holographic superconductors, Phys. Rev. D 82 124033(2010), arXiv:1010.2806 [hep-th]. 5

[9] H.-P. Nollert, Quasinormal modes: the characteristic 'sound' of black holes and neutron stars, Classical and Quantum Gravity 16 R159(1999) . 7, 51

[10] K. D. Kokkotas e B. Schmidt, Quasi-Normal Modes of Stars and Black Holes, Living Reviews in Relativity 2 (1999) . http://www.livingreviews.org/lrr-1999-2. 8, 51 
[11] T. Regge e J. A. Wheeler, Stability of a Schwarzschild Singularity, Phys. Rev. 108 1063-1069(1957) . 8, 9

[12] F. J. Zerilli, Effective Potential for Even-Parity Regge-Wheeler Gravitational Perturbation Equations, Phys. Rev. Lett. 24 737-738(1970) . 10

[13] C. V. Vishveshwara, Stability of the schwarzschild metric, Phys. Rev. D 1 2870-2879(1970) . 11

[14] R. H. Price, Nonspherical perturbations of relativistic gravitational collapse. 1. Scalar and gravitational perturbations, Phys. Rev. D 5 2419-2438(1972) . 11

[15] R. M. Wald, Note on the stability of the Schwarzschild metric, J. Math. Phys. 20 1056(1979). 11, 12

[16] G. T. Horowitz e V. E. Hubeny, Quasinormal modes of AdS black holes and the approach to thermal equilibrium, Phys. Rev. D 62 024027(2000), arXiv:hep-th/9909056. 12, 15, 26, 41, 43

[17] B. F. Schutz e C. M. Will, Black hole normal modes - A semianalytic approach, Astrophys. J. 291 L33-L36(1985) . 14

[18] S. Iyer e C. M. Will, Black-hole normal modes: A WKB approach. I. Foundations and application of a higher-order WKB analysis of potential-barrier scattering, Phys. Rev. D 35 3621-3631(1987) . 15

[19] S. Iyer, Black-hole normal modes: A WKB approach. II. Schwarzschild black holes, Phys. Rev. D 35 3632-3636(1987) . 15

[20] K. D. Kokkotas e B. F. Schutz, Black-hole normal modes: A WKB approach. III. The Reissner-Nordström black hole, Phys. Rev. D 37 3378-3387(1988) . 15

[21] E. Seidel e S. Iyer, Black-hole normal modes: A WKB approach. IV. Kerr black holes, Phys. Rev. D 41 374-382(1990) . 15

[22] P. R. Garabedian, Partial Differential Equations. American Mathematical Society, Providence, Rhode Island, 2007. 17, 76

[23] R. Courant e D. Hilbert, Methods of Mathematical Physics, volume 2. Wiley-VCH, 1962. 17, 76 
[24] H. Nastase, Introduction to AdS-CFT, ArXiv e-prints (2007), arXiv:0712.0689 [hep-th]. 19

[25] O. Aharony, S. S. Gubser, J. Maldacena, H. Ooguri, e Y. Oz, Large N field theories, string theory and gravity, Physics Reports 323183 - 386(2000), arXiv:hep-th/9905111. 19, 20, 21

[26] G. T. Horowitz, Introduction to Holographic Superconductors, ArXiv e-prints (2010), arXiv:1002.1722 [hep-th]. 19, 23, 25

[27] D. Bigatti e L. Susskind, TASI lectures on the Holographic Principle, em Strings, Branes and Gravity TASI 99, J. A. Harvey, S. Kachru, \& E. Silverstein, editor, pp. 883-933. Outubro, 2001. arXiv:hep-th/0002044. 19, 21

[28] G. 't Hooft, A planar diagram theory for strong interactions, Nuclear Physics B 72461 - 473(1974). 19

[29] J. Polchinski, Dirichlet Branes and Ramond-Ramond Charges, Phys. Rev. Lett. 75 4724-4727(1995) . 19

[30] J. Maldacena, The Large-N Limit of Superconformal Field Theories and Supergravity, International Journal of Theoretical Physics 38 1113-1133(1999), arXiv:hep-th/9711200. 20, 21

[31] E. Witten, Anti De Sitter Space And Holography, Adv.Theor.Math.Phys. 2 253-291(1998), arXiv:hep-th/9802150. 20

[32] G. 't Hooft, Dimensional Reduction in Quantum Gravity, ArXiv General Relativity and Quantum Cosmology e-prints (1993), arXiv:gr-qC/9310026. 20

[33] L. Susskind, The world as a hologram, Journal of Mathematical Physics 36 6377-6396(1995), arXiv:hep-th/9409089. 20

[34] S. A. Hartnoll, C. P. Herzog, e G. T. Horowitz, Holographic superconductors, Journal of High Energy Physics 2008 015(2008), arXiv:0810.1563v1 [hep-th]. 23

[35] R. Gregory, S. Kanno, e J. Soda, Holographic superconductors with higher curvature corrections, Journal of High Energy Physics 10 10-+(2009), arXiv:0907.3203 [hep-th]. 24 
[36] Q. Pan, B. Wang, E. Papantonopoulos, J. de Oliveira, e A. B. Pavan, Holographic superconductors with various condensates in Einstein-Gauss-Bonnet gravity, Phys. Rev. D 81 106007(2010), arXiv:0912.2475 [hep-th]. 25

[37] S. A. Hartnoll e P. K. Kovtun, Hall conductivity from dyonic black holes, Phys. Rev. D 76 066001(2007), arXiv:0704.1160 [hep-th]. 25

[38] D. Birmingham, I. Sachs, e S. N. Solodukhin, Conformal Field Theory Interpretation of Black Hole Quasinormal Modes, Phys. Rev. Lett. 88 151301(2002), arXiv:hep-th/0112055. 26

[39] M. Bañados, C. Teitelboim, e J. Zanelli, Black hole in three-dimensional spacetime, Phys. Rev. Lett. 69 1849-1851(1992), arXiv:hep-th/9204099. 26

[40] S. S. Gubser, Breaking an Abelian gauge symmetry near a black hole horizon, Phys. Rev. D 78 065034(2008), arXiv:0801.2977 [hep-th]. $29,30,31,32,33,35,40,42,51$

[41] S. S. Gubser, Phase transitions near black hole horizons, Classical and Quantum Gravity 22 5121(2005), arXiv:hep-th/0505189v2. 29

[42] A. Bachelot e A. Motet-Bachelot, Les résonances d'un trou noir de Schwarzschild., Ann. Inst. Henri Poincaré, Phys. Théor., Vol. 59, No. 1, p. 3 - $68593-68(1993)$. 40

[43] B. Cuadros-Melgar e C. E. Pellicer, Instability of the time dependent Hořava-Witten model, Phys. Rev. D 77 104030(2008), arXiv:0712.2155 [hep-th]. 52

[44] B. Cuadros-Melgar, J. de Oliveira, e C. E. Pellicer, Stability Analysis and Area Spectrum of 3-Dimensional Lifshitz Black Holes, ArXiv e-prints (2011), arXiv:1110.4856 [hep-th]. 52

[45] E. A. Bergshoeff, O. Hohm, e P. K. Townsend, Massive Gravity in Three Dimensions, Phys. Rev. Lett. 102 201301(2009), arXiv:0901.1766 [hep-th]. 52

[46] C. E. Pellicer, E. G. M. Ferreira, D. C. Guariento, A. A. Costa, L. L. Graef, A. Coelho, e E. Abdalla, The role of Dark Matter interaction in galaxy clusters, ArXiv e-prints (2011), arXiv:1102.5113 [astro-ph.CO]. 52 
[47] J. Lee, The Misalignments between Matter and Galaxy Distributions in Triaxial Clusters: A Signature of a Possible Fifth Force?, ArXiv e-prints (2010), arXiv:1008.4620 [astro-ph.Co]. 52

[48] M. R. Hestenes e E. Stiefel, Methods of Conjugate Gradients for Solving Linear Systems, Journal Of Research Of The National Bureau Of Standards 49 409-436(1952) . 56

[49] M. Jenkins e J. Traub, A three-stage variable-shift iteration for polynomial zeros and its relation to generalized rayleigh iteration, Numerische Mathematik 14 252-263(1970). 59

[50] A. Ralston e P. Rabinowitz, A First Course in Numerical Analysis. Dover Publications, Inc., Mineola,New York, 2 edição, 2001. 61, 62, 64, 71

[51] J. H. Wilkinson, The evaluation of the zeros of ill-conditioned polynomials. Part I, Numerische Mathematik 1 150-166(1959) . 63

[52] C. Runge, Über die numerische Auflösung von Differentialgleichungen, Mathematische Annalen 46 167-178(1895) . 68

[53] W. Kutta, Beitrag zur näherungweisen Integration totaler Differentialgleichungen, Zeit. Math. Physik 46 435-453(1901) . 68 